\title{
Fourth post-Newtonian effective-one-body Hamiltonians with generic spins
}

\author{
Mohammed Khalilø, ${ }^{1,2, *}$ Jan Steinhoff $\odot,{ }^{1, \dagger}$ Justin Vines $\oplus^{1, \$}$ and Alessandra Buonanno ${ }^{1,2, \S}$ \\ ${ }^{1}$ Max Planck Institute for Gravitational Physics (Albert Einstein Institute), \\ Am Mühlenberg 1, Potsdam 14476, Germany \\ ${ }^{2}$ Department of Physics, University of Maryland, College Park, Maryland 20742, USA
}

(Received 15 March 2020; accepted 30 April 2020; published 18 May 2020)

\begin{abstract}
In a compact binary coalescence, the spins of the compact objects can have a significant effect on the orbital motion and gravitational-wave (GW) emission. For generic spin orientations, the orbital plane precesses, leading to characteristic modulations of the GW signal. The observation of precession effects is crucial to discriminate among different binary formation scenarios and to carry out precise tests of general relativity. Here, we work toward an improved description of spin effects in binary inspirals, within the effective-one-body (EOB) formalism, which is commonly used to build waveform models for LIGO and Virgo data analysis. We derive EOB Hamiltonians including the complete fourth post-Newtonian (4PN) conservative dynamics, which is the current state of the art. We place no restrictions on the spin orientations or magnitudes, or on the type of compact object (e.g., black hole or neutron star), and we produce the first generic-spin EOB Hamiltonians complete at 4PN order. We consider multiple spinning EOB Hamiltonians, which are more or less direct extensions of the varieties found in previous literature, and we suggest another simplified variant. Finally, we compare the circular-orbit, aligned-spin binding-energy functions derived from the EOB Hamiltonians to numerical-relativity simulations of the late inspiral. While finding that all proposed Hamiltonians perform reasonably well, we point out some interesting differences, which could guide the selection of a simpler, and thus faster-to-evolve EOB Hamiltonian to be used in future LIGO and Virgo inference studies.
\end{abstract}

DOI: 10.1103/PhysRevD.101.104034

\section{INTRODUCTION}

The observation of gravitational waves (GWs) from coalescing binaries [1-4] using a continually improving network of GW detectors [5-8] is a milestone in fundamental physics and astrophysics. As the detectors increase their sensitivity, we will observe more events, with larger signal-to-noise ratios, spanning a larger region of the parameter space. Thus, to faithfully recover the sources' properties, it is important to improve the accuracy of models of waveforms from binaries of compact objects (black holes and/or neutron stars) on generic orbits and with generic spin orientations. In the generic case, the orbital plane and the objects' spins precess about the direction of the system's total angular momentum, leading

*mohammed.khalil@aei.mpg.de

†jan.steinhoff@aei.mpg.de

justin.vines@aei.mpg.de

§alessandra.buonanno@aei.mpg.de

Published by the American Physical Society under the terms of the Creative Commons Attribution 4.0 International license. Further distribution of this work must maintain attribution to the author(s) and the published article's title, journal citation, and DOI. Open access publication funded by the Max Planck Society. to modulations of the GW signal which are a smoking-gun signature of the dynamical influence of the spins. Including such precession effects in GW template models, as opposed to restricting to the simpler aligned-spin case with no precession, is important for more inclusive GW searches, more accurate inference studies, and tests of general relativity.

The effective-one-body (EOB) framework $[9,10]$ aims at providing a synergy between multiple analytical approximations and numerical-relativity (NR) simulations of relativistic inspiraling binaries. The core ingredient of the EOB approach is the EOB Hamiltonian, a canonical Hamiltonian describing the binary's (conservative) orbital dynamics, which both (i) agrees, in its post-Newtonian (PN) expansion, ${ }^{1}$ with known results for arbitrary mass ratios from PN calculations (in the weak-field and lowspeed regime), and (ii) becomes, in the extreme-mass-ratio limit, an exact Hamiltonian for a test (or probe) particle in an exact black-hole spacetime, valid for arbitrary separations and speeds. The EOB Hamiltonian is naturally expressed as a deformation of the zero-mass-ratio testparticle Hamiltonian, with the deformation determined by

\footnotetext{
${ }^{1}$ Recently, also the post-Minkowskian (weak-field) approximation for unbound orbits is considered; see below.
} 
TABLE I. The SEOB Hamiltonians used in this paper and the differences between them. All Hamiltonians include complete 4PN results for generic spins and compact objects, except for the last Hamiltonian, which is for aligned spins.

\begin{tabular}{llr}
\hline \hline $\mathrm{SEOB}$ & \multicolumn{1}{c}{ Definition } & References \\
\hline $\mathrm{SEOB}_{\mathrm{TS}}$ & Based on the Hamiltonian for a test spin (TS) in Kerr spacetime & {$[27,28]$} \\
$\mathrm{SEOB}_{\mathrm{TM}}^{r_{c}}$ & Based on the Hamiltonian for a test mass (TM) in Kerr spacetime; & {$[17]$} \\
$\mathrm{SEOB}_{\mathrm{TM}}$ & it uses the centrifugal radius $r_{c}$ & This paper \\
$\mathrm{SEOB}_{\mathrm{TM}}^{r_{c}, \text { align }}$ & Simplified version of SEOB & $r_{\mathrm{TM}}^{r_{c}} ;$ it does not use $r_{c} ;$ \\
& it uses different factorization for spin corrections & {$[19,23,47]$} \\
\hline \hline
\end{tabular}

finite-mass-ratio results from the PN approximation. For example, the original (nonspinning) EOB Hamiltonian [9] becomes, as the mass ratio goes to zero, the exact Hamiltonian for a test mass undergoing geodesic motion in a Schwarzschild (nonspinning black hole) spacetime.

In generalizing to spinning black holes, the first natural replacement for the Schwarzschild-geodesic Hamiltonian is the Hamiltonian for geodesic (test-mass) motion in an exact Kerr (spinning black hole) spacetime. ${ }^{2}$ A spinning EOB (SEOB) Hamiltonian incorporating the Kerr-geodesic limit was first constructed in Ref. [12] including leading-order (LO) spin-orbit and LO spin-squared effects in the PN expansion. This was later extended to the next-to-leading (NLO) [13] and next-to-NLO (NNLO) [14] spin-orbit levels, and to the NLO spin-squared level for aligned spins $[15,16]$ and then for generic (precessing) spins [17]. The Kerr-geodesic-based approach for aligned spins has been further developed in Refs. [18-24], e.g., by including matter effects (for neutron stars) and calibration to NR simulations. A second category of SEOB Hamiltonians is based on the Hamiltonian for a spinning test body (test spin) in a Kerr background [25,26], first developed with NLO [27] and then NNLO [28] spin-orbit terms and with LO spin-squared terms. Such Hamiltonians have always been applicable for generic (precessing) spins. They have been generalized to include tidal effects in Refs. [29,30], they have been used for studies of extreme-mass-ratio binaries in Ref. [31] and periastron advance in Ref. [32], and they have been refined and calibrated to NR simulations in Refs. [33-38]. EOB Hamiltonians have also been constructed to include information from gravitational self-force calculations [39-42] (for extreme mass ratios) and from the post-Minkowskian approximation [43-45] (assuming weak fields but allowing arbitrary speeds). A recent comparison of various SEOB waveform models is given in Ref. [46]. Waveform models constructed with the SEOB Hamiltonians based on a spinning test body in a Kerr

\footnotetext{
${ }^{2}$ With the aim of building a first inspiral-merger-ringdown waveform model for generic spins, Ref. [11] employed a spinning EOB Hamiltonian built by adding to the Schwarzschild-geodesic EOB Hamiltonian the PN-expanded spin Hamiltonian.
}

background [34-38] have been employed in template banks of LIGO and Virgo, and inference studies of binary black holes $[1,2,4]$. For parameter estimation of binary neutron stars, both classes of SEOB Hamiltonians have been employed in Ref. [4].

The goal of the present paper is to construct SEOB Hamiltonians for compact binaries (black holes or neutron stars) that include all known PN results to 4PN order for generic orbits and spin orientations. Beyond the up-toNNLO spin-orbit and spin-squared contributions, the 4PN level includes also the LO cubic and quartic in spin terms. Previous work is not complete to 4PN order for generic spins $[17,27,28]$ or complete to $4 \mathrm{PN}$ but valid for aligned spins only $[19,22,23,47]$. We construct three SEOB Hamiltonians in this paper: (i) a Hamiltonian based on Ref. [17], which uses the idea of "centrifugal radius" $r_{c}$ [19], while recovering the Kerr-geodesic limit; (ii) a simplified version of the Hamiltonian from Ref. [17] that does not use a centrifugal radius and has a different factorization for the PN spin corrections, similarly recovering the Kerr-geodesic limit; and (iii) one Hamiltonian following Refs. $[25,27,28]$ which recovers the dynamics of a spinning test body in the Kerr spacetime in the small-mass-ratio limit (see Table I for a summary of the differences between these Hamiltonians). As we wish to somewhat fairly compare different treatments of spin effects in the EOB formalism, we have modified some details of the original proposals of Refs. $[17,28]$ such that all the Hamiltonians agree in the zero-spin limit. We compare the aligned-spin circular-orbit binding energy functions from the different Hamiltonians with NR simulations and with the aligned-spin Hamiltonian from Refs. [19,23,47]. This enables one to assess compromises between accuracy and simplicity of the SEOB Hamiltonians.

The paper is organized as follows. In Sec. II, we provide an overview of SEOB Hamiltonians and their construction. Sections III and IV present the Ansätze of the SEOB Hamiltonians, with explicit results matched to 4PN in Appendix B. We then compare the aligned-spin circularorbit binding energy of the Hamiltonians against NR in Sec. V. Our conclusions are given in Sec. VI. Appendix A corrects an omission at NLO $\mathrm{S}^{2}$ in the Hamiltonian of Ref. [15]. 


\section{A. Notation}

We use geometric units such that the speed of light $c$ and the Newton constant $G$ are equal to 1 . We utilize various combinations of the masses $m_{1}, m_{2}$ of the binary's components,

$$
\begin{aligned}
& M=m_{1}+m_{2}, \quad \mu=\frac{m_{1} m_{2}}{M}, \quad \nu=\frac{\mu}{M}, \\
& q=\frac{m_{1}}{m_{2}}, \quad X_{1}=\frac{m_{1}}{M}, \quad X_{2}=\frac{m_{2}}{M} .
\end{aligned}
$$

For the spins $S_{1}, S_{2}$, we define the dimensionless versions

$$
\chi_{1}=\frac{\boldsymbol{a}_{1}}{m_{1}}=\frac{\boldsymbol{S}_{1}}{m_{1}^{2}}, \quad \chi_{2}=\frac{\boldsymbol{a}_{2}}{m_{2}}=\frac{\boldsymbol{S}_{2}}{m_{2}^{2}},
$$

along with the intermediate $\boldsymbol{a}_{1}, \boldsymbol{a}_{2}$. The relative position and momentum are denoted by $\boldsymbol{r}$ and $\boldsymbol{p}$, respectively. Using an implicit Euclidean background, it holds

$$
\boldsymbol{p}^{2}=p_{r}^{2}+\frac{L^{2}}{r^{2}}, \quad p_{r}=\boldsymbol{n} \cdot \boldsymbol{p}, \quad \boldsymbol{L}=\boldsymbol{r} \times \boldsymbol{p},
$$

where $\boldsymbol{n}=\boldsymbol{r} / r$ with $r=|\boldsymbol{r}|$, and $\boldsymbol{L}$ is the orbital angular momentum with magnitude $L$. For convenience, we also introduce rescaled dimensionless variables,

$$
\hat{\boldsymbol{r}}=\frac{\boldsymbol{r}}{M}, \quad \hat{\boldsymbol{p}}=\frac{\boldsymbol{p}}{\mu}, \quad \hat{H}=\frac{H}{\mu}, \quad \hat{\boldsymbol{L}}=\frac{\boldsymbol{L}}{M \mu}, \quad \hat{\boldsymbol{a}}=\frac{\boldsymbol{a}}{M}
$$

and similarly for the magnitudes $\hat{r}=|\hat{r}|$, etc. Here, $H$ is any of several Hamiltonians encountered below, and $\boldsymbol{a}=$ $S_{\text {Kerr }} / M$ is the rescaled spin of an effective Kerr black hole.

\section{SPINNING EFFECTIVE-ONE-BODY HAMILTONIANS}

In this section, we give an overview of spinning EOB Hamiltonians and their construction $[9,12-17,19,23$, $25,27,28,47]$, on which current EOB waveform models are built $[10,20,21,23,24,33-37,48]$. The EOB Hamiltonians are constructed such that (i) they describe geodesic motion in Kerr spacetime in the limit of vanishing mass ratio and that (ii) they agree (up to a canonical transformation) with a PN approximate Hamiltonian describing the conservative binary motion up to a certain order (here the 4PN order [49]). A certain class of EOB Hamiltonians [25,27,28] also incorporates the (nongeodesic) motion of spinning test particles in Kerr spacetime in the small-mass-ratio limit, as described by the Matthisson-Papapetrou-Dixon equations [50-54].

We consider a spinning binary in the center-of-mass frame. The orbital dynamics is described by the relative separation $\boldsymbol{r}$ and linear momentum $\boldsymbol{p}$ vectors, and the internal dynamics is assumed to be captured by the spins $S_{1}$ and $S_{2}$ of each body. The Poisson brackets between these dynamical variables are the standard ones,

$$
\begin{gathered}
\left\{r^{i}, p_{j}\right\}=\delta_{i j}, \\
\left\{S_{1}^{i}, S_{1}^{j}\right\}=\epsilon_{i j k} S_{1}^{k}, \\
\left\{S_{2}^{i}, S_{2}^{j}\right\}=\epsilon_{i j k} S_{2}^{k},
\end{gathered}
$$

with all others vanishing. The dynamics on phase space is generated by a Hamiltonian function $H\left(\boldsymbol{r}, \boldsymbol{p}, \boldsymbol{S}_{1}, \boldsymbol{S}_{2}\right)$. The equation of motion of a generic phase-space function $A$ reads

$$
\frac{d A}{d t}=\{A, H\}+\frac{\partial A}{\partial t} .
$$

Here the Hamiltonian is either the $\mathrm{PN} H^{\mathrm{PN}}$ or the EOB $H^{\mathrm{EOB}}$ one. The EOB Hamiltonian $H^{\mathrm{EOB}}$ itself is given in terms of another Hamiltonian, the effective Hamiltonian $H^{\text {eff }}$, via the energy map,

$$
H^{\mathrm{EOB}}=M \sqrt{1+2 \nu\left(\frac{H^{\mathrm{eff}}}{\mu}-1\right)} .
$$

The utility of this energy map was demonstrated, e.g., in Refs. $[9,43,55]$. For instance, if for $H^{\text {eff }}$ one just takes the Hamiltonian of geodesics in Schwarzschild spacetime, then $H^{\mathrm{EOB}}$ correctly describes both the $1 \mathrm{PN}$ and first postMinkowskian dynamics $[9,43]$.

\section{A. The effective Hamiltonian}

The central idea of the EOB Hamiltonian is to combine the dynamics in the test-body limit (with no restriction on the speed or field strength) with the PN dynamics (not restricted in the mass ratio). In this way, one might overcome some of the limitations of the individual approximations. This can be achieved by making an Ansatz for $H^{\text {eff }}$ as a deformation of the test-body-limit Hamiltonian (deforming it such that PN results are recovered), which is the purpose of this section. Note that in the test-body limit $H^{\mathrm{EOB}} \approx H^{\text {eff }}+$ const.

Let us review the Hamiltonian of a spinning test body in Kerr spacetime $[25,26]$. One can easily specialize this to the nonspinning (geodesic) case, which is the basis of some SEOB models. These test-body Hamiltonians are the basis for all SEOB models. The (inverse) Kerr metric $g_{\text {Kerr }}^{\mu \nu}$ in Boyer-Lindquist coordinates $\left(x^{\mu}\right)=(t, r, \theta, \phi)$ is given by the line element

$$
\begin{aligned}
-d \tau^{2}= & g_{\text {Kerr }}^{\mu \nu} \partial_{\mu} \partial_{\nu} \\
= & -\frac{\Lambda}{\Delta \Sigma} \partial_{t}^{2}+\frac{\Delta}{\Sigma} \partial_{r}^{2}+\frac{1}{\Sigma} \partial_{\theta}^{2} \\
& +\frac{\Sigma-2 M r}{\Sigma \Delta \sin ^{2} \theta} \partial_{\phi}^{2}-\frac{4 M r a}{\Sigma \Delta} \partial_{t} \partial_{\phi},
\end{aligned}
$$


where $M$ is the mass of the black hole, $\sigma=M a$ is its spin, and

$$
\begin{aligned}
& \Sigma \equiv r^{2}+a^{2} \cos ^{2} \theta, \quad \Delta \equiv r^{2}-2 M r+a^{2}, \\
& \Lambda \equiv\left(r^{2}+a^{2}\right)^{2}-a^{2} \Delta \sin ^{2} \theta .
\end{aligned}
$$

The Hamiltonian of a spinning test-body $H^{\text {Kerr }}$ can be obtained as a solution of the mass-shell constraint (see, e.g., Ref. [26]),

$$
\begin{aligned}
-\mu^{2}= & g_{\text {Kerr }}^{\mu \nu}\left(p_{\mu}-\frac{1}{2} \omega_{\mu a b} S_{*}^{a b}\right)\left(p_{\nu}-\frac{1}{2} \omega_{\mu a b} S_{*}^{a b}\right) \\
& +\mathcal{O}\left(S_{*}^{2}\right),
\end{aligned}
$$

where $p_{\mu}=\left(-H^{\text {Kerr }}, p_{r}, p_{\theta}, p_{\phi}\right), \mu$ is the mass of the test body, $S_{*}^{a b}=-S_{*}^{a b}$ is its spin tensor in a local Lorentz frame $\left(e_{a}^{\mu} e^{a \nu}=g_{\mathrm{Kerr}}^{\mu \nu}\right), \omega_{\mu a b}=e_{b \nu} \nabla_{\mu} e_{a}^{\nu}$ are the Ricci rotation coefficients, and $\nabla_{\mu}$ is the covariant derivative. The canonical spin vector of the test-body $\boldsymbol{S}_{*}$ is given by $S_{*}^{i}=\frac{1}{2} \epsilon^{i j k} S_{*}^{j k}$, and the components $S_{0 i}$ are fixed by the supplementary condition $S^{a b}\left(e_{b}{ }^{\mu} p_{\mu}+\mu^{2} \delta_{b}^{0}\right)=0+\mathcal{O}\left(S_{*}^{2}\right)$, all in the local frame.

Let us split $H^{\text {Kerr }}$ into a part dependent on the test-spin $S_{*}$ and the remaining $S_{*}$-independent terms into parts even and odd in the Kerr spin $a$,

$$
H^{\mathrm{Kerr}}=H_{\mathrm{even}}^{\mathrm{Kerr}}+H_{\mathrm{odd}}^{\mathrm{Kerr}}+H_{S_{*}}^{\mathrm{Kerr}} .
$$

Following the procedure outlined above, and choosing the local frame from Ref. [27], this leads to

$$
\begin{aligned}
H_{\mathrm{even}}^{\mathrm{Kerr}}= & \alpha^{\mathrm{Kerr}} \sqrt{\mu^{2}+\gamma_{\mathrm{Kerr}}^{\phi \phi} p_{\phi}^{2}+\gamma_{\mathrm{Kerr}}^{r r} p_{r}^{2}+\gamma_{\mathrm{Kerr}}^{\theta \theta} p_{\theta}^{2}}, \\
H_{\mathrm{odd}}^{\text {Kerr }}= & \beta^{\text {Kerr }} p_{\phi}, \\
H_{S_{*}}^{\mathrm{Kerr}}= & {\left[\boldsymbol{F}_{t}+\left(\beta^{\mathrm{Kerr}}+\frac{\alpha^{\mathrm{Kerr}} \gamma_{\mathrm{Kerr}}^{\phi \phi} p_{\phi}}{\sqrt{q^{\mathrm{Kerr}}}}\right) \boldsymbol{F}_{\phi}\right] \cdot \boldsymbol{S}_{*} } \\
& +\frac{\alpha^{\mathrm{Kerr}}}{\sqrt{q^{\mathrm{Kerr}}}}\left(\gamma_{\mathrm{Kerr}}^{r r} p_{r} \boldsymbol{F}_{r}+\gamma_{\mathrm{Kerr}}^{\theta \theta} p_{\theta} \boldsymbol{F}_{\theta}\right) \cdot \boldsymbol{S}_{*} \\
& +\mathcal{O}\left(S_{*}^{2}\right),
\end{aligned}
$$

with

$$
\begin{aligned}
& \alpha^{\mathrm{Kerr}}=\frac{1}{\sqrt{-g_{\text {Kerr }}^{t t}}}=\sqrt{\frac{\Delta \Sigma}{\Lambda}}, \\
& \beta^{\mathrm{Kerr}}=\frac{g_{\mathrm{Kerr}}^{t \phi}}{g_{\text {Kerr }}^{t t}}=\frac{2 a M r}{\Lambda},
\end{aligned}
$$

$$
\begin{aligned}
& \gamma_{\text {Kerr }}^{\phi \phi}=g_{\text {Kerr }}^{\phi \phi}-\frac{g_{\text {Kerr }}^{t \phi} g_{\text {Kerr }}^{t \phi}}{g_{\text {Kerr }}^{t t}}=\frac{\Sigma}{\Lambda \sin ^{2} \theta}, \\
& \gamma_{\text {Kerr }}^{r r}=g_{\text {Kerr }}^{r r}=\frac{\Delta}{\Sigma}, \\
& \gamma_{\text {Kerr }}^{\theta \theta}=g_{\text {Kerr }}^{\theta \theta}=\frac{1}{\Sigma}, \\
& \sqrt{q^{\text {Kerr }}}=\frac{H_{\mathrm{even}}^{\text {Kerr }}}{\alpha^{\text {Kerr }}},
\end{aligned}
$$

and with explicit expressions for the fictitious gravitomagnetic (frame-dragging) force interacting with the test-spin $\boldsymbol{S}_{*}$ given in Ref. [32] in terms of the vectors $\boldsymbol{F}_{\mu}$ (reproduced here in Sec. IV). A simplified version of this Hamiltonian for aligned spins and motion in the equatorial plane can be found in Ref. [56]. Simplifications for the generic-spin case are possible by making a different choice for the local frame which may simplify the Ricci rotation coefficients; see, e.g., Appendix C of Ref. [26].

The Hamiltonian above is written in terms of components instead of vectors, which is a disadvantage for some purposes. Following Ref. [17], we transform to a three-vector notation (with an implicit flat Euclidean background) by treating $(r, \theta, \phi)$ as spherical coordinates, with $\boldsymbol{r}=(x, y, z)=r(\sin \theta \cos \phi, \sin \theta \sin \phi, \cos \theta)$ and $\boldsymbol{a}=(0,0, a)$. This is accompanied by a transformation of the momenta $p_{r}, p_{\theta}, p_{\phi}$ to the new momenta $\boldsymbol{p}$,

$$
\begin{aligned}
& p_{r}=\boldsymbol{n} \cdot \boldsymbol{p}, \quad p_{\phi}=L_{z}=(\boldsymbol{r} \times \boldsymbol{p})_{z}, \\
& \frac{p_{\theta}^{2}}{r^{2}}=\boldsymbol{p}^{2}-p_{r}^{2}-\frac{p_{\phi}^{2}}{r^{2} \sin ^{2} \theta},
\end{aligned}
$$

which makes it an overall canonical transformation. Noting that $a^{2} p_{\phi}^{2} / r^{2}=(\boldsymbol{n} \times \boldsymbol{p} \cdot \boldsymbol{a})^{2}, a \cos \theta=\boldsymbol{n} \cdot \boldsymbol{a}$, and $a^{2} \sin ^{2} \theta=$ $a^{2}-(\boldsymbol{n} \cdot \boldsymbol{a})^{2}$, this results in the even-in- $a$ Hamiltonian,

$$
\begin{aligned}
H_{\mathrm{even}}^{\mathrm{Kerr}}= & {\left[A ^ { \mathrm { Kerr } } \left(\mu^{2}+B_{p}^{\mathrm{Kerr}} \boldsymbol{p}^{2}+B_{n p}^{\mathrm{Kerr}}(\boldsymbol{n} \cdot \boldsymbol{p})^{2}\right.\right.} \\
& \left.\left.+B_{n p a}^{\mathrm{Kerr}}(\boldsymbol{n} \times \boldsymbol{p} \cdot \boldsymbol{a})^{2}\right)\right]^{1 / 2},
\end{aligned}
$$

with

$$
A^{\mathrm{Kerr}}=\left(\alpha^{\mathrm{Kerr}}\right)^{2}=\frac{\Delta \Sigma}{\Lambda},
$$

$$
\begin{aligned}
B_{p}^{\mathrm{Kerr}} & =r^{2} \gamma_{\text {Kerr }}^{\theta \theta}=\frac{r^{2}}{\Sigma}, \\
B_{n p}^{\mathrm{Kerr}} & =\gamma_{\text {Kerr }}^{r r}-r^{2} \gamma_{\text {Kerr }}^{\theta \theta}=\frac{r^{2}}{\Sigma}\left[\frac{\Delta}{r^{2}}-1\right], \\
B_{n p a}^{\text {Kerr }} & =\frac{r^{2}}{a^{2}}\left[\gamma_{\text {Kerr }}^{\phi \phi}-\frac{\gamma_{\text {Kerr }}^{\theta \theta}}{\sin ^{2} \theta}\right]=-\frac{r^{2}}{\Sigma \Lambda}(\Sigma+2 M r)
\end{aligned}
$$


and

$$
\begin{aligned}
& \Sigma=r^{2}+(\boldsymbol{n} \cdot \boldsymbol{a})^{2}, \quad \Delta=r^{2}-2 M r+a^{2}, \\
& \Lambda=\left(r^{2}+a^{2}\right)^{2}-\Delta a^{2}+\Delta(\boldsymbol{n} \cdot \boldsymbol{a})^{2} .
\end{aligned}
$$

Similarly, the odd-in- $a$ part reads

$$
H_{\mathrm{odd}}^{\mathrm{Kerr}}=\beta^{\mathrm{Kerr}} p_{\phi}=\frac{2 M r}{\Lambda} \boldsymbol{L} \cdot \boldsymbol{a} .
$$

We now have all ingredients in order to discuss how an Ansatz for the effective Hamiltonian can be built. In general, one takes the effective Hamiltonian to be a deformation of the Kerr Hamiltonian (the deformation parameter being the symmetric mass ratio $\nu$ ), either for a test spin or a test mass. While all EOB models agree on the identification of the masses between the test body and comparable mass case $\left(M=m_{1}+m_{2}, \mu=m_{1} m_{2} / M\right)$, different choices are made for mapping the spins $\boldsymbol{a}$ and $\boldsymbol{S}_{*}$ to $\boldsymbol{S}_{1}$ and $\boldsymbol{S}_{2}$. Let us consider a simple explicit example. We could write the even-in- $a$ part of the effective Hamiltonian as

$$
\begin{aligned}
H_{\mathrm{even}}^{\mathrm{eff}}= & {\left[A \left(\mu^{2}+B_{p} \boldsymbol{p}^{2}+B_{n p}(\boldsymbol{n} \cdot \boldsymbol{p})^{2}\right.\right.} \\
& \left.\left.+B_{n p a}(\boldsymbol{n} \times \boldsymbol{p} \cdot \boldsymbol{a})^{2}+\mu^{2} Q\right)\right]^{1 / 2},
\end{aligned}
$$

where the momentum-independent potentials $A, B_{p}, B_{n p}$, $B_{n p a}$ are the Kerr potentials given above modified by PN corrections (to be determined). The quantity $Q$ is a momentum-dependent potential introduced in Ref. [55], which may accommodate PN terms that do not fit into the momentum-independent potentials. (In cases where $Q$ vanishes, the deformed Hamiltonian can be interpreted as describing geodesic motion in a $\nu$-deformed Kerr metric.) The mentioned potentials should all be of even order in spin, while terms of odd order in spin should be included via a deformation of $H_{\mathrm{odd}}^{\mathrm{Kerr}}$. More explicit Ansätze for the PN-corrected SEOB Hamiltonians and their potentials are discussed below.

\section{B. Matching to post-Newtonian results}

To fix the potentials in the Ansatz for an effective Hamiltonian, one demands that the EOB Hamiltonian $H^{\mathrm{EOB}}$ agrees with the Hamiltonian in the PN approximation $H^{\mathrm{PN}}$ up to a canonical transformation. This will eventually not uniquely fix the potentials, but leave some (gauge) freedom.

Here we use the spinning PN Hamiltonian derived in the framework and gauges introduced in Ref. [57], since it is available to 4PN order in the spinning sector [49]. Broken up into LO, NLO, NNLO PN parts and into powers of spin, it reads

$$
\begin{aligned}
& H_{S}^{\mathrm{LO}}+H_{S}^{\mathrm{NLO}}+H_{S}^{\mathrm{NNLO}} \\
& +H_{S^{2}}^{\mathrm{LO}} \quad+H_{S^{2}}^{\mathrm{NLO}} \quad+H_{S^{2}}^{\mathrm{NNLO}} \\
& +H_{S^{3}}^{\mathrm{LO}} \\
& H_{\mathrm{spin}}^{\mathrm{PN}}=\quad+H_{S^{4}}^{\mathrm{LO}} \\
& \mathcal{O}\left(\frac{1}{c^{3}}\right)+\mathcal{O}\left(\frac{1}{c^{4}}\right)+\mathcal{O}\left(\frac{1}{c^{5}}\right)+\mathcal{O}\left(\frac{1}{c^{6}}\right)+\mathcal{O}\left(\frac{1}{c^{7}}\right)+\mathcal{O}\left(\frac{1}{c^{8}}\right),
\end{aligned}
$$

where columns correspond to PN orders counted by the inverse of the speed of light $c$ (one PN order is $\mathcal{O}\left(c^{-2}\right)$ ). Except for the self-spin-squared interactions in $H_{S^{2}}^{\mathrm{NNLO}}$ calculated in Ref. [58], these results have been derived in different frameworks and checked against each other: $H_{S}^{\mathrm{LO}}$ in Refs. [59-66], $H_{S}^{\mathrm{NLO}}$ in Refs. [67-73], $H_{S}^{\mathrm{NNLO}}$ in Refs. [74-78], $H_{S^{2}}^{\mathrm{LO}}$ in Refs. [61-63,65,79], $H_{S^{2}}^{\mathrm{NLO}}$ in Refs. [80-88], $H_{S^{2}}^{\mathrm{NNLO}}$ in Refs. [58,75,89-91], $H_{S^{3}}^{\mathrm{LO}}$ in Refs. [92-96], and $H_{S^{4}}^{\mathrm{LO}}$ in Refs. [92-94,96]. These Hamiltonians are valid for both black holes and neutron stars. They depend on coefficients $\left(\tilde{C}_{\left(\mathrm{ES}^{2}\right)}, \tilde{C}_{\left(\mathrm{BS}^{3}\right)}, \tilde{C}_{\left(\mathrm{ES}^{4}\right)}\right)$ which are the proportionality constants between the spininduced multipoles (quadrupole, octupole, hexadecapole) and symmetric-tracefree tensors built out of (two, three, four) spin vectors (respectively). The proportionality constants depend on the type of compact object (and on the equation of state in case of a neutron star); here they are normalized to zero for black holes (in the original paper [49], they are normalized to one and denoted without a tilde; see also Appendix B). This normalization makes sense here since we base the EOB Hamiltonian on a deformation of the Kerr one. Of course, the PN Hamiltonian $H^{\mathrm{PN}}=$ $H_{\mathrm{ns}}^{\mathrm{PN}}+H_{\text {spin }}^{\mathrm{PN}}$ must be supplemented by its nonspinning (ns) part $H_{\mathrm{ns}}^{\mathrm{PN}}$, which we only need to $2 \mathrm{PN}$ order here in order to construct the canonical transformation of the spin sector; it can be derived, e.g., from the Lagrangian in Ref. [97]. The nonspinning part was derived to 4PN order using independent methods [98-101] and partial results at 5PN have already been obtained [102-104].

The condition that the EOB Hamiltonian $H^{\mathrm{EOB}}$ must coincide with results for the $\mathrm{PN}$-approximate binary Hamiltonian $H^{\mathrm{PN}}$ up to a canonical transformation reads

$$
\begin{aligned}
H^{\mathrm{EOB}}= & H^{\mathrm{PN}}+\left\{\mathcal{G}, H^{\mathrm{PN}}\right\}+\frac{1}{2 !}\left\{\mathcal{G},\left\{\mathcal{G}, H^{\mathrm{PN}}\right\}\right\} \\
& +\frac{1}{3 !}\left\{\mathcal{G},\left\{\mathcal{G},\left\{\mathcal{G}, H^{\mathrm{PN}}\right\}\right\}\right\}+\ldots,
\end{aligned}
$$

where $\mathcal{G}$ is the generating function of the canonical transformation. If $\mathcal{G}$ is small in the PN approximation, then the series in Eq. (21) terminates after a finite number of terms at a given PN order. In practice, one makes a PN approximate and manifestly rotation invariant Ansatz for $\mathcal{G}$ in terms of 
the canonical variables; we provide an explicit expression for $\mathcal{G}$ as Mathematica code in the Supplemental Material [105]. Equation (21) then leads to constraints on the coefficients in the Ansatz for $\mathcal{G}$ and $H^{\text {eff }}$. The remaining freedom in the coefficients is a gauge freedom within the EOB formalism.

Let us note some general considerations about how part of this gauge freedom can be fixed in SEOB models. Since binaries are expected to be on almost circular orbits during their last orbits, it makes sense to fix the gauge freedom of the EOB Hamiltonian such that it simplifies for circular orbits, for which $p_{r} \equiv \boldsymbol{n} \cdot \boldsymbol{p}=0$ [55]. Taking the Ansatz in Eq. (19) as an example, this means that-using the canonical transformation discussed above-one should transform as many PN terms as possible into a form such that they can be included in the potential $B_{n p}^{\text {Kerr }}$, which drops out of the Hamiltonian for circular orbits. In the nonspinning case, it is additionally possible to require that the potential $Q$ depends on the momentum only via $p_{r}$, and this uniquely fixes all EOB gauge freedom $[9,55]$. For the example in Eq. (19), following the structure of the nonspinning Hamiltonian, it is natural to require that (i) the momentum dependence of $H_{\mathrm{odd}}^{\mathrm{Kerr}}$ is expressed in terms of $p_{r}$ whenever possible [13], (ii) $B_{n p a}=B_{n p a}^{\text {Kerr }}$ [17], and (iii) terms in $Q$ have a power in $p_{r}$ that is as high as possible. The last requirement ensures that $Q$ vanishes for circular orbits, as in the nonspinning case.

These considerations still leave some remaining gauge freedom in the spinning case, which we fix such to simplify the EOB Hamiltonian also for aligned spins. For example, it is possible to choose PN corrections in the potential $B_{p}$ such that it only depends on terms of the form $\boldsymbol{n} \cdot \boldsymbol{S}$ but not $S \cdot S$. Any remaining gauge freedom beyond that may be chosen arbitrarily.

\section{SPINNING EFFECTIVE-ONE-BODY HAMILTONIANS WITH TEST MASS}

In this section, we present different Ansätze for effective Hamiltonians based on the Kerr-geodesic one. That is, we do not include the Kerr test-spin Hamiltonian $H_{S_{*}}^{\text {Kerr }}$ here and instead make an Ansatz of the form

$$
H^{\mathrm{eff}}=H_{\mathrm{even}}^{\mathrm{eff}}+H_{\mathrm{odd}}^{\mathrm{eff}} \text {. }
$$

The explicit lengthy results from the matching at 4PN order against PN results (and fixing of the remaining gauge freedom) are given in Appendix B. We start with an extension of the SEOB Hamiltonian from Ref. [17], which we call $\mathrm{SEOB}_{\mathrm{TM}}^{r_{c}}$, to $4 \mathrm{PN}$ order, here including spin effects at LO $\mathrm{S}^{3}$, and NNLO $\mathrm{S}^{2}$. We also extend that Hamiltonian from black holes to generic compact objects, e.g., neutron stars. We proceed with a simplified version of the $\mathrm{SEOB}_{\mathrm{TM}}^{r_{c}}$ Hamiltonian to $4 \mathrm{PN}$ order that does not make use of the centrifugal radius introduced in Ref. [19].
For completeness, we also summarize the $\mathrm{SEOB}_{\mathrm{TM}}^{r_{c} \text { align }}$ Hamiltonian from Refs. $[19,22,23,47]$ which is valid for aligned spins only. We do not include additional PN terms in the $\mathrm{SEOB}_{\mathrm{TM}}^{r_{c} \text {,align }}$ Hamiltonian since it is already $4 \mathrm{PN}$ complete for generic bodies. For convenience, we summarize the Hamiltonians in Table I.

\section{A. Effective-one-body Hamiltonian with test-mass limit and centrifugal radius: $\mathrm{SEOB}_{\mathrm{TM}}^{r_{c}}$}

Reference [17] was the first to construct an SEOB Hamiltonian with NLO spin-squared terms for generic spin orientations (but omitting a subtle contribution, included here, see Appendix A). Here we extend the Hamiltonian to include NNLO spin-squared and LO spin-cubed terms, and add multipole constants to make it applicable to generic bodies like neutron stars.

For the even-in-spin part of the $\mathrm{SEOB}_{\mathrm{TM}}^{r_{c}}$ Hamiltonian, we use the Ansatz in Eq. (19),

$$
\begin{aligned}
H_{\mathrm{even}}^{\mathrm{eff}}= & {\left[A \left(\mu^{2}+B_{p} \boldsymbol{p}^{2}+B_{n p}(\boldsymbol{n} \cdot \boldsymbol{p})^{2}\right.\right.} \\
& \left.\left.+B_{n p a}(\boldsymbol{n} \times \boldsymbol{p} \cdot \boldsymbol{a})^{2}+\mu^{2} Q\right)\right]^{1 / 2},
\end{aligned}
$$

where the Kerr spin is mapped according to

$$
a=a_{1}+a_{2}
$$

This ensures that the Hamiltonian reproduces leading-order PN results at all even orders in spin [106]. The effective Hamiltonian further uses the centrifugal radius $r_{c}$, which was introduced in Ref. [19], and is defined such that the Kerr Hamiltonian for aligned spins and equatorial orbits can be written as $H_{\mathrm{even}}^{\mathrm{Kerr}}=\sqrt{A^{\mathrm{Kerr}}\left(\mu^{2}+p_{\phi}^{2} / r_{c}^{2}+p_{r}^{2} / B(r)\right)}$, which implies the definition

$$
r_{c}=\sqrt{r^{2}+a^{2}+\frac{2 M a^{2}}{r}}
$$

The centrifugal radius was generalized to generic spin orientations in Ref. [17]. In terms of $r_{c}$, the Kerr potential $A^{\text {Kerr }}$ from Eq. (16a) can be written equivalently as

$$
A^{\mathrm{Kerr}}=\left(1-\frac{2 M}{r_{c}}\right) \frac{\left(1+\frac{2 M}{r_{c}}\right)}{\left(1+\frac{2 M}{r}\right)} \frac{1+\frac{(\boldsymbol{n} \cdot \boldsymbol{a})^{2}}{r^{2}}}{1+\Delta \frac{(\boldsymbol{n} \cdot \boldsymbol{a})^{2}}{r^{2} r_{c}^{2}}} .
$$

In the nonspinning limit, only the first term above remains, which reduces to the Schwarzschild $A$ potential. This is the reason why Ref. [17] adds the zero-spin PN corrections to $1-2 M / r_{c}$. However, in this paper, we intend to investigate spin effects across different Hamiltonian descriptions, so we need to make sure that the nonspinning Hamiltonians are identical. That is, we need to choose a method for adding zero-spin corrections that can be applied to all four 
EOB Hamiltonians considered here. We simply multiply the Kerr potential $A^{\text {Kerr }}$ by zero-spin PN corrections denoted as $A^{0}$ below (without performing a Padé or log resummation ${ }^{3}$ of $A^{0}$ ). For the spin-squared corrections, we follow Ref. [17] and add spin-squared corrections of the form $\boldsymbol{n} \cdot \boldsymbol{S}$ to the term $1+(\boldsymbol{n} \cdot \boldsymbol{a})^{2} / r^{2}$, and add corrections of the form $\boldsymbol{S} \cdot \boldsymbol{S}$ to $1+2 M / r_{c}$, since it has an expansion of the form $1+2 M / r-M a^{2} / r^{3}+\cdots$. One employs similar considerations for adding $\mathrm{PN}$ corrections to the $B$ potentials in Eq. (23), leading to the following Ansatz:

$$
\begin{aligned}
A & =\left(1-\frac{2 M}{r_{c}}\right) \frac{\left(1+\frac{2 M}{r_{c}}+A^{S S}+A^{S^{4}}\right)}{\left(1+\frac{2 M}{r}\right)} \frac{\left(1+\frac{(\boldsymbol{n} \cdot \boldsymbol{a})^{2}}{r^{2}}+A^{n S}\right)}{\left(1+\Delta \frac{(\boldsymbol{n} \cdot \boldsymbol{a})^{2}}{r^{2} r_{c}^{2}}\right)} A^{0}\left(r_{c}\right), \\
B_{p} & =\left[1+\frac{(\boldsymbol{n} \cdot \boldsymbol{a})^{2}}{r^{2}}+B_{p}^{n S}\right]^{-1}, \\
B_{n p} & =\frac{1}{1+\frac{(\boldsymbol{n} \cdot \boldsymbol{a})^{2}}{r^{2}}}\left[\left(1-\frac{2 M}{r}+\frac{a^{2}}{r^{2}}\right)\left(A^{0}\left(r_{c}\right) D^{0}\left(r_{c}\right)+B_{n p}^{S S}+B_{n p}^{n S}\right)-1\right], \\
B_{n p a} & =B_{n p a}^{\mathrm{Kerr}}, \\
Q & =Q^{0}\left(r_{c}\right)+Q^{S^{2}} .
\end{aligned}
$$

Note that we use the gauge choice from Ref. [17], i.e., there are no corrections of the form $\boldsymbol{S} \cdot \boldsymbol{S}$ in the potential $B_{p}$, which simplifies the Hamiltonian for aligned spins and circular orbits.

The 4PN corrections to the nonspinning effective Hamiltonian were obtained in Ref. [107]. Since we factor the PN corrections in $A^{0}\left(r_{c}\right)$, we choose it such that the PN expansion of the $A$ potential agrees, in the nonspinning limit, with the results of Ref. [107]. Writing the PN corrections using scaled variables (4) to simplify notation, we obtain

$$
\begin{aligned}
A^{0}\left(r_{c}\right)= & +\nu\left[\frac{2}{\hat{r}_{c}^{3}}+\left(\frac{106}{3}-\frac{41}{32} \pi^{2}\right) \frac{1}{\hat{r}_{c}^{4}}+\left(\frac{1}{20}+\frac{41}{32} \pi^{2} \nu-\frac{221}{6} \nu+\frac{963}{512} \pi^{2}+\frac{128}{5} \gamma_{E}+\frac{256}{5} \ln 2+\frac{64}{5} \ln \frac{1}{\hat{r}_{c}}\right) \frac{1}{\hat{r}_{c}^{5}}\right], \\
D^{0}\left(r_{c}\right)= & +6 \nu \frac{1}{\hat{r}_{c}^{2}}+\left(52 \nu-6 \nu^{2}\right) \frac{1}{\hat{r}_{c}^{3}} \\
& +\left[\left(\frac{123}{16} \pi^{2}-260\right) \nu^{2}+\nu\left(-\frac{23761}{1536} \pi^{2}-\frac{533}{45}+\frac{1184}{15} \gamma_{E}-\frac{6496}{15} \ln 2+\frac{2916}{5} \ln 3\right)+\frac{592}{15} \nu \ln \frac{1}{\hat{r}_{c}}\right] \frac{1}{\hat{r}_{c}^{4}}, \\
Q^{0}\left(r_{c}\right)= & {\left[2(4-3 \nu) \nu \frac{1}{\hat{r}_{c}^{2}}+\left(\left(-\frac{5308}{15}+\frac{496256}{45} \ln 2-\frac{33048}{5} \ln 3\right) \nu-83 \nu^{2}+10 \nu^{3}\right) \frac{1}{\hat{r}_{c}^{3}}\right] \hat{p}_{r}^{4} } \\
& +\left[\left(-\frac{827}{3}-\frac{2358912}{25} \ln 2+\frac{1399437}{50} \ln 3+\frac{390625}{18} \ln 5\right) \nu-\frac{27}{5} \nu^{2}+6 \nu^{3}\right] \frac{\hat{p}_{r}^{6}}{\hat{r}_{c}^{2}},
\end{aligned}
$$

where the corrections are expressed in terms of the centrifugal radius $r_{c}$, with $\hat{r}_{c}=r_{c} / M$, and $\hat{p}_{r}=p_{r} / \mu$.

\footnotetext{
${ }^{3}$ The justification for the Padé or log resummations is that they improve agreement with NR in some models and may hence be seen as an implicit calibration. In this paper, however, we consider EOB Hamiltonians with no calibration to NR, so we try to avoid such resummations, in particular in the nonspinning part.
}

Note that here and in the SEOB models discussed below, we are using Taylor expanded and not resummed versions of these potentials-we want to compare the different Ansätze of the Hamiltonians irrespective of possible resummations for the potentials (see also footnote 3).

Spin-squared contributions, up to NNLO, are added to the Hamiltonian using the following Ansatz: 


$$
\begin{aligned}
A^{S S}= & \frac{c_{n}}{\hat{r}_{c}^{3}} \chi_{i} \cdot \chi_{j}+\frac{c_{n}}{\hat{r}_{c}^{4}} \chi_{i} \cdot \chi_{j}+\frac{c_{n}}{\hat{r}_{c}^{5}} \chi_{i} \cdot \chi_{j}, \\
A^{n S}= & \frac{c_{n}}{\hat{r}_{c}^{3}}\left(\boldsymbol{n} \cdot \chi_{i}\right)\left(\boldsymbol{n} \cdot \chi_{j}\right)+\frac{c_{n}}{\hat{r}_{c}^{4}}\left(\boldsymbol{n} \cdot \chi_{i}\right)\left(\boldsymbol{n} \cdot \chi_{j}\right) \\
& +\frac{c_{n}}{\hat{r}_{c}^{5}}\left(\boldsymbol{n} \cdot \chi_{i}\right)\left(\boldsymbol{n} \cdot \chi_{j}\right), \\
B_{p}^{n S}= & \frac{c_{n}}{\hat{r}_{c}^{3}}\left(\boldsymbol{n} \cdot \chi_{i}\right)\left(\boldsymbol{n} \cdot \chi_{j}\right)+\frac{c_{n}}{\hat{r}_{c}^{4}}\left(\boldsymbol{n} \cdot \chi_{i}\right)\left(\boldsymbol{n} \cdot \chi_{j}\right), \\
B_{n p}^{n S}= & \frac{c_{n}}{\hat{r}_{c}^{3}}\left(\boldsymbol{n} \cdot \chi_{i}\right)\left(\boldsymbol{n} \cdot \chi_{j}\right)+\frac{c_{n}}{\hat{r}_{c}^{4}}\left(\boldsymbol{n} \cdot \chi_{i}\right)\left(\boldsymbol{n} \cdot \chi_{j}\right), \\
B_{n p}^{S S}= & \frac{c_{n}}{\hat{r}_{c}^{3}} \chi_{i} \cdot \chi_{j}+\frac{c_{n}}{\hat{r}_{c}^{4}} \chi_{i} \cdot \chi_{j}, \\
Q^{S^{2}}= & \frac{\hat{p}_{r}^{4}}{\hat{r}_{c}^{3}}\left[c_{n} \chi_{i} \cdot \chi_{j}+c_{n}\left(\boldsymbol{n} \cdot \chi_{i}\right)\left(\boldsymbol{n} \cdot \chi_{j}\right)\right] \\
& +\frac{\hat{p}_{r}^{3}}{\hat{r}_{c}^{3}} c_{n}\left(\boldsymbol{p} \cdot \chi_{i}\right)\left(\boldsymbol{n} \cdot \chi_{j}\right),
\end{aligned}
$$

where we followed Ref. [17] in expressing the corrections in terms of $r_{c}$. We employ notation such that, e.g., $c_{n} \chi_{i} \cdot \chi_{j} \equiv c_{n} \chi_{1}^{2}+c_{n} \chi_{1} \cdot \chi_{2}+c_{n} \chi_{2}^{2}$. Each $c_{n}$ stands for an independent undetermined coefficient in our Ansatz, i.e., we use the same symbol $c_{n}$ for all coefficients to simplify notation. The full expressions after matching to PN results are provided in Appendix B. Note that we added LO $S^{2}$ corrections to the $A$ potential above (which vanish for black holes) to account for the multipole constants of neutron stars.

The NLO S $S^{2}$ contributions were included in the effective Hamiltonian in Ref. [17]; however, the authors missed a contribution in matching the EOB Hamiltonian to PN results, namely, from the $\mathrm{LO} \mathrm{S}^{2}$ generating function applied to the LO SO Hamiltonian, i.e., from the Poisson bracket $\left\{\mathcal{G}_{S^{2}}^{\mathrm{LO}}, H_{S}^{\mathrm{LO}}\right\}$. In Appendix A, we write the matching results for NLO $S^{2}$, using the notation of Ref. [17], after taking into account the missing Poisson bracket.

The leading-order quartic-in-spin terms $A^{S^{4}}$ are zero for black holes, since the Kerr Hamiltonian, with the mapping $\boldsymbol{a}=\boldsymbol{a}_{1}+\boldsymbol{a}_{2}$, automatically reproduces them, but they are nonzero for other types of compact objects. We take the most generic expression for the $\mathrm{S}^{4}$ corrections,

$$
\begin{aligned}
A^{S^{4}}= & \frac{1}{\hat{r}_{c}^{5}}\left[c_{n}\left(\chi_{i} \cdot \chi_{j}\right)\left(\chi_{k} \cdot \chi_{l}\right)\right. \\
& +c_{n}\left(\chi_{i} \cdot \chi_{j}\right)\left(\boldsymbol{n} \cdot \chi_{k}\right)\left(\boldsymbol{n} \cdot \chi_{l}\right) \\
& \left.+c_{n}\left(\boldsymbol{n} \cdot \chi_{i}\right)\left(\boldsymbol{n} \cdot \chi_{j}\right)\left(\boldsymbol{n} \cdot \chi_{k}\right)\left(\boldsymbol{n} \cdot \chi_{l}\right)\right],
\end{aligned}
$$

where a summation over the spins of the two bodies is implied, and terms symmetric under the exchange of the two bodies' labels are only included once.

The spin-orbit and spin-cubed PN corrections are added to the odd-in-spin part of the Kerr Hamiltonian $H_{\text {odd }}^{\text {Kerr }}$. For the SO part, we use the Ansatz in Refs. [17,19], and we add to it $S^{3}$ corrections,

$$
\begin{aligned}
\hat{H}_{\mathrm{odd}}^{\mathrm{eff}}= & \frac{G_{S}}{\hat{r} \hat{r}_{c}^{2}\left(1+\Delta \frac{(\boldsymbol{n} \cdot \boldsymbol{a})^{2}}{r^{2} r_{c}^{2}}\right)}\left(X_{1}^{2} \hat{\boldsymbol{L}} \cdot \chi_{1}+X_{2}^{2} \hat{\boldsymbol{L}} \cdot \chi_{2}\right) \\
& +\frac{G_{S^{*}}}{\hat{r}_{c}^{3}} \nu\left(\hat{\boldsymbol{L}} \cdot \chi_{1}+\hat{\boldsymbol{L}} \cdot \chi_{2}\right) \\
& +\frac{G_{S^{3}}}{\hat{r}_{c}^{4}} \hat{\boldsymbol{L}} \cdot \chi_{1}+\frac{\tilde{G}_{S^{3}}}{\hat{r}_{c}^{4}} \hat{\boldsymbol{L}} \cdot \chi_{2},
\end{aligned}
$$

where

$$
\begin{aligned}
G_{S}= & 2\left[1+\frac{c_{n}}{\hat{r}_{c}}+c_{n} \hat{p}_{r}^{2}+\frac{c_{n}}{\hat{r}_{c}^{2}}+c_{n} \frac{\hat{p}_{r}^{2}}{\hat{r}_{c}}+c_{n} \hat{p}_{r}^{4}\right]^{-1}, \\
G_{S^{*}}= & \frac{3}{2}\left[1+\frac{c_{n}}{\hat{r}_{c}}+c_{n} \hat{p}_{r}^{2}+\frac{c_{n}}{\hat{r}_{c}^{2}}+c_{n} \frac{\hat{p}_{r}^{2}}{\hat{r}_{c}}+c_{n} \hat{p}_{r}^{4}\right]^{-1} \quad \text { (32a) } \\
G_{S^{3}}= & \frac{1}{\hat{r}_{c}}\left[c_{n} \chi_{1} \cdot \chi_{1}+c_{n} \chi_{2} \cdot \chi_{2}+c_{n} \chi_{1} \cdot \chi_{2}\right. \\
& \left.+c_{n}\left(\boldsymbol{n} \cdot \chi_{1}\right)^{2}+c_{n}\left(\boldsymbol{n} \cdot \chi_{2}\right)^{2}+c_{n}\left(\boldsymbol{n} \cdot \chi_{1}\right)\left(\boldsymbol{n} \cdot \chi_{2}\right)\right] \\
& +\hat{p}_{r}^{2}\left[c_{n}\left(\boldsymbol{n} \cdot \chi_{1}\right)^{2}+c_{n}\left(\boldsymbol{n} \cdot \chi_{2}\right)^{2}+c_{n}\left(\boldsymbol{n} \cdot \chi_{1}\right)\left(\boldsymbol{n} \cdot \chi_{2}\right)\right] \\
& +\frac{\hat{L}^{2}}{\hat{r}^{2}}\left[c_{n}\left(\boldsymbol{n} \cdot \chi_{1}\right)^{2}+c_{n}\left(\boldsymbol{n} \cdot \chi_{2}\right)^{2}+c_{n}\left(\boldsymbol{n} \cdot \chi_{1}\right)\left(\boldsymbol{n} \cdot \chi_{2}\right)\right], \\
\tilde{G}_{S^{3}}= & G_{S^{3}} \quad \text { with } \quad 1 \leftrightarrow 2 .
\end{aligned}
$$

Note that an inverse-Taylor resummation is used for $G_{S}$ and $G_{S^{*}}$, which improves the description of the binary dynamics for aligned spins [19]. In the spin-cubed corrections $G_{S^{3}}$ and $\tilde{G}_{S^{3}}$, a gauge freedom exists which we chose such that terms of the form $p_{r}^{2} \chi_{i} \cdot \chi_{j}$ or $L^{2} \chi_{i} \cdot \chi_{j}$ are not included. Explicit results after matching at $4 \mathrm{PN}$ can be found in Appendix B 1.

\section{B. A simplified effective-one-body Hamiltonian with test-mass limit: $\mathrm{SEOB}_{\mathrm{TM}}$}

Since it is important to have fast and simple EOB waveform models, in this section, we consider a simplified version of the $\mathrm{SEOB}_{\mathrm{TM}}^{r_{c}}$ Hamiltonian that uses $r$ instead of $r_{c}$ for the PN corrections. In order to assess the effect of this simplification, we also avoid resummations that are not motivated by the structure of the interactions, i.e., we factorize spin corrections to the Kerr potentials and do not use an inverse-Taylor resummation for the spin-orbit part.

The potentials of the effective Hamiltonian are simply taken to be 


$$
\begin{aligned}
A & =A^{\operatorname{Kerr}}\left(A^{0}+A^{S S}+A^{n S}+A^{S^{4}}\right) \\
B_{p} & =B_{p}^{\operatorname{Kerr}}\left(1+B_{p}^{n S}\right) \\
B_{n p} & =\frac{\left(1-\frac{2}{\hat{r}}+\frac{\hat{a}^{2}}{\hat{r}^{2}}\right)\left(A^{0} D^{0}+B_{n p}^{S S}+B_{n p}^{n S}\right)-1}{1+(\boldsymbol{n} \cdot \hat{\boldsymbol{a}})^{2} / \hat{r}^{2}} \\
B_{n p a} & =B_{n p a}^{\mathrm{Kerr}} \\
Q & =Q^{0}+Q^{S^{2}}
\end{aligned}
$$

where the zero-spin corrections $A^{0}(r), D^{0}(r)$, and $Q^{0}(r)$ are given by Eq. (28) but in terms of $r$ instead of $r_{c}$. The Ansätze for the $\mathrm{S}^{2}$ and $\mathrm{S}^{4}$ corrections are given by the corresponding expressions from the previous section, i.e., Eqs. (29) and (30), but using $r$ instead of $r_{c}$ (and with different coefficients $c_{n}$ ). For $H_{\text {odd }}^{\mathrm{Kerr}}$, we modify the odd-in$a$ part of the Kerr Hamiltonian by the $\mathrm{SO}$ and $\mathrm{S}^{3} \mathrm{PN}$ corrections, that is,

$$
\begin{aligned}
\hat{H}_{\mathrm{odd}}^{\mathrm{eff}}= & \frac{1}{\hat{r} \hat{r}_{c}^{2}\left(1+\Delta \frac{(\boldsymbol{n} \cdot \boldsymbol{a})^{2}}{r^{2} r_{c}^{2}}\right)}\left[G_{S}\left(X_{1}^{2} \hat{\boldsymbol{L}} \cdot \chi_{1}+X_{2}^{2} \hat{\boldsymbol{L}} \cdot \chi_{2}\right)\right. \\
& +G_{S^{*}} \nu\left(\hat{\boldsymbol{L}} \cdot \chi_{1}+\hat{\boldsymbol{L}} \cdot \chi_{2}\right)+\frac{\left.G_{S^{3}} \hat{\boldsymbol{L}} \cdot \chi_{1}+\frac{\tilde{G}_{S^{3}}}{\hat{r}} \hat{\boldsymbol{L}} \cdot \chi_{2}\right],}{}
\end{aligned}
$$

with the Ansätze for the coefficients given in Eqs. (32a) and (32b), but again written with $r$ instead of $r_{c}$ (and different $\left.c_{n}\right)$. Explicit results after matching at $4 \mathrm{PN}$ can be found in Appendix B 2.

\section{Aligned effective-one-body Hamiltonian with test-mass limit and centrifugal radius: $\mathrm{SEOB}_{\mathrm{TM}}^{r_{c}, \text { align }}$}

In this section, we consider the aligned-spin EOB Hamiltonian proposed by Damour and Nagar in Ref. [19] and extended in Refs. [22,23,47], which we denote $\mathrm{SEOB}_{\mathrm{TM}}^{r_{c} \text {,align }}$. That Hamiltonian is similar to the aligned-spin limit of the $\mathrm{SEOB}_{\mathrm{TM}}^{r_{c}}$ Hamiltonian from above, except that the even-in-spin PN corrections are added to the centrifugal radius.

The even-in-spin effective Hamiltonian is given by

$$
\hat{H}_{\mathrm{even}}^{\mathrm{eff}}=\sqrt{A\left(1+\frac{\hat{L}^{2}}{\hat{r}_{c}^{2}}+\frac{\hat{p}_{r}^{2}}{B}+Q^{0}\right)} .
$$

The EOB potentials $A$ and $B$ are given in Ref. [19], but we do not use Padé resummation and we modify how the zerospin PN corrections are added such that they agree with the other Hamiltonians in this paper, that is,

$$
\begin{aligned}
& A=\left(1-\frac{2}{\hat{r}_{c}}\right) \frac{1+\frac{2}{\hat{r}_{c}}}{1+\frac{2}{\hat{r}_{c}}} A^{0}\left(r_{c}\right), \\
& B=\frac{r^{2}}{r_{c}^{2}} \frac{1}{A D^{0}\left(r_{c}\right)},
\end{aligned}
$$

where $A^{0}, D^{0}$, and $Q^{0}$ are given by Eq. (28). Note that Refs. $[23,46,47]$ use $Q \equiv 2 \nu(4-3 \nu) p_{r}^{4} / r_{c}^{2}$ instead of $Q^{0}$, and use $p_{r_{*}} \equiv p_{r} \sqrt{A / B}$ instead of $p_{r}$.

The spin-squared and spin-quartic corrections are added to the centrifugal radius, which is here defined by

$\hat{r}_{c}^{2}=\hat{r}^{2}+\hat{a}_{Q}^{2}\left(1+\frac{2}{\hat{r}}\right)+\frac{\delta a_{\mathrm{NLO}}^{2}}{\hat{r}}+\frac{\delta a_{\mathrm{NNLO}}^{2}}{\hat{r}^{2}}+\frac{\delta a_{\mathrm{LO}}^{4}}{\hat{r}^{2}}$,

and where $\hat{a}_{Q}$ depends on the compact object's multipolar constants,

$$
\hat{a}_{Q}^{2} \equiv \hat{a}^{2}+\tilde{C}_{1\left(E S^{2}\right)} \hat{a}_{1}^{2}+\tilde{C}_{2\left(E S^{2}\right)} \hat{a}_{2}^{2}
$$

where $\hat{a}_{i}=a_{i} / M, \hat{a}=|\boldsymbol{a}| / M$, and recalling Eq. (24).

The spin-orbit part was obtained in Ref. [19] with NNNLO $\nu$-independent spinning-test-body contributions and $\nu$-dependent contributions calibrated to NR. We do not include those higher-order corrections here, but we follow Ref. [19] in using an inverse-Taylor resummation/calibration of the coefficients $G_{S}$ and $G_{S^{*}}$ in

$$
\begin{aligned}
\hat{H}_{\mathrm{odd}}^{\text {eff }}= & \frac{G_{S}}{\hat{r} \hat{r}_{c}^{2}}\left(X_{1}^{2} \hat{L} \chi_{1}+X_{2}^{2} \hat{L} \chi_{2}\right)+\frac{G_{S^{*}}}{\hat{r}_{c}^{3}} \nu\left(\hat{L} \chi_{1}+\hat{L} \chi_{2}\right) \\
& +\frac{G_{S^{3}}}{\hat{r}_{c}^{4}} \hat{L} \chi_{1}+\frac{\tilde{G}_{S^{3}}}{\hat{r}_{c}^{4}} \hat{L} \chi_{2},
\end{aligned}
$$

see Eqs. (B7a), and where $G_{S^{3}}$ and $\tilde{G}_{S^{3}}$ for aligned spins take the simple form

$$
\begin{aligned}
& G_{S^{3}}=\frac{1}{\hat{r}_{c}}\left(c_{n} \chi_{1}^{2}+c_{n} \chi_{1} \chi_{2}\right), \\
& \tilde{G}_{S^{3}}=\frac{1}{\hat{r}_{c}}\left(c_{n} \chi_{2}^{2}+c_{n} \chi_{1} \chi_{2}\right) .
\end{aligned}
$$

Including spin-cubic contributions was discussed in Appendix A of Ref. [47], which we implement here so that the effective Hamiltonian includes all PN information at the same order as the other Hamiltonians considered in this paper. Explicit results after matching at 4PN can be found in Appendix B 3.

\section{EFFECTIVE-ONE-BODY HAMILTONIAN WITH TEST-SPIN LIMIT: SEOB $_{\text {TS }}$}

The SEOB Hamiltonian proposed in Refs. [27,28] is based on the Hamiltonian of a spinning test body in the background of a Kerr black hole, which we here denote by 
SEOB $_{\mathrm{TS}}$ (see Table I). In this section, we extend that Hamiltonian to 4PN order; compared to previous results, we add $\mathrm{NLO} \mathrm{S}^{2}$, NNLO ${ }^{2} \mathrm{LO} \mathrm{S}^{3}$, and $\mathrm{LO} \mathrm{S}^{4} \mathrm{PN}$ corrections, for generic compact objects and spin orientations.

The $\mathrm{SEOB}_{\mathrm{TS}}$ Hamiltonian, as expressed in Ref. [32], is given by

$$
\begin{aligned}
\hat{H}^{\mathrm{eff}}= & \hat{H}_{\mathrm{even}}^{\mathrm{eff}}+\hat{H}_{\mathrm{odd}}^{\mathrm{eff}}+\hat{H}_{S_{*}}^{\mathrm{eff}}, \\
\hat{H}_{\mathrm{even}}^{\mathrm{eff}}= & \alpha \sqrt{q+Q^{0}}, \\
\hat{H}_{\mathrm{odd}}^{\mathrm{eff}}= & \beta \hat{p}_{\phi}, \\
\hat{H}_{S_{*}}^{\mathrm{eff}}= & {\left[\hat{\boldsymbol{F}}_{t}+\left(\beta+\frac{\alpha \gamma^{\phi \phi} \hat{p}_{\phi}}{\sqrt{q}}\right) \hat{\boldsymbol{F}}_{\phi}\right] \cdot \hat{\boldsymbol{S}}_{*} } \\
& +\frac{\alpha}{\sqrt{q}}\left(\gamma^{r r} \hat{p}_{r} \hat{\boldsymbol{F}}_{r}+\gamma^{\theta \theta} \hat{p}_{\theta} \hat{\boldsymbol{F}}_{\theta}\right) \cdot \hat{\boldsymbol{S}}_{*} \\
& +\frac{1}{2 \hat{r}^{3}}\left[3\left(\hat{\boldsymbol{S}}_{*} \cdot \boldsymbol{n}\right)^{2}-\hat{\boldsymbol{S}}_{*} \cdot \hat{\boldsymbol{S}}_{*}\right],
\end{aligned}
$$

where

$$
q=1+\gamma^{\phi \phi} \hat{p}_{\phi}^{2}+\gamma^{r r} \hat{p}_{r}^{2}+\gamma^{\theta \theta} \hat{p}_{\theta}^{2},
$$

and $\hat{\boldsymbol{S}}_{*} \equiv \boldsymbol{S}_{*} / M \mu$ is a rescaling of the spin of the test body. The spins are mapped according to

$$
\begin{aligned}
\boldsymbol{S}_{*} & =\boldsymbol{\sigma}_{*}\left[1+\nu f_{*}(r, \mathbf{p})\right]+\nu g_{*}(r, \mathbf{p}) \boldsymbol{\sigma}, \\
\boldsymbol{\sigma} & =\boldsymbol{S}_{1}+\boldsymbol{S}_{2}, \\
\boldsymbol{\sigma}_{*} & =\frac{m_{2}}{m_{1}} \boldsymbol{S}_{1}+\frac{m_{1}}{m_{2}} \boldsymbol{S}_{2},
\end{aligned}
$$

where the functions $f_{*}$ and $g_{*}$ are given by Eqs. (50)-(52) of Ref. [28], and that $\sigma=M \boldsymbol{a}$ is the spin of the background Kerr metric; it does not hold $\boldsymbol{a}=\boldsymbol{a}_{1}+\boldsymbol{a}_{2}$ as for the models discussed above. The spin maps are analogous to the mapping of the masses $M, \mu$ according to Eq. (1), with the difference that the spin maps relate dynamical variables. The deformed metric is obtained by substituting $\Delta, \Sigma$, and $\Lambda$ in the Kerr metric by

$$
\begin{aligned}
& \Delta_{t}=\hat{r}^{2} A(r)+\hat{\sigma}^{2}, \\
& \Delta_{r}=\Delta_{t} D^{0}(r), \\
& \hat{\Sigma}=\hat{r}^{2}+\hat{\sigma}^{2} \cos ^{2} \theta, \\
& \Lambda_{t}=\left(\hat{r}^{2}+\hat{\sigma}^{2}\right)^{2}-\hat{\sigma}^{2} \Delta_{t} \sin ^{2} \theta,
\end{aligned}
$$

as in

$$
\begin{array}{rlrl}
\alpha & =\frac{\sqrt{\Delta_{t} \hat{\Sigma}}}{\sqrt{\Lambda_{t}}}, \quad \beta=\frac{2 \hat{\sigma} \hat{r}}{\Lambda_{t}}, \\
\gamma^{\phi \phi} & =\frac{\hat{\Sigma}}{\Lambda_{t} \sin ^{2} \theta}, & \gamma^{r r}=\frac{\Delta_{r}}{\hat{\Sigma}}, & \gamma^{\theta \theta}=\frac{1}{\hat{\Sigma}} .
\end{array}
$$

The potential $D^{0}(r)$ is given by Eq. (28b), and the potential $A$ is given by

$$
A=\hat{a}^{2}\left(\frac{1}{\hat{r}}-\frac{1}{\hat{r}_{H,+}}\right)\left(\frac{1}{\hat{r}}-\frac{1}{\hat{r}_{H,-}}\right) A^{0}(r)-\frac{\hat{a}^{2}}{\hat{r}^{2}},
$$

where $A^{0}$ is given by Eq. (28a), and $\hat{r}_{H, \pm}$ are the scaled inner and outer radii of a Kerr black hole, i.e.,

$$
\hat{r}_{H, \pm}=1 \pm \sqrt{1-\hat{a}^{2}} .
$$

Finally, the vectors $\hat{\boldsymbol{F}}_{t}, \hat{\boldsymbol{F}}_{r}, \hat{\boldsymbol{F}}_{\theta}$, and $\hat{\boldsymbol{F}}_{\phi}$ describe the fictitious force acting on the test-body spin $\hat{\boldsymbol{S}}_{*}$ (frame dragging) in the deformed Kerr metric. They are given by Eq. (6) in Ref. [32], which we rewrite here for convenience,

$$
\begin{aligned}
& \hat{\boldsymbol{F}}_{\phi}=\cos \theta \hat{\boldsymbol{n}}+\hat{\boldsymbol{v}}, \\
& \hat{\boldsymbol{F}}_{t}=\hat{\boldsymbol{n}} \frac{\sqrt{\gamma^{\phi \phi}} \sqrt{\gamma^{\theta \theta}}}{\sqrt{q}}\left[\frac{\hat{p}_{\phi} \alpha_{, \theta}(1+2 \sqrt{q})}{(1+\sqrt{q})}-\alpha \hat{p}_{\phi} \cot \theta-\frac{(1-2 \sqrt{q}) \beta_{, \theta}}{2 \gamma^{\phi \phi}}\right], \\
& +\hat{\boldsymbol{v}} \frac{\csc \theta \sqrt{\gamma^{r r}}}{\sqrt{\gamma^{\phi \phi}}}\left[\frac{\gamma^{\phi \phi} \hat{p}_{\phi} \alpha_{, r}}{(1+\sqrt{q})}+\frac{(2 \sqrt{q}-1) \beta_{, r}+\alpha \hat{p}_{\phi} \gamma_{, r}^{\phi \phi}}{2 \sqrt{q}}\right] \text {, } \\
& \hat{\boldsymbol{F}}_{r}=-\hat{\boldsymbol{n}} \frac{\sqrt{\gamma^{\theta \theta}}\left(\beta_{, \theta} \hat{p}_{r}+\beta_{, r} \hat{p}_{\theta}\right)}{2 \alpha \sqrt{\gamma^{\phi \phi}}(1+\sqrt{q})}-\hat{\boldsymbol{v}} \frac{\csc \theta\left(\beta_{, \theta} \gamma^{\theta \theta} \hat{p}_{\theta}+2 \hat{p}_{r} \gamma^{r r} \beta_{, r}\right)}{2 \alpha \sqrt{\gamma^{\phi \phi}} \sqrt{\gamma^{r r}}(1+\sqrt{q})}-\hat{\boldsymbol{\xi}} \frac{\csc \theta \sqrt{\gamma^{\theta \theta}}}{2 \alpha \sqrt{\gamma^{r r}}}\left[\frac{2 \sqrt{q} \alpha_{, \theta}+\hat{p}_{\phi} \beta_{, \theta}}{(1+\sqrt{q})}+\frac{\alpha \gamma_{, \theta}^{\theta \theta}}{\gamma^{\theta \theta}}\right], \\
& \hat{\boldsymbol{F}}_{\theta}=-\hat{\boldsymbol{n}} \frac{\sqrt{\gamma^{\theta \theta}} \beta_{, \theta} \hat{p}_{\theta}}{\alpha \sqrt{\gamma^{\phi \phi}}(1+\sqrt{q})}-\hat{\boldsymbol{v}} \frac{\csc \theta \sqrt{\gamma^{r r}} \hat{p}_{\theta} \beta_{, r}}{2 \alpha \sqrt{\gamma^{\phi \phi}}(1+\sqrt{q})}+\hat{\boldsymbol{\xi}} \csc \theta\left[1+\frac{\sqrt{\gamma^{r r}}}{2 \alpha \sqrt{\gamma^{\theta \theta}}}\left(\frac{2 \sqrt{q} \alpha_{, r}+\hat{p}_{\phi} \beta_{, r}}{(1+\sqrt{q})}+\frac{\alpha \gamma_{, r}^{\theta \theta}}{\gamma^{\theta \theta}}\right)\right] .
\end{aligned}
$$


Here, the unit vectors $(\hat{\boldsymbol{n}}, \hat{\boldsymbol{\xi}}, \hat{\boldsymbol{v}})$ are defined by $\hat{\boldsymbol{n}}=\frac{x}{r}$, $\hat{\boldsymbol{\xi}}=\hat{e}_{\mathrm{Z}}^{\sigma} \times \hat{\boldsymbol{n}}$, and $\hat{\boldsymbol{v}}=\hat{\boldsymbol{n}} \times \hat{\boldsymbol{\xi}}$, where $\hat{e}_{\mathrm{Z}}^{\sigma}=\boldsymbol{\sigma} / \sigma$ denotes the direction of the (deformed) Kerr spin.

For the purpose of extending the $\mathrm{SEOB}_{\mathrm{TS}}$ Hamiltonian to $4 \mathrm{PN}$ in the spinning sector, we deviate from the original philosophy of Refs. [27,28] in that we do not modify the spin maps or deform the metric entering $H_{S_{*}}^{\text {eff }}$ with terms of quadratic and higher order in spin. Instead, we only slightly modify the Ansatz for the effective Hamiltonian (keeping $H_{S_{*}}^{\text {eff }}$ unchanged) as

$$
\begin{aligned}
& \hat{H}_{\mathrm{even}}^{\mathrm{eff}}=\sqrt{\alpha^{2}+A^{S S}+A^{n S}+A^{S^{4}}} \sqrt{q+B_{p}^{n S} \hat{\boldsymbol{p}}^{2}+\left(B_{n p}^{S S}+B_{n p}^{n S}\right) \hat{p}_{r}^{2}+Q^{0}+Q^{S^{2}}+Q^{S^{4}}}, \\
& \hat{H}_{\mathrm{odd}}^{\mathrm{eff}}=\beta \hat{p}_{\phi}+\frac{G_{S^{3}}}{\hat{r}^{4}} \hat{\boldsymbol{L}} \cdot \chi_{1}+\frac{\tilde{G}_{S^{3}}}{\hat{r}^{4}} \hat{\boldsymbol{L}} \cdot \chi_{2},
\end{aligned}
$$

where we introduced potentials into $H_{\mathrm{even}}^{\mathrm{eff}}$ at quadratic and higher order in spin following the structure of Eq. (19). These potentials $A^{S S}, A^{n S}, B_{p}^{n S}, B_{n p}^{S S}, B_{n p}^{n S}, Q^{S^{2}}$, and $A^{S^{4}}$ are given by Eqs. (29) and (30) but with $r$ instead of $r_{c}$ (and different $c_{n}$ ), and similarly for the spin-cubic corrections $G_{S^{3}}$ and $\tilde{G}_{S^{3}}$ from Eq. (32b). We take the function $Q^{S^{4}}$ to have the form

$$
\begin{aligned}
Q^{S^{4}}= & \frac{\hat{p}_{r}^{2}}{\hat{r}^{4}}\left[c_{n}\left(\boldsymbol{n} \cdot \chi_{1}\right)^{3}\left(\boldsymbol{n} \cdot \chi_{2}\right)+c_{n}\left(\boldsymbol{n} \cdot \chi_{1}\right)^{2}\left(\chi_{1} \cdot \chi_{2}\right)+c_{n}\left(\chi_{1} \cdot \chi_{1}\right)\left(\boldsymbol{n} \cdot \chi_{1}\right)\left(\boldsymbol{n} \cdot \chi_{2}\right)+c_{n}\left(\boldsymbol{n} \cdot \chi_{2}\right)^{3}\left(\boldsymbol{n} \cdot \chi_{1}\right)\right. \\
& +c_{n}\left(\boldsymbol{n} \cdot \chi_{2}\right)^{2}\left(\chi_{1} \cdot \chi_{2}\right)+c_{n}\left(\chi_{2} \cdot \chi_{2}\right)\left(\boldsymbol{n} \cdot \chi_{1}\right)\left(\boldsymbol{n} \cdot \chi_{2}\right)+c_{n}\left(\boldsymbol{n} \cdot \chi_{1}\right)^{2}\left(\boldsymbol{n} \cdot \chi_{2}\right)^{2}+c_{n}\left(\boldsymbol{n} \cdot \chi_{1}\right)^{2}\left(\chi_{2} \cdot \chi_{2}\right) \\
& \left.+c_{n}\left(\chi_{1} \cdot \chi_{1}\right)\left(\chi_{2} \cdot \chi_{2}\right)+c_{n}\left(\chi_{1} \cdot \chi_{1}\right)\left(\boldsymbol{n} \cdot \chi_{2}\right)^{2}\right] \\
& +\boldsymbol{p} \cdot \chi_{1} \frac{\hat{p}_{r}}{\hat{r}^{4}}\left[c_{n}\left(\chi_{2} \cdot \chi_{2}\right)\left(\boldsymbol{n} \cdot \chi_{2}\right)+c_{n}\left(\boldsymbol{n} \cdot \chi_{2}\right)^{3}+c_{n}\left(\boldsymbol{n} \cdot \chi_{1}\right)^{2}\left(\boldsymbol{n} \cdot \chi_{2}\right)+c_{n}\left(\boldsymbol{n} \cdot \chi_{1}\right)\left(\chi_{1} \cdot \chi_{2}\right)+c_{n}\left(\boldsymbol{n} \cdot \chi_{1}\right)\left(\boldsymbol{n} \cdot \chi_{2}\right)^{2}\right. \\
& \left.+c_{n}\left(\boldsymbol{n} \cdot \chi_{1}\right)\left(\chi_{2} \cdot \chi_{2}\right)\right] \\
& +\boldsymbol{p} \cdot \chi_{2} \frac{\hat{p}_{r}}{\hat{r}^{4}}\left[c_{n}\left(\chi_{1} \cdot \chi_{1}\right)\left(\boldsymbol{n} \cdot \chi_{1}\right)+c_{n}\left(\boldsymbol{n} \cdot \chi_{1}\right)^{3}+c_{n}\left(\boldsymbol{n} \cdot \chi_{2}\right)^{2}\left(\boldsymbol{n} \cdot \chi_{1}\right)+c_{n}\left(\boldsymbol{n} \cdot \chi_{2}\right)\left(\chi_{1} \cdot \chi_{2}\right)+c_{n}\left(\boldsymbol{n} \cdot \chi_{2}\right)\left(\boldsymbol{n} \cdot \chi_{1}\right)^{2}\right. \\
& \left.+c_{n}\left(\boldsymbol{n} \cdot \chi_{2}\right)\left(\chi_{1} \cdot \chi_{1}\right)\right] .
\end{aligned}
$$

Out of 38 possible terms in the most general expression for $Q^{S^{4}}, 16$ terms were removed via a gauge choice. We started by removing the three terms that do not vanish for aligned spins, but the other 13 terms were chosen arbitrarily. Explicit results after matching at $4 \mathrm{PN}$ can be found in Appendix B 4.

\section{COMPARISON WITH NUMERICAL RELATIVITY}

In this section, we compare the four SEOB Hamiltonians considered in this paper to NR simulations through the binding energy for circular orbits and aligned spins. The NR binding energy data we use here were extracted from the Simulating eXtreme Spacetimes (SXS) catalog [108] in Ref. [109]. Hereafter, in this section, we use the term aligned spins to mean spins parallel to, and in the same direction as, the orbital angular momentum $\boldsymbol{L}$, but we use the term "antialigned spins" to mean spins opposite to the direction of $\boldsymbol{L}$.

The binding energy is calculated by evaluating the EOB Hamiltonian for circular orbits $\left(p_{r}=0\right)$ and solving numerically $\dot{p}_{r}=-\partial H_{\mathrm{EOB}} / \partial r=0$ for the angular momentum $p_{\phi}$ at some radius. The orbital frequency $\Omega$ is obtained from

$$
\Omega=\frac{\partial H_{\mathrm{EOB}}}{\partial p_{\phi}}
$$

We then calculate the binding energy and orbital frequency as $r$ goes from the beginning of the NR simulation to the innermost-stable circular orbit (ISCO) of the Hamiltonian, which marks the end of the inspiral phase of the binary coalescence and the beginning of the plunge. The ISCO is calculated by setting both the first and second derivatives of the Hamiltonian with respect to $r$ to zero, i.e., $\partial H_{\mathrm{EOB}} / \partial r=0=\partial^{2} H_{\mathrm{EOB}} / \partial r^{2}$.

It should be noted that the binding energy is extracted from NR simulations from an evolving binary, tracking the radiated energy in GWs. From the EOB Hamiltonians, however, we obtain the binding energy here by assuming exact circular orbits at different orbital separations, neglecting the orbital decay (radiation-reaction) due to the emitted GWs. The NR and EOB binding energies are thus not expected to agree exactly here during the last few orbits (see discussions in Ref. [45]).

In Fig. 1, we plot the binding energy for nonspinning configurations with different mass ratios $q$ as a function of the velocity parameter $v \equiv(M \Omega)^{1 / 3}$, and we see that the 

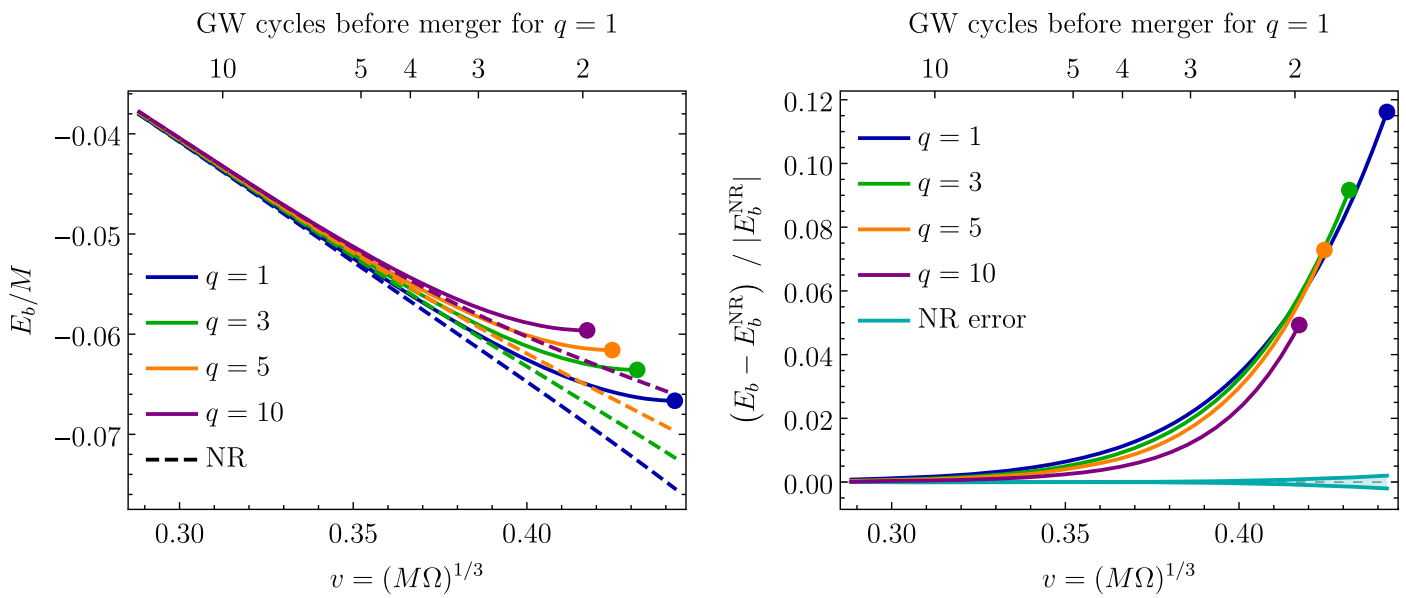

FIG. 1. Binding energy (left panel) and fractional binding energy (right panel) versus the "velocity" parameter $v$ for nonspinning binary-black-hole configurations with different mass ratios. The four SEOB Hamiltonians considered here are identical for zero spin. The relative NR error shown in the right panel is a conservative $1.1 \%$ estimate. The initial value of $v$ (the left end of the plots' domain) here is determined by the beginning of the NR simulation with $q=10$; those with lower mass ratios have several cycles at lower frequencies not shown here. We stress that the SEOB Hamiltonians at 4PN order are not calibrated to NR simulations.

binding energy increases with increasing mass ratio. The top axis of the figure indicates the number of GW cycles before merger, computed from the SXS waveform, for the case of $q=1$, which is close to the other values of $q$; for example, at two GW cycles before merger, $v=0.416$ for $q=1$ while $v=0.415$ for $q=10$. Since all SEOB Hamiltonians considered here agree in the nonspinning limit by construction, this figure gives a rough estimate for the zero-spin contributions to the binding energy. In all plots of this section, the number of GW cycles from merger is always computed from the SXS waveforms, and the merger is defined as the peak of the $(2,2)$ gravitational mode.

The different spin contributions to the binding energy are depicted in Fig. 2. They can be extracted by combining results for various spin combinations as (see Refs. [109,110])

$$
\begin{aligned}
E_{\mathrm{SO}}= & -\frac{1}{6}(-0.6,0)+\frac{8}{3}(0.3,0)-2(0,0)-\frac{1}{2}(0.6,0), \\
E_{S^{2}}= & \frac{3}{2}(-0.6,0)-2(0,0)+\frac{3}{2}(0.6,0)-(0.6,-0.6), \\
E_{S^{3}}= & -\frac{5}{6}(-0.6,0)-\frac{8}{3}(0.3,0)+3(0,0)-\frac{1}{2}(0.6,0) \\
& +\frac{1}{2}(0.6,-0.6)+\frac{1}{2}(0.6,0.6),
\end{aligned}
$$

where the numbers in brackets refer to the values of the dimensionless spins of the two bodies $\left(\chi_{1}, \chi_{2}\right)$. The spinsquared contributions to the binding energy $E_{S^{2}}$ refer to both $S_{i}^{2}$ and $S_{1} S_{2}$ interactions. Similarly, spin-cubic contributions $E_{S^{3}}$ refer to both $S_{i}^{3}$ and $S_{i}^{2} S_{j}$. We see that the spin-orbit contribution is about an order of magnitude larger than the
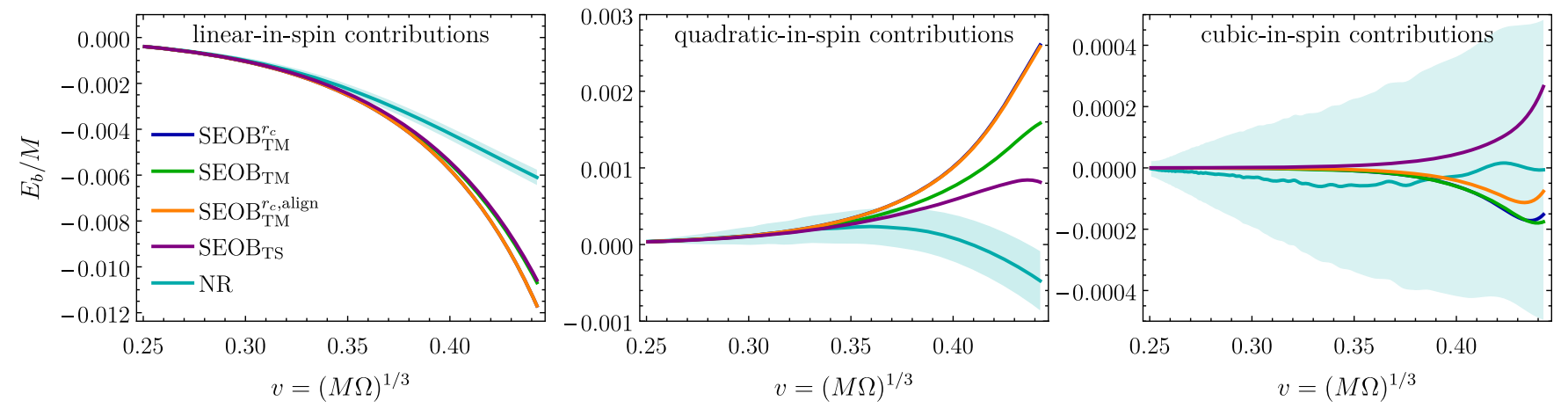

FIG. 2. Binding energy versus the velocity parameter $v$ for the linear-in-spin (left panel), quadratic-in-spin (central panel), and cubicin-spin (right panel) contributions of the four SEOB Hamiltonians. The NR error is indicated by the shaded regions. In the left panel, the blue and orange curves overlap since the $\mathrm{SEOB}_{\mathrm{TM}}^{r_{c}}$ and $\mathrm{SEOB}_{\mathrm{TM}}^{r_{c} \text {,align }}$ Hamiltonians are identical in the spin-orbit limit. 

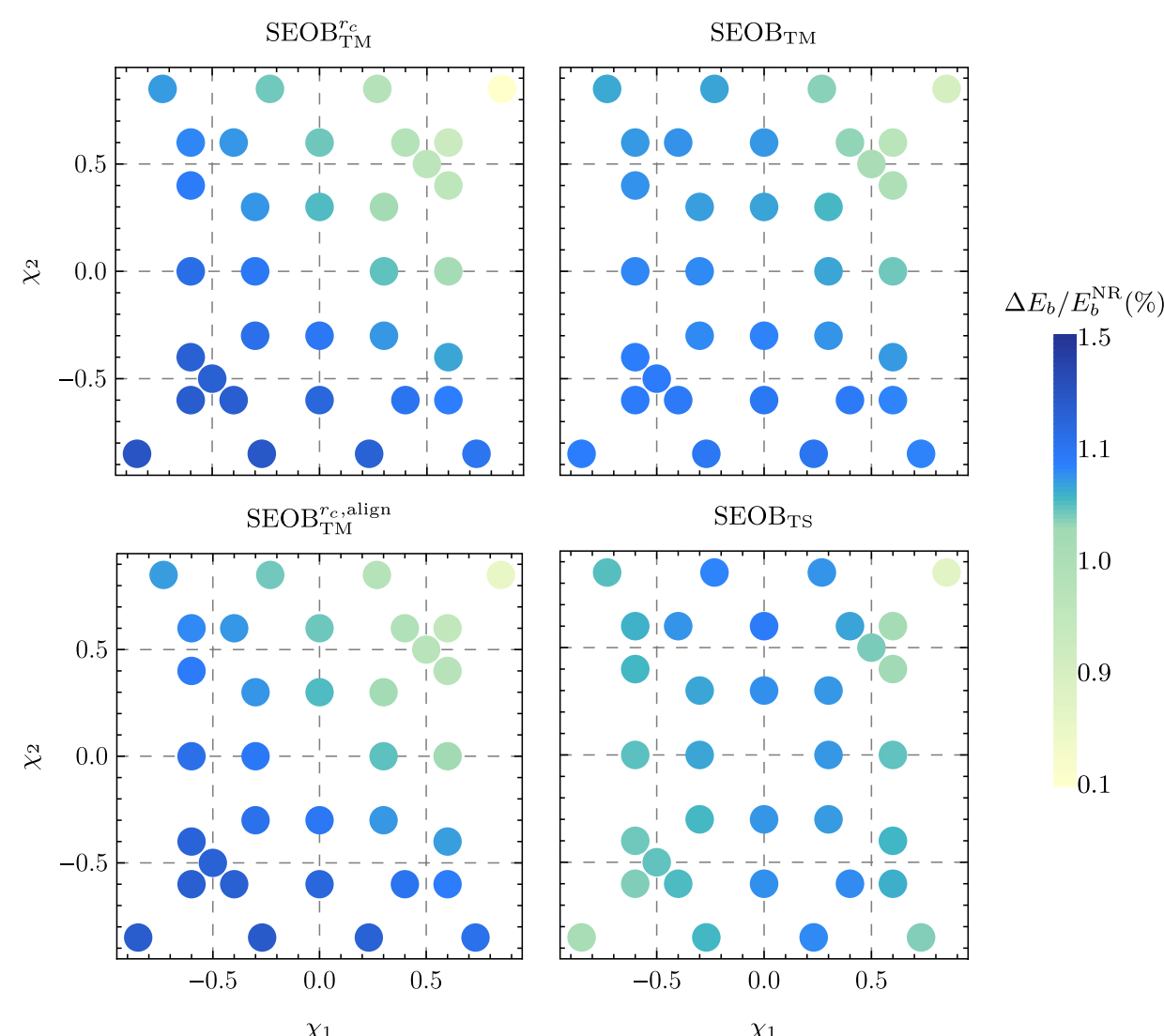

1.0

0.9

0.1

FIG. 3. Fractional difference in the binding energy between NR and SEOB Hamiltonians for different spin configurations with mass ratio $q=3$ at four $\mathrm{GW}$ cycles before merger.
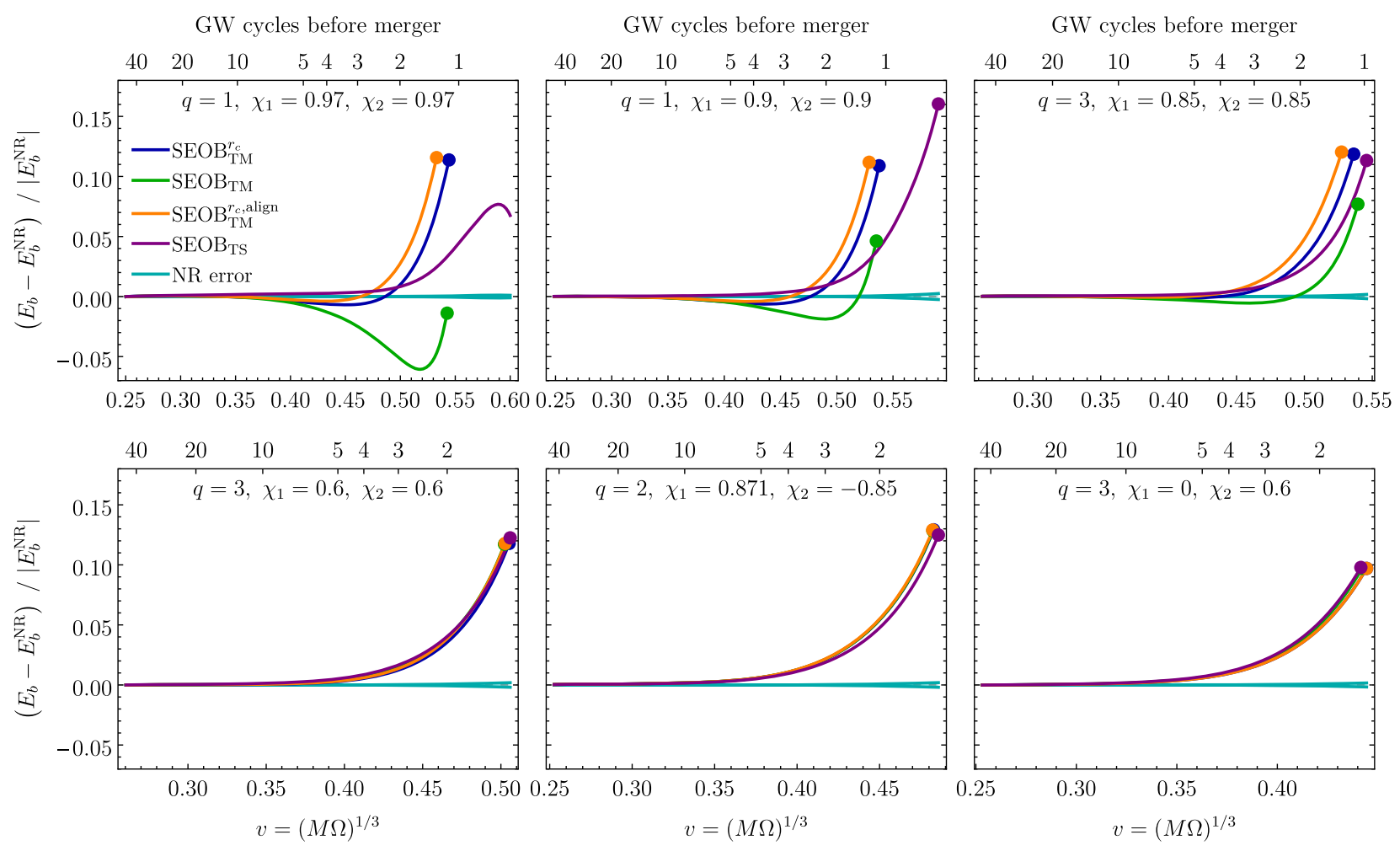

FIG. 4. Binding energy comparison with NR for different aligned-spin configurations for the four SEOB Hamiltonians. Curves that end with a point indicate the location of the ISCO. For the NR error, we used $1.1 \%$ relative error as a very conservative estimate. 


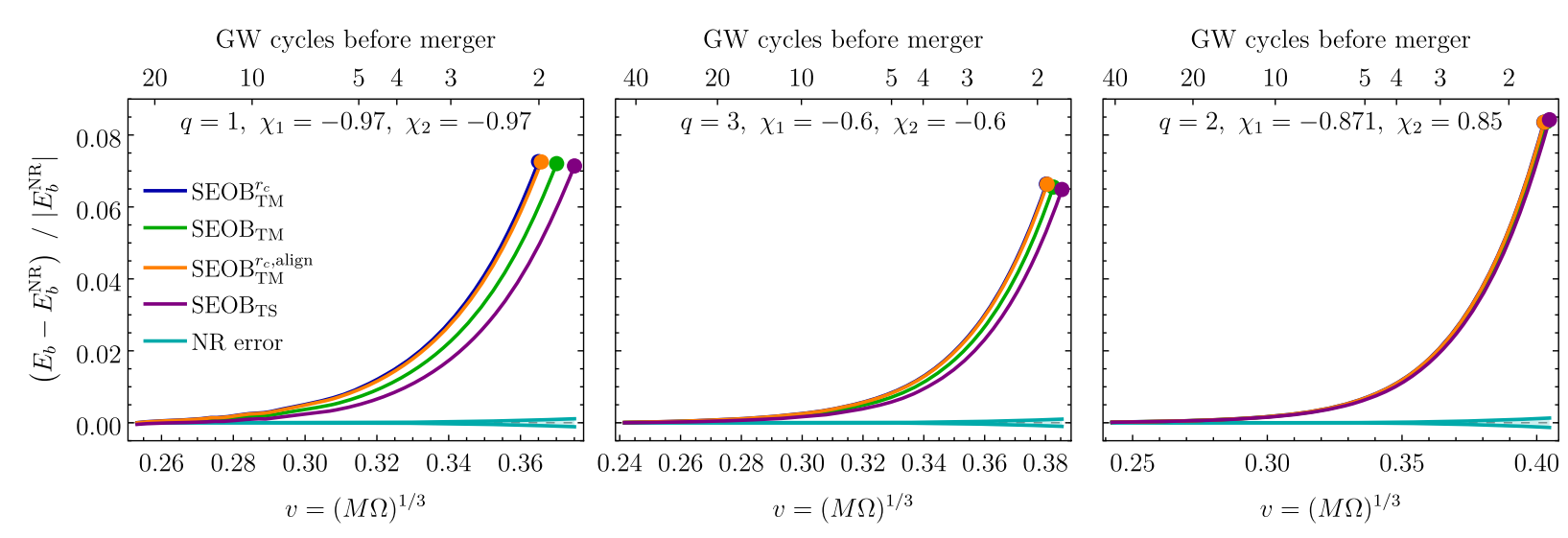

FIG. 5. As in Fig. 4 but for configurations with antialigned spins.

spin-squared contribution, which in turn is an order of magnitude larger than the spin-cubic contribution. All SEOB Hamiltonians give comparable results for the spinorbit part; however, for the spin-squared contribution, the $\mathrm{SEOB}_{\mathrm{TS}}$ and $\mathrm{SEOB}_{\mathrm{TM}}$ Hamiltonians give better agreement with NR than the other two Hamiltonians. For the cubic-inspin contributions, the NR error is larger than the EOB values for the binding energy, and hence we cannot conclude which Hamiltonian is better in terms of $\mathrm{S}^{3}$ contributions.

In Fig. 3, we compare the fractional energy difference $\left|E_{b}-E_{b}^{\mathrm{NR}}\right| / E_{b}^{\mathrm{NR}}$ at four $\mathrm{GW}$ cycles (i.e., two orbits) before merger for various spin configurations with mass ratio $q=3$. We see that, for all configurations at that frequency, the relative difference with NR is around $1 \%$. For aligned spins, all Hamiltonians give comparable results, but the $\mathrm{SEOB}_{\mathrm{TS}}$ Hamiltonian gives better agreement with NR for antialigned spins.

We also compare the binding energy as a function of velocity for some configurations with aligned spins (Fig. 4) and antialigned spins (Fig. 5). The curves in these figures start at the beginning of the available NR simulations and end at the ISCO of the EOB Hamiltonians. All effective Hamiltonians considered here have an ISCO for arbitrary spins, except that the $\mathrm{SEOB}_{\mathrm{TS}}$ Hamiltonian does not have an
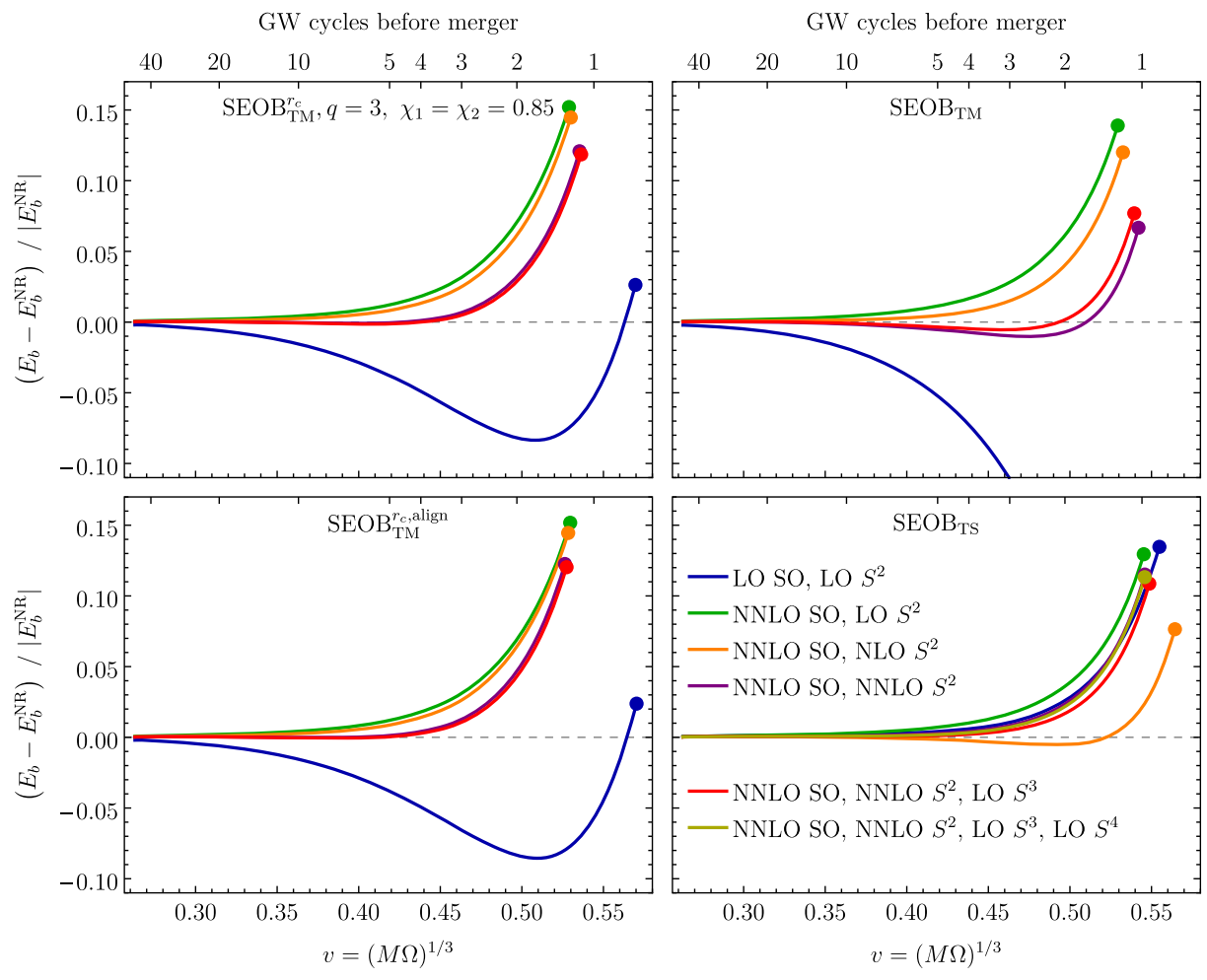

FIG. 6. Comparing the effect of adding spin contributions to the binding energy at different PN orders for the four SEOB Hamiltonians. 
ISCO for large aligned spins $\gtrsim 0.92$. From the three panels at the top of Fig. 4, we see that for large aligned spins ( $\gtrsim 0.8)$, the $\mathrm{SEOB}_{\mathrm{TS}}$ Hamiltonian shows slightly better agreement with NR than the other Hamiltonians. However, for smaller spins $(\lesssim 0.6)$, all Hamiltonians give very similar results. This is also the case when the two spins are both large but in opposite directions. For antialigned spins, the difference between the four Hamiltonians is smaller than in the alignedspin case; the $\mathrm{SEOB}_{\mathrm{TS}}$ Hamiltonian gives better agreement with NR than the other Hamiltonians, but the difference is small, even for spin magnitudes of 0.97 , and becomes negligible for smaller spins.

Finally, in Fig. 6, we compare the effect of spin PN orders to the effective Hamiltonian for a configuration with mass ratio $q=3$ and spins $\chi_{1}=\chi_{2}=0.85$. For all Hamiltonians, adding higher spin orders improves agreement with NR, except for the $\mathrm{SEOB}_{\mathrm{TS}}$ Hamiltonian where adding $\mathrm{LO} \mathrm{S}^{3}$ and $\mathrm{LO} \mathrm{S}^{4}$ gives slightly worse agreement. We checked that using different spin configurations gives qualitatively similar behavior.

Overall, beside the small differences pointed out above, all Hamiltonians perform reasonably well compared to NR simulations. One should expect that the differences that accumulate during the last orbits can be compensated by a calibration of the Hamiltonians, applying also further resummations to the potentials, which we leave for future work. This would be of particular interest for the simplified Hamiltonian, in order to prepare and evaluate it as a possible starting point for an EOB waveform model.

\section{CONCLUSIONS}

In this paper, we built spinning EOB Hamiltonians that include the complete fourth post-Newtonian conservative dynamics for generic (precessing) spins. These Hamiltonians are also valid for generic compact objects (e.g., black holes or neutron stars) since we included multipole constants that parametrize the deformation of the compact object due to its rotation.

In particular, we considered and extended four SEOB Hamiltonians: (i) an extension of the SEOB Hamiltonian from Ref. [17] by adding NNLO $\mathrm{S}^{2}$ and LO $\mathrm{S}^{3}$ contributions, in addition to adding the multipole constants; (ii) a simplified version of that Hamiltonian that differs in how the spin corrections are added to the Kerr metric, and that does not use the concept of centrifugal radius; (iii) the aligned-spin Hamiltonian from Refs. [19,23,47], which already includes complete 4PN information for generic compact objects, but considered here for comparison with the other Hamiltonians; (iv) an extension of the SEOB Hamiltonian from Refs. [27,28], which uses a test spin, by adding NLO $\mathrm{S}^{2}$, NNLO S${ }^{2}$, LO $\mathrm{S}^{3}$, and $\mathrm{LO} \mathrm{S}^{4}$ contributions, in addition to adding the multipole constants. Since our goal in this paper was to improve the description of spin effects in the EOB formalism, we modified the zero-spin part of the above Hamiltonians such that they are identical in that limit. Furthermore, we did not include NR calibration parameters or resummations of the PN corrections (e.g., with Padé or log resummations of the zero-spin part) since they constitute an implicit calibration to NR that improves the performance of EOB Hamiltonians only in certain models.

We compared the four SEOB Hamiltonians considered here with NR simulations by calculating the binding energy for circular orbits and aligned spins. We found that all Hamiltonians show good agreement with NR, and that the difference between the Hamiltonians is quite small up to moderate values of the spins and a handful number of GW cycles before merger. For large spins, the $\mathrm{SEOB}_{\mathrm{TS}}$ Hamiltonian performs better at large frequencies, but since all Hamiltonians have an error of about $1 \%$ compared to NR at about four GW cycles before merger, the simplest SEOB Hamiltonian SEOB $_{\mathrm{TM}}$ could be an excellent candidate for building an improved EOB waveform model with precessing spins. The simplicity will allow one to have a fast-to-evolve set of equations of motion and could help in calibrating the EOB waveforms built with $\mathrm{SEOB}_{\mathrm{TM}}$ to NR simulations. However, more analyses, which include dissipative effects, and a careful study of how the GW frequency approaches merger, are needed to pin down the more suitable SEOB Hamiltonian. Indeed, as several studies have shown [33-38], to attach robustly the mergerringdown waveform to the inspiral-plunge one in the EOB formalism, dynamical quantities, such as the orbital frequency, radial separation, and momentum vectors, have to behave regularly around and beyond the EOB photon orbit.

We leave for future work to complete the SEOB Hamiltonians to a gravitational waveform model, i.e., provide resummed expressions for GW modes and associated radiation-reaction forces. Once radiation-reaction forces are included in the model, it is important to perform comparisons with NR for precessing spins and to use those comparisons to study different resummation options and to add calibration parameters in order to improve the accuracy of EOB waveforms toward merger.

\section{ACKNOWLEDGMENTS}

We thank Sergei Ossokine for providing us with the numerical-relativity data for the binding energy used in this paper.

\section{APPENDIX A: COMPLETING THE SPINNING EFFECTIVE-ONE-BODY HAMILTONIAN IN REF. [17] AT NLO S ${ }^{2}$}

We found that Ref. [17] missed a contribution in the matching between the EOB Hamiltonian and PN results, namely, from the LO spin-squared canonical transformation, generated by $G_{\mathrm{SS}}^{\mathrm{LO}}$, applied to the LO spin-orbit Hamiltonian $H_{\mathrm{SO}}^{\mathrm{LO}}$. This contribution can be obtained using the Poisson bracket as $\left\{G_{\mathrm{SS}}^{\mathrm{LO}}, H_{\mathrm{SO}}^{\mathrm{LO}}\right\}$. This leads to SS 
contributions via the Poisson bracket of the spin vector $\left\{S^{i}, S^{j}\right\}=\epsilon_{i j k} S^{k}$, which turns out to start at NLO in the SS sector. Taking these additional contributions into account, we find that the coefficients in the EOB potentials $a_{i j}^{\chi}$, $a_{i j}^{n \chi}$, $b_{i j}^{p, \chi}, b_{i j}^{p, n \chi}, b_{i j}^{n p, \chi}, b_{i j}^{n p, n \chi}$, defined in Ref. [17] should read, assuming the gauge conditions $b_{i j}^{p, \chi}=0$,

$$
\begin{aligned}
& a_{11}^{n \chi}=7 \nu X_{1}+\frac{5}{4} \nu^{2}, \\
& a_{22}^{n \chi}=7 \nu X_{2}+\frac{5}{4} \nu^{2}, \\
& a_{12}^{n \chi}=a_{21}^{n \chi}=\frac{27}{8} \nu-\frac{9}{4} \nu^{2}, \\
& b_{11}^{p, n \chi}=4 \nu X_{1}-\frac{5}{2} \nu^{2}, \\
& b_{22}^{p, n \chi}=4 \nu X_{2}-\frac{5}{2} \nu^{2}, \\
& b_{12}^{p, n \chi}=b_{21}^{p, n \chi}=\frac{9}{8} \nu+\nu^{2} \\
& b_{11}^{n p, \chi}=9 \nu X_{1}-\frac{15}{4} \nu^{2}, \\
& b_{22}^{n p, \chi}=9 \nu X_{2}-\frac{15}{4} \nu^{2}, \\
& b_{11}^{n p, \chi}=b_{21}^{n p, \chi}=3 \nu+\frac{9}{4} \nu^{2}, \\
& b_{11}^{n p, n \chi}=15 \nu X_{1}-\frac{15}{4} \nu^{2},
\end{aligned}
$$

$$
\begin{aligned}
& b_{22}^{n p, n \chi}=15 \nu X_{2}-\frac{15}{4} \nu^{2}, \\
& b_{12}^{n p, n \chi}=b_{21}^{n p, n \chi}=\frac{45}{8} \nu+\frac{15}{4} \nu^{2},
\end{aligned}
$$

modifying Eqs. (2.52a) $-(2.53 \mathrm{c})$ and (2.62) in Ref. [17]. The coefficients $a_{i j}^{\chi}$ from Eq. (2.61) in Ref. [17] remain unchanged. Also, the coefficients $b_{i j}^{n p, \chi}$ are unchanged, but their defining Eq. (2.62) in Ref. [17] is no longer valid, so we listed them here explicitly for clarity. This solution has the three additional terms with coefficients $b_{11}^{n p, n \chi}$, $b_{22}^{n p, n \chi}$, and $b_{12}^{n p, n \chi}$, which vanish in Ref. [17]. The six symmetries between the coefficients in Ref. [17] are also absent in our solution (assuming the gauge conditions $\left.b_{i j}^{p, \chi}=0\right)$.

\section{APPENDIX B: HAMILTONIAN COEFFICIENTS AFTER MATCHING TO PN RESULTS}

In this appendix, we present the results of matching the SEOB Hamiltonians using the procedure described in Sec. II B. Here, we express the multipole constants as

$\tilde{C}_{i\left(\mathrm{ES}^{2}\right)} \equiv C_{i\left(\mathrm{ES}^{2}\right)}-1, \quad \tilde{C}_{i\left(\mathrm{BS}^{2}\right)} \equiv C_{i\left(\mathrm{BS}^{2}\right)}-1, \quad$ etc.,

such that the black hole results are easily obtained by setting $\tilde{C}_{\ldots}=0$.

The expressions for the Hamiltonians and the potentials given in this appendix are provided as the Supplemental Material [105] in the form of Mathematica files.

\section{Coefficients of the SEOB ${ }_{\mathrm{TM}}^{r_{c}}$ Hamiltonian}

The spin-orbit and spin-cubed PN corrections in Eq. (31a) are given by

$$
\begin{aligned}
G_{S}= & 2\left[1+\frac{1}{\hat{r}_{c}} \frac{5 \nu}{16}+\frac{27 \nu}{16} \hat{p}_{r}^{2}+\frac{1}{\hat{r}_{c}^{2}}\left(\frac{41 \nu^{2}}{256}+\frac{51 \nu}{8}\right)+\frac{\hat{p}_{r}^{2}}{\hat{r}_{c}}\left(\frac{21 \nu}{4}-\frac{49 \nu^{2}}{128}\right)+\left(\frac{169 \nu^{2}}{256}-\frac{5 \nu}{16}\right) \hat{p}_{r}^{4}\right]^{-1}, \\
G_{S^{*}}= & \frac{3}{2}\left[1+\frac{1}{\hat{r}_{c}}\left(\frac{\nu}{2}+\frac{3}{4}\right)+\left(\frac{3 \nu}{2}+\frac{5}{4}\right) \hat{p}_{r}^{2}+\frac{\hat{p}_{r}^{2}}{\hat{r}_{c}}\left(-\frac{7 \nu^{2}}{8}+5 \nu-1\right)+\frac{1}{\hat{r}_{c}^{2}}\left(\frac{3 \nu^{2}}{8}+\frac{29 \nu}{4}+\frac{27}{16}\right)\right. \\
& \left.+\left(\frac{3 \nu^{2}}{8}+\frac{25 \nu}{12}+\frac{5}{48}\right) \hat{p}_{r}^{4}\right]^{-1},
\end{aligned}
$$




$$
\begin{aligned}
& G_{S^{3}}=\frac{1}{\hat{r}_{c}}\left\{\left(\boldsymbol{n} \cdot \chi_{1}\right)^{2}\left[\left(5 \nu+(5 \nu-5) X_{1}\right) \tilde{\boldsymbol{C}}_{1\left(\mathrm{BS}^{3}\right)}+\left(\frac{9 \nu X_{1}}{4}-\frac{9 \nu^{2}}{4}\right) \tilde{C}_{1\left(\mathrm{ES}^{2}\right)}-\frac{5 \nu^{2}}{2}+2 \nu+\left(\frac{9 \nu}{2}-2\right) X_{1}\right]\right. \\
& +\chi_{1}^{2}\left[\left((1-\nu) X_{1}-\nu\right) \tilde{C}_{1\left(\mathrm{BS}^{3}\right)}+\left(\frac{3 \nu^{2}}{4}-\frac{3 \nu X_{1}}{4}\right) \tilde{C}_{1\left(\mathrm{ES}^{2}\right)}-\frac{\nu^{2}}{4}+\frac{\nu X_{1}}{4}\right] \\
& +\boldsymbol{n} \cdot \chi_{1} \boldsymbol{n} \cdot \chi_{2}\left[\left(-6 \nu^{2}-\frac{15 \nu X_{1}}{2}\right) \tilde{C}_{1\left(\mathrm{ES}^{2}\right)}-\frac{5 \nu^{2}}{3}-\frac{2 \nu X_{1}}{3}\right] \\
& +\chi_{1} \cdot \chi_{2}\left[\left(\frac{3 \nu^{2}}{2}+\frac{3 \nu X_{1}}{2}\right) \tilde{C}_{1\left(\mathrm{ES}^{2}\right)}+\frac{4 \nu^{2}}{3}-\frac{5 \nu X_{1}}{12}\right]+\chi_{2}^{2}\left[\left(\frac{3 \nu X_{2}}{4}-\frac{3 \nu^{2}}{4}\right) \tilde{C}_{2\left(\mathrm{ES}^{2}\right)}-\frac{13 \nu^{2}}{12}+\frac{2 \nu X_{2}}{3}\right] \\
& \left.+\left(\boldsymbol{n} \cdot \chi_{2}\right)^{2}\left[\left(\frac{3 \nu^{2}}{4}-\frac{15 \nu X_{2}}{4}\right) \tilde{C}_{2\left(\mathrm{ES}^{2}\right)}+\frac{25 \nu^{2}}{6}-\frac{17 \nu X_{2}}{6}\right]\right\} \\
& +\frac{\hat{L}^{2}}{\hat{r}^{2}}\left[\left(\boldsymbol{n} \cdot \chi_{1}\right)^{2}\left(\frac{\nu^{2}}{2}-\frac{\nu X_{1}}{2}\right)+\boldsymbol{n} \cdot \chi_{1} \boldsymbol{n} \cdot \chi_{2}\left(\nu^{2}-\nu X_{1}\right)+\left(\boldsymbol{n} \cdot \chi_{2}\right)^{2}\left(\frac{\nu X_{2}}{2}-\frac{3 \nu^{2}}{2}\right)\right] \\
& +\hat{p}_{r}^{2}\left[\left(\boldsymbol{n} \cdot \chi_{1}\right)^{2}\left(\frac{5 \nu X_{1}}{2}-\frac{5 \nu^{2}}{2}\right)+\boldsymbol{n} \cdot \chi_{1} \boldsymbol{n} \cdot \chi_{2}\left(3 \nu X_{1}-\nu^{2}\right)+\left(\boldsymbol{n} \cdot \chi_{2}\right)^{2}\left(\frac{7 \nu^{2}}{2}-\frac{\nu X_{2}}{2}\right)\right] \text {, } \\
& \tilde{G}_{S^{3}}=G_{S^{3}} \text { with } 1 \leftrightarrow 2 .
\end{aligned}
$$

The spin-squared corrections in Eq. (29) are given by

$$
\begin{aligned}
& A^{S S}=\frac{1}{\hat{r}_{c}^{3}} \chi_{1}^{2}\left(\nu-X_{1}\right) \tilde{C}_{1\left(\mathrm{ES}^{2}\right)} \\
& +\frac{1}{\hat{r}_{c}^{4}}\left\{\chi_{1}^{2}\left[\left(6 \nu+(2 \nu-6) X_{1}\right) \tilde{C}_{1\left(\mathrm{ES}^{2}\right)}-\frac{\nu^{2}}{2}+3 \nu X_{1}\right]+\frac{1}{2} \chi_{1} \cdot \chi_{2}\left(2 \nu-\nu^{2}\right)\right\} \\
& +\frac{1}{\hat{r}_{c}^{5}}\left\{\chi_{1}^{2}\left[\left(-\frac{207 \nu^{2}}{28}+\frac{275 \nu}{14}+\left(\frac{533 \nu}{28}-\frac{275}{14}\right) X_{1}\right) \tilde{C}_{1\left(\mathrm{ES}^{2}\right)}+\frac{3 \nu^{3}}{8}-\frac{157 \nu^{2}}{8}+\left(\frac{123 \nu}{4}-\frac{45 \nu^{2}}{8}\right) X_{1}\right]\right. \\
& \left.+\frac{1}{2} \chi_{1} \cdot \chi_{2}\left(\frac{3 \nu^{3}}{4}+\frac{145 \nu^{2}}{8}+\frac{25 \nu}{2}\right)\right\}+1 \leftrightarrow 2 \\
& A^{n S}=\frac{1}{\hat{r}_{c}^{3}}\left(\boldsymbol{n} \cdot \chi_{1}\right)^{2}\left(3 X_{1}-3 \nu\right) \tilde{C}_{1\left(\mathrm{ES}^{2}\right)} \\
& +\frac{1}{\hat{r}_{c}^{4}}\left\{\left(\boldsymbol{n} \cdot \chi_{1}\right)^{2}\left[\left(-3 \nu^{2}-9 \nu+(9-3 \nu) X_{1}\right) \tilde{C}_{1\left(\mathrm{ES}^{2}\right)}-\frac{5 \nu^{2}}{4}-7 \nu X_{1}\right]+\frac{1}{2}\left(\boldsymbol{n} \cdot \chi_{2}\right)\left(\boldsymbol{n} \cdot \chi_{1}\right)\left(\frac{9 \nu^{2}}{2}-\frac{27 \nu}{4}\right)\right\} \\
& +\frac{1}{\hat{r}_{c}^{5}}\left\{( \boldsymbol { n } \cdot \chi _ { 1 } ) ^ { 2 } \left[\left(-\frac{7 \nu^{3}}{8}-\frac{641 \nu^{2}}{56}-\frac{150 \nu}{7}+\left(-\frac{47 \nu^{2}}{8}+\frac{22 \nu}{7}+\frac{150}{7}\right) X_{1}\right) \tilde{C}_{1\left(\mathrm{ES}^{2}\right)}+\frac{11 \nu^{3}}{4}-\frac{71 \nu^{2}}{12}\right.\right. \\
& \left.\left.+\left(-\frac{63 \nu^{2}}{4}-\frac{79 \nu}{3}\right) X_{1}\right]+\frac{1}{2}\left(\boldsymbol{n} \cdot \chi_{2}\right)\left(\boldsymbol{n} \cdot \chi_{1}\right)\left(\frac{3 \nu^{3}}{2}-\frac{265 \nu^{2}}{6}-\frac{387 \nu}{16}\right)\right\}+1 \leftrightarrow 2 \text {, } \\
& B_{p}^{n S}=\frac{1}{\hat{r}_{c}^{3}}\left\{\left(\boldsymbol{n} \cdot \chi_{1}\right)^{2}\left[\left(-3 \nu^{2}+3 \nu+(3 \nu-3) X_{1}\right) \tilde{C}_{1\left(\mathrm{ES}^{2}\right)}-\frac{5 \nu^{2}}{2}+4 \nu X_{1}\right]+\frac{1}{2} \boldsymbol{n} \cdot \chi_{1} \boldsymbol{n} \cdot \chi_{2}\left(2 \nu^{2}+\frac{9 \nu}{4}\right)\right\} \\
& +\frac{1}{\hat{r}_{c}^{4}}\left\{( \boldsymbol { n } \cdot \chi _ { 1 } ) ^ { 2 } \left[\left(-\frac{7 \nu^{3}}{8}-\frac{221 \nu^{2}}{8}+\frac{15 \nu}{2}+\left(-\frac{47 \nu^{2}}{8}+\frac{169 \nu}{4}-\frac{15}{2}\right) X_{1}\right) \tilde{C}_{1\left(\mathrm{ES}^{2}\right)}-\frac{889 \nu^{2}}{24}+\frac{27 \nu^{3}}{8}\right.\right. \\
& \left.\left.+\left(\frac{323 \nu}{12}-\frac{217 \nu^{2}}{8}\right) X_{1}\right]+\frac{1}{2} \boldsymbol{n} \cdot \chi_{1} \boldsymbol{n} \cdot \chi_{2}\left(\frac{11 \nu^{3}}{4}-\frac{427 \nu^{2}}{24}+\frac{57 \nu}{16}\right)\right\}+1 \leftrightarrow 2 \text {, }
\end{aligned}
$$




$$
\begin{aligned}
& B_{n p}^{n S}=\frac{1}{\hat{r}_{c}^{3}}\left\{\left(\boldsymbol{n} \cdot \chi_{1}\right)^{2}\left(\frac{15 \nu^{2}}{4}-15 \nu X_{1}\right)+\frac{1}{2} \boldsymbol{n} \cdot \chi_{1} \boldsymbol{n} \cdot \chi_{2}\left(-\frac{15 \nu^{2}}{2}-\frac{45 \nu}{4}\right)\right\} \\
& +\frac{1}{\hat{r}_{c}^{4}}\left\{( \boldsymbol { n } \cdot \chi _ { 1 } ) ^ { 2 } \left[\left(-\frac{7 \nu^{3}}{2}-\frac{\nu^{2}}{4}+\frac{9 \nu}{2}+\left(\frac{25 \nu^{2}}{2}+\frac{121 \nu}{4}-\frac{9}{2}\right) X_{1}\right) \tilde{C}_{1\left(\mathrm{ES}^{2}\right)}+\frac{17 \nu^{3}}{8}+\frac{185 \nu^{2}}{24}\right.\right. \\
& \left.\left.+\left(-\frac{23 \nu^{2}}{8}-\frac{619 \nu}{12}\right) X_{1}\right]+\frac{1}{2} \boldsymbol{n} \cdot \chi_{1} \boldsymbol{n} \cdot \chi_{2}\left(-\frac{47 \nu^{3}}{4}-\frac{4411 \nu^{2}}{24}-\frac{39 \nu}{2}\right)\right\}+1 \leftrightarrow 2, \\
& B_{n p}^{S S}=\frac{1}{\hat{r}_{c}^{3}}\left\{\chi_{1}^{2}\left[\left(-3 \nu^{2}+3 \nu+(3 \nu-3) X_{1}\right) \tilde{C}_{1\left(\mathrm{ES}^{2}\right)}-\frac{15 \nu^{2}}{4}+9 \nu X_{1}\right]+\frac{1}{2}\left(\frac{9 \nu^{2}}{2}+6 \nu\right) \chi_{1} \cdot \chi_{2}\right\} \\
& +\frac{1}{\hat{r}_{c}^{4}}\left\{\chi_{1}^{2}\left[\left(-\frac{159 \nu^{2}}{4}+\frac{23 \nu}{2}+\left(-12 \nu^{2}+\frac{197 \nu}{4}-\frac{23}{2}\right) X_{1}\right) \tilde{C}_{1\left(\mathrm{ES}^{2}\right)}+5 \nu^{3}-61 \nu^{2}+\left(\frac{275 \nu}{4}-37 \nu^{2}\right) X_{1}\right]\right. \\
& \left.+\frac{1}{2}\left(10 \nu^{3}+38 \nu^{2}+20 \nu\right) \chi_{1} \cdot \chi_{2}\right\}+1 \leftrightarrow 2 \\
& Q^{S^{2}}=\frac{\hat{p}_{r}^{3}}{\hat{r}_{c}^{3}}\left\{\boldsymbol{n} \cdot \chi_{1} \hat{\boldsymbol{p}} \cdot \chi_{1}\left[\left(20 \nu^{3}-35 \nu^{2}+\left(35 \nu-20 \nu^{2}\right) X_{1}\right) \tilde{\boldsymbol{C}}_{1\left(\mathrm{ES}^{2}\right)}+\frac{199 \nu^{3}}{8}-\frac{1085 \nu^{2}}{24}+\left(\frac{130 \nu}{3}-\frac{517 \nu^{2}}{8}\right) X_{1}\right]\right. \\
& \left.+\boldsymbol{n} \cdot \chi_{1} \hat{\boldsymbol{p}} \cdot \chi_{2}\left(-\frac{79 \nu^{3}}{8}+\frac{79 \nu^{2}}{12}+\frac{45 \nu}{16}\right)\right\} \\
& +\frac{\hat{p}_{r}^{4}}{\hat{r}_{c}^{3}}\left\{\chi_{1}^{2}\left[\left(5 \nu^{3}-\frac{35 \nu^{2}}{4}+\left(\frac{35 \nu}{4}-5 \nu^{2}\right) X_{1}\right) \tilde{C}_{1\left(\mathrm{ES}^{2}\right)}+\frac{55 \nu^{3}}{8}-\frac{105 \nu^{2}}{8}+\left(\frac{55 \nu}{4}-\frac{145 \nu^{2}}{8}\right) X_{1}\right]\right. \\
& +\left(\boldsymbol{n} \cdot \chi_{1}\right)^{2}\left[\left(\frac{245 \nu^{2}}{4}-35 \nu^{3}+\left(35 \nu^{2}-\frac{245 \nu}{4}\right) X_{1}\right) \tilde{C}_{1\left(\mathrm{ES}^{2}\right)}-\frac{91 \nu^{3}}{2}+\frac{1015 \nu^{2}}{12}+\left(119 \nu^{2}-\frac{1015 \nu}{12}\right) X_{1}\right] \\
& \left.+\frac{1}{2} \chi_{1} \cdot \chi_{2}\left(-\frac{25 \nu^{3}}{4}+\frac{45 \nu^{2}}{8}+\frac{5 \nu}{2}\right)+\frac{1}{2} \boldsymbol{n} \cdot \chi_{1} \boldsymbol{n} \cdot \chi_{2}\left(\frac{77 \nu^{3}}{2}-\frac{721 \nu^{2}}{24}-\frac{105 \nu}{8}\right)\right\}+1 \leftrightarrow 2 .
\end{aligned}
$$

The spin-quartic corrections in Eq. (30) are given by

$$
\begin{aligned}
& A^{S^{4}}=\frac{1}{\hat{r}_{c}^{5}}\left\{\left(\boldsymbol{n} \cdot \chi_{1}\right)^{4}\left[\left(\frac{21 \nu^{2}}{2}-\frac{21 \nu}{2}+\left(\frac{21}{2}-21 \nu\right) X_{1}\right) \tilde{C}_{1\left(\mathrm{ES}^{2}\right)}+\left(-\frac{35 \nu^{2}}{4}+\frac{35 \nu}{4}+\left(\frac{35 \nu}{2}-\frac{35}{4}\right) X_{1}\right) \tilde{C}_{1\left(\mathrm{ES}^{4}\right)}\right]\right. \\
& +\boldsymbol{n} \cdot \chi_{2}\left(\boldsymbol{n} \cdot \chi_{1}\right)^{3}\left[\left(35 \nu^{2}-35 \nu X_{1}\right) \tilde{C}_{1\left(\mathrm{BS}^{3}\right)}+\left(21 \nu X_{1}-21 \nu^{2}\right) \tilde{C}_{1\left(\mathrm{ES}^{2}\right)}\right] \\
& +\chi_{1} \cdot \chi_{2}\left(\boldsymbol{n} \cdot \chi_{1}\right)^{2}\left[\left(15 \nu X_{1}-15 \nu^{2}\right) \tilde{\boldsymbol{C}}_{1\left(\mathrm{BS}^{3}\right)}+\left(3 \nu^{2}-3 \nu X_{1}\right) \tilde{C}_{1\left(\mathrm{ES}^{2}\right)}\right] \\
& +\chi_{1}^{2}\left(\boldsymbol{n} \cdot \chi_{1}\right)^{2}\left[\left(-\frac{9 \nu^{2}}{2}+\frac{9 \nu}{2}+\left(9 \nu-\frac{9}{2}\right) X_{1}\right) \tilde{C}_{1\left(\mathrm{ES}^{2}\right)}+\left(\frac{15 \nu^{2}}{2}-\frac{15 \nu}{2}+\left(\frac{15}{2}-15 \nu\right) X_{1}\right) \tilde{\boldsymbol{C}}_{1\left(\mathrm{ES}^{4}\right)}\right] \\
& +\chi_{2}^{2}\left(\boldsymbol{n} \cdot \chi_{1}\right)^{2}\left[6 \tilde{C}_{2\left(\mathrm{ES}^{2}\right)} \nu^{2}+\tilde{C}_{1\left(\mathrm{ES}^{2}\right)}\left(\frac{15}{2} \tilde{C}_{2\left(\mathrm{ES}^{2}\right)} \nu^{2}+\frac{15 \nu^{2}}{2}\right)\right] \\
& +\frac{1}{2}\left(\boldsymbol{n} \cdot \chi_{2}\right)^{2}\left(\boldsymbol{n} \cdot \chi_{1}\right)^{2}\left[\tilde{\boldsymbol{C}}_{1\left(\mathrm{ES}^{2}\right)}\left(-\frac{1}{2} 105 \tilde{\boldsymbol{C}}_{2\left(\mathrm{ES}^{2}\right)} \nu^{2}-42 \nu^{2}\right)-42 \nu^{2} \tilde{\boldsymbol{C}}_{2\left(\mathrm{ES}^{2}\right)}\right] \\
& +\chi_{1}^{2} \boldsymbol{n} \cdot \chi_{2} \boldsymbol{n} \cdot \chi_{1}\left[\left(15 \nu X_{1}-15 \nu^{2}\right) \tilde{\boldsymbol{C}}_{1\left(\mathrm{BS}^{3}\right)}+\left(6 \nu^{2}-6 \nu X_{1}\right) \tilde{\boldsymbol{C}}_{1\left(\mathrm{ES}^{2}\right)}\right] \\
& +\frac{1}{2} \boldsymbol{n} \cdot \chi_{2} \chi_{1} \cdot \chi_{2} \boldsymbol{n} \cdot \chi_{1}\left[27 \tilde{C}_{2\left(\mathrm{ES}^{2}\right)} \nu^{2}+\tilde{C}_{1\left(\mathrm{ES}^{2}\right)}\left(30 \tilde{C}_{2\left(\mathrm{ES}^{2}\right)} \nu^{2}+27 \nu^{2}\right)\right] \\
& +\chi_{1} \cdot \chi_{2} \chi_{1}^{2}\left(3 \nu^{2}-3 \nu X_{1}\right) \tilde{C}_{1\left(\mathrm{BS}^{3}\right)}+\chi_{1}^{4}\left[-\frac{3 \nu^{2}}{4}+\frac{3 \nu}{4}+\left(\frac{3 \nu}{2}-\frac{3}{4}\right) X_{1}\right] \tilde{C}_{1\left(\mathrm{ES}^{4}\right)} \\
& +\frac{1}{2} \chi_{1}^{2} \chi_{2}^{2}\left[\tilde{C}_{1\left(\mathrm{ES}^{2}\right)}\left(-\frac{1}{2} 3 \tilde{C}_{2\left(\mathrm{ES}^{2}\right)} \nu^{2}-\frac{3 \nu^{2}}{2}\right)-\frac{3}{2} \nu^{2} \tilde{C}_{2\left(\mathrm{ES}^{2}\right)}\right] \\
& \left.+\frac{1}{2}\left(\chi_{1} \cdot \chi_{2}\right)^{2}\left[\tilde{C}_{1\left(\mathrm{ES}^{2}\right)}\left(-3 \tilde{C}_{2\left(\mathrm{ES}^{2}\right)} \nu^{2}-3 \nu^{2}\right)-3 \nu^{2} \tilde{C}_{2\left(\mathrm{ES}^{2}\right)}\right]\right\}+1 \leftrightarrow 2 .
\end{aligned}
$$




\section{Coefficients of the $\mathrm{SEOB}_{\mathrm{TM}}$ Hamiltonian}

The spin-orbit and spin-cubed PN corrections in Eq. (34a) are given by

$$
\begin{aligned}
G_{S}= & {\left[1-\frac{27}{16} \nu \hat{p}_{r}^{2}-\frac{5 \nu}{16 \hat{r}}+\left(\frac{35 \nu^{2}}{16}+\frac{5 \nu}{16}\right) \hat{p}_{r}^{4}+\frac{\hat{p}_{r}^{2}}{\hat{r}}\left(\frac{23 \nu^{2}}{16}-\frac{21 \nu}{4}\right)+\frac{1}{\hat{r}^{2}}\left(-\frac{\nu^{2}}{16}-\frac{51 \nu}{8}\right)\right], } \\
G_{S^{*}}= & \frac{3}{2}\left[1-\left(\frac{3 \nu}{2}+\frac{5}{4}\right) \hat{p}_{r}^{2}-\frac{1}{\hat{r}}\left(\frac{3}{4}+\frac{\nu}{2}\right)+\left(\frac{15 \nu^{2}}{8}+\frac{5 \nu}{3}+\frac{35}{24}\right) \hat{p}_{r}^{4}+\frac{\hat{p}_{r}^{2}}{\hat{r}}\left(\frac{19 \nu^{2}}{8}-\frac{3 \nu}{2}+\frac{23}{8}\right)+\frac{1}{\hat{r}^{2}}\left(-\frac{\nu^{2}}{8}-\frac{13 \nu}{2}-\frac{9}{8}\right)\right], \\
G_{S^{3}}= & \frac{1}{\hat{r}}\left\{\left(\boldsymbol{n} \cdot \chi_{1}\right)^{2}\left[\left(5 \nu+(5 \nu-5) X_{1}\right) \tilde{C}_{1\left(\mathrm{BS}^{3}\right)}+\left(\frac{9 \nu X_{1}}{4}-\frac{9 \nu^{2}}{4}\right) \tilde{C}_{1\left(\mathrm{ES}^{2}\right)}-2 \nu^{2}+2 \nu X_{1}\right]\right. \\
& +\chi_{1}^{2}\left[\left((1-\nu) X_{1}-\nu\right) \tilde{C}_{1\left(\mathrm{BS}^{3}\right)}+\left(\frac{3 \nu^{2}}{4}-\frac{3 \nu X_{1}}{4}\right) \tilde{C}_{1\left(\mathrm{ES}^{2}\right)}+\frac{\nu^{2}}{2}-\frac{\nu X_{1}}{2}\right] \\
& +\chi_{1} \cdot \chi_{2}\left[\left(\frac{3 \nu^{2}}{2}+\frac{3 \nu X_{1}}{2}\right) \tilde{C}_{1(\mathrm{ES})}-\frac{\nu^{2}}{6}-\frac{5 \nu X_{1}}{12}\right]+\chi_{2}^{2}\left[\left(\frac{3 \nu X_{2}}{4}-\frac{3 \nu^{2}}{4}\right) \tilde{C}_{2\left(\mathrm{ES}^{2}\right)}-\frac{\nu^{2}}{3}-\frac{\nu X_{2}}{12}\right] \\
& \left.+\boldsymbol{n} \cdot \chi_{1} \boldsymbol{n} \cdot \chi_{2}\left[\left(-6 \nu^{2}-\frac{15 \nu X_{1}}{2}\right) \tilde{C}_{1\left(\mathrm{ES}^{2}\right)}-\frac{8 \nu^{2}}{3}+\frac{10 \nu X_{1}}{3}\right]+\left(\boldsymbol{n} \cdot \chi_{2}\right)^{2}\left[\left(\frac{3 \nu^{2}}{4}-\frac{15 \nu X_{2}}{4}\right) \tilde{C}_{2\left(\mathrm{ES}^{2}\right)}+\frac{14 \nu^{2}}{3}-\frac{4 \nu X_{2}}{3}\right]\right\} \\
& +\frac{\hat{L}^{2}}{r^{2}}\left[\left(\boldsymbol{n} \cdot \chi_{1}\right)^{2}\left(\frac{\nu^{2}}{2}-\frac{\nu X_{1}}{2}\right)+\boldsymbol{n} \cdot \chi_{1} \boldsymbol{n} \cdot \chi_{2}\left(\nu^{2}-\nu X_{1}\right)+\left(\boldsymbol{n} \cdot \chi_{2}\right)^{2}\left(\frac{\nu X_{2}}{2}-\frac{3 \nu^{2}}{2}\right)\right] \\
& +\hat{p}_{r}^{2}\left[\left(\boldsymbol{n} \cdot \chi_{1}\right)^{2}\left(\frac{5 \nu X_{1}}{2}-\frac{5 \nu^{2}}{2}\right)+\boldsymbol{n} \cdot \chi_{1} \boldsymbol{n} \cdot \chi_{2}\left(3 \nu X_{1}-\nu^{2}\right)+\left(\boldsymbol{n} \cdot \chi_{2}\right)^{2}\left(\frac{7 \nu^{2}}{2}-\frac{\nu X_{2}}{2}\right)\right],
\end{aligned}
$$

$\tilde{G}_{S^{3}}=G_{S^{3}} \quad$ with $\quad 1 \leftrightarrow 2$.

The spin-squared and spin-quartic corrections in Eq.

$$
\begin{aligned}
A^{S S}= & \frac{1}{\hat{r}^{3}} \chi_{1}^{2}\left(\nu-X_{1}\right) \tilde{C}_{1\left(\mathrm{ES}^{2}\right)} \\
& +\frac{1}{\hat{r}^{4}}\left\{\chi_{1}^{2}\left[\left(4 \nu+(2 \nu-4) X_{1}\right) \tilde{C}_{1\left(\mathrm{ES}^{2}\right)}-\frac{\nu^{2}}{2}+3 \nu X_{1}\right]+\frac{1}{2} \chi_{1} \cdot \chi_{2}\left(2 \nu-\nu^{2}\right)\right\} \\
& +\frac{1}{\hat{r}^{5}}\left\{\chi_{1}^{2}\left[\left(-\frac{207 \nu^{2}}{28}+\frac{163 \nu}{14}+\left(\frac{421 \nu}{28}-\frac{163}{14}\right) X_{1}\right) \tilde{C}_{1\left(\mathrm{ES}^{2}\right)}+\frac{3 \nu^{3}}{8}-\frac{125 \nu^{2}}{8}+\left(\frac{87 \nu}{4}-\frac{45 \nu^{2}}{8}\right) X_{1}\right]\right. \\
& \left.+\frac{1}{2} \chi_{1} \cdot \chi_{2}\left(\frac{3 \nu^{3}}{4}+\frac{113 \nu^{2}}{8}+\frac{17 \nu}{2}\right)\right\}+1 \leftrightarrow 2, \\
A^{n S}= & \frac{1}{\hat{r}^{3}}\left(\boldsymbol{n} \cdot \chi_{1}\right)^{2}\left(3 X_{1}-3 \nu\right) \tilde{C}_{1\left(\mathrm{ES}^{2}\right)} \\
& +\frac{1}{\hat{r}^{4}}\left\{\left(\boldsymbol{n} \cdot \chi_{1}\right)^{2}\left[\left(-3 \nu^{2}-9 \nu+(9-3 \nu) X_{1}\right) \tilde{C}_{1\left(\mathrm{ES}^{2}\right)}-\frac{5 \nu^{2}}{4}-7 \nu X_{1}\right]+\frac{1}{2} \boldsymbol{n} \cdot \chi_{1} \boldsymbol{n} \cdot \chi_{2}\left(\frac{9 \nu^{2}}{2}-\frac{27 \nu}{4}\right)\right\} \\
& +\frac{1}{\hat{r}^{5}}\left\{( \boldsymbol { n } \cdot \chi _ { 1 } ) ^ { 2 } \left[\left(-\frac{7 \nu^{3}}{8}-\frac{641 \nu^{2}}{56}-\frac{150 \nu}{7}+\left(-\frac{47 \nu^{2}}{8}+\frac{22 \nu}{7}+\frac{150}{7}\right) X_{1}\right) \tilde{C}_{1\left(\mathrm{ES}^{2}\right)}+\frac{11 \nu^{3}}{4}-\frac{71 \nu^{2}}{12}\right.\right. \\
& \left.\left.+\left(-\frac{63 \nu^{2}}{4}-\frac{79 \nu}{3}\right) X_{1}\right]+\frac{1}{2} \boldsymbol{n} \cdot \chi_{1} \boldsymbol{n} \cdot \chi_{2}\left(\frac{3 \nu^{3}}{2}-\frac{265 \nu^{2}}{6}-\frac{387 \nu}{16}\right)\right\}+1 \leftrightarrow 2,
\end{aligned}
$$




$$
\begin{aligned}
& B_{p}^{n S}=\frac{1}{\hat{r}^{3}}\left\{\left(\boldsymbol{n} \cdot \chi_{1}\right)^{2}\left[\left(3 \nu^{2}-3 \nu+(3-3 \nu) X_{1}\right) \tilde{\boldsymbol{C}}_{1\left(\mathrm{ES}^{2}\right)}+\frac{5 \nu^{2}}{2}-4 \nu X_{1}\right]+\frac{1}{2} \boldsymbol{n} \cdot \chi_{1} \boldsymbol{n} \cdot \chi_{2}\left(-2 \nu^{2}-\frac{9 \nu}{4}\right)\right\} \\
& +\frac{1}{\hat{r}^{4}}\left\{( \boldsymbol { n } \cdot \chi _ { 1 } ) ^ { 2 } \left[\left(\frac{7 \nu^{3}}{8}+\frac{221 \nu^{2}}{8}-\frac{15 \nu}{2}+\left(\frac{47 \nu^{2}}{8}-\frac{169 \nu}{4}+\frac{15}{2}\right) X_{1}\right) \tilde{C}_{1\left(\mathrm{ES}^{2}\right)}-\frac{27 \nu^{3}}{8}+\frac{889 \nu^{2}}{24}\right.\right. \\
& \left.\left.+\left(\frac{217 \nu^{2}}{8}-\frac{323 \nu}{12}\right) X_{1}\right]+\frac{1}{2} \boldsymbol{n} \cdot \chi_{1} \boldsymbol{n} \cdot \chi_{2}\left(-\frac{11 \nu^{3}}{4}+\frac{427 \nu^{2}}{24}-\frac{57 \nu}{16}\right)\right\}+1 \leftrightarrow 2 \text {, } \\
& B_{n p}^{n S}=\frac{1}{\hat{r}^{3}}\left\{\left(\boldsymbol{n} \cdot \chi_{1}\right)^{2}\left(\frac{15 \nu^{2}}{4}-15 \nu X_{1}\right)+\frac{1}{2} \boldsymbol{n} \cdot \chi_{1} \boldsymbol{n} \cdot \chi_{2}\left(-\frac{15 \nu^{2}}{2}-\frac{45 \nu}{4}\right)\right\} \\
& +\frac{1}{\hat{r}^{4}}\left\{( \boldsymbol { n } \cdot \chi _ { 1 } ) ^ { 2 } \left[\left(-\frac{7 \nu^{3}}{2}-\frac{\nu^{2}}{4}+\frac{9 \nu}{2}+\left(\frac{25 \nu^{2}}{2}+\frac{121 \nu}{4}-\frac{9}{2}\right) X_{1}\right) \tilde{C}_{1\left(\mathrm{ES}^{2}\right)}+\frac{17 \nu^{3}}{8}+\frac{365 \nu^{2}}{24}\right.\right. \\
& \left.\left.+\left(-\frac{23 \nu^{2}}{8}-\frac{979 \nu}{12}\right) X_{1}\right]+\frac{1}{2}\left(-\frac{47 \nu^{3}}{4}-\frac{4771 \nu^{2}}{24}-42 \nu\right) \boldsymbol{n} \cdot \chi_{1} \boldsymbol{n} \cdot \chi_{2}\right\}+1 \leftrightarrow 2, \\
& B_{n p}^{S S}=\frac{1}{\hat{r}^{3}}\left\{\chi_{1}^{2}\left[\left(-3 \nu^{2}+3 \nu+(3 \nu-3) X_{1}\right) \tilde{C}_{1\left(\mathrm{ES}^{2}\right)}-\frac{15 \nu^{2}}{4}+9 \nu X_{1}\right]+\frac{1}{2} \chi_{1} \cdot \chi_{2}\left(\frac{9 \nu^{2}}{2}+6 \nu\right)\right\} \\
& +\frac{1}{\hat{r}^{4}}\left\{\chi_{1}^{2}\left[\left(-\frac{159 \nu^{2}}{4}+\frac{23 \nu}{2}+\left(-12 \nu^{2}+\frac{197 \nu}{4}-\frac{23}{2}\right) X_{1}\right) \tilde{C}_{1\left(\mathrm{ES}^{2}\right)}+5 \nu^{3}-55 \nu^{2}+\left(\frac{251 \nu}{4}-37 \nu^{2}\right) X_{1}\right]\right. \\
& \left.+\frac{1}{2} \chi_{1} \cdot \chi_{2}\left(10 \nu^{3}+26 \nu^{2}+20 \nu\right)\right\}+1 \leftrightarrow 2 \\
& Q^{S^{2}}=\frac{\hat{p}_{r}^{3}}{\hat{r}^{3}}\left\{\boldsymbol{n} \cdot \chi_{1} \hat{\boldsymbol{p}} \cdot \chi_{1}\left[\left(20 \nu^{3}-35 \nu^{2}+\left(35 \nu-20 \nu^{2}\right) X_{1}\right) \tilde{\boldsymbol{C}}_{1\left(\mathrm{ES}^{2}\right)}+\frac{199 \nu^{3}}{8}-\frac{1085 \nu^{2}}{24}+\left(\frac{130 \nu}{3}-\frac{517 \nu^{2}}{8}\right) X_{1}\right]\right. \\
& \left.+\boldsymbol{n} \cdot \chi_{1} \hat{\boldsymbol{p}} \cdot \chi_{2}\left(-\frac{79 \nu^{3}}{8}+\frac{79 \nu^{2}}{12}+\frac{45 \nu}{16}\right)\right\} \\
& +\frac{\hat{p}_{r}^{4}}{\hat{r}^{3}}\left\{\chi_{1}^{2}\left[\left(5 \nu^{3}-\frac{35 \nu^{2}}{4}+\left(\frac{35 \nu}{4}-5 \nu^{2}\right) X_{1}\right) \tilde{C}_{1\left(\mathrm{ES}^{2}\right)}+\frac{55 \nu^{3}}{8}-\frac{105 \nu^{2}}{8}+\left(\frac{55 \nu}{4}-\frac{145 \nu^{2}}{8}\right) X_{1}\right]\right. \\
& +\left(\boldsymbol{n} \cdot \chi_{1}\right)^{2}\left[\left(\frac{245 \nu^{2}}{4}-35 \nu^{3}+\left(35 \nu^{2}-\frac{245 \nu}{4}\right) X_{1}\right) \tilde{C}_{1\left(\mathrm{ES}^{2}\right)}-\frac{91 \nu^{3}}{2}+\frac{1015 \nu^{2}}{12}+\left(119 \nu^{2}-\frac{1015 \nu}{12}\right) X_{1}\right] \\
& \left.+\frac{1}{2} \chi_{1} \cdot \chi_{2}\left(-\frac{25 \nu^{3}}{4}+\frac{45 \nu^{2}}{8}+\frac{5 \nu}{2}\right)+\frac{1}{2} \boldsymbol{n} \cdot \chi_{1} \boldsymbol{n} \cdot \chi_{2}\left(\frac{77 \nu^{3}}{2}-\frac{721 \nu^{2}}{24}-\frac{105 \nu}{8}\right)\right\}+1 \leftrightarrow 2,
\end{aligned}
$$

$$
\begin{aligned}
A^{S^{4}}= & \frac{1}{\hat{r}^{5}}\left\{\left(\boldsymbol{n} \cdot \chi_{1}\right)^{4}\left[\left(\frac{15 \nu^{2}}{2}-\frac{15 \nu}{2}+\left(\frac{15}{2}-15 \nu\right) X_{1}\right) \tilde{C}_{1\left(\mathrm{ES}^{2}\right)}+\left(\frac{35 \nu}{4}-\frac{35 \nu^{2}}{4}+\left(\frac{35 \nu}{2}-\frac{35}{4}\right) X_{1}\right) \tilde{C}_{1\left(\mathrm{ES}^{4}\right)}\right]\right. \\
& +\boldsymbol{n} \cdot \chi_{2}\left(\boldsymbol{n} \cdot \chi_{1}\right)^{3}\left[\left(35 \nu^{2}-35 \nu X_{1}\right) \tilde{C}_{1\left(\mathrm{BS}^{3}\right)}+\left(15 \nu X_{1}-15 \nu^{2}\right) \tilde{C}_{1\left(\mathrm{ES}^{2}\right)}\right] \\
& +\chi_{1} \cdot \chi_{2}\left(\boldsymbol{n} \cdot \chi_{1}\right)^{2}\left[\left(15 \nu X_{1}-15 \nu^{2}\right) \tilde{C}_{1\left(\mathrm{BS}^{3}\right)}+\left(12 \nu^{2}-12 \nu X_{1}\right) \tilde{C}_{1\left(\mathrm{ES}^{2}\right)}\right] \\
& +\chi_{1}^{2}\left(\boldsymbol{n} \cdot \chi_{1}\right)^{2}\left[\left(-9 \nu^{2}+9 \nu+(18 \nu-9) X_{1}\right) \tilde{C}_{1\left(\mathrm{ES}^{2}\right)}+\left(\frac{15 \nu^{2}}{2}-\frac{15 \nu}{2}+\left(\frac{15}{2}-15 \nu\right) X_{1}\right) \tilde{C}_{\left.1\left(\mathrm{ES}^{4}\right)\right]}\right] \\
& +\chi_{2}^{2}\left(\boldsymbol{n} \cdot \chi_{1}\right)^{2}\left[6 \tilde{C}_{2\left(\mathrm{ES}^{2}\right)} \nu^{2}+\tilde{C}_{1\left(\mathrm{ES}^{2}\right)}\left(\frac{15}{2} \tilde{\boldsymbol{C}}_{2\left(\mathrm{ES}^{2}\right)^{2}}+3 \nu^{2}\right)\right] \\
& +\frac{1}{2}\left(\boldsymbol{n} \cdot \chi_{2}\right)^{2}\left(\boldsymbol{n} \cdot \chi_{1}\right)^{2}\left[\tilde{C}_{1\left(\mathrm{ES}^{2}\right)}\left(-\frac{1}{2} 105 \tilde{C}_{2\left(\mathrm{ES}^{2}\right)} \nu^{2}-45 \nu^{2}\right)-45 \nu^{2} \tilde{C}_{2\left(\mathrm{ES}^{2}\right)}\right]
\end{aligned}
$$




$$
\begin{aligned}
& +\boldsymbol{n} \cdot \chi_{2} \chi_{1}^{2} \boldsymbol{n} \cdot \chi_{1}\left[\left(15 \nu X_{1}-15 \nu^{2}\right) \tilde{C}_{1\left(\mathrm{BS}^{3}\right)}+\left(6 \nu^{2}-6 \nu X_{1}\right) \tilde{\boldsymbol{C}}_{1\left(\mathrm{ES}^{2}\right)}\right] \\
& +\frac{1}{2} \boldsymbol{n} \cdot \chi_{2} \chi_{1} \cdot \chi_{2} \boldsymbol{n} \cdot \chi_{1}\left[27 \tilde{C}_{2\left(\mathrm{ES}^{2}\right)} \nu^{2}+\tilde{C}_{1\left(\mathrm{ES}^{2}\right)}\left(30 \tilde{C}_{2\left(\mathrm{ES}^{2}\right)} \nu^{2}+27 \nu^{2}\right)\right] \\
& +\chi_{1} \cdot \chi_{2} \chi_{1}^{2}\left[\left(3 \nu^{2}-3 \nu X_{1}\right) \tilde{C}_{1\left(\mathrm{BS}^{3}\right)}+\left(3 \nu X_{1}-3 \nu^{2}\right) \tilde{C}_{1\left(\mathrm{ES}^{2}\right)}\right]-\frac{3}{4} \nu^{2} \chi_{1}^{2} \chi_{2}^{2} \tilde{\boldsymbol{C}}_{1\left(\mathrm{ES}^{2}\right)} \tilde{C}_{2\left(\mathrm{ES}^{2}\right)} \\
& +\chi_{1}^{4}\left[\left(\frac{3 \nu^{2}}{2}-\frac{3 \nu}{2}+\left(\frac{3}{2}-3 \nu\right) X_{1}\right) \tilde{C}_{1\left(\mathrm{ES}^{2}\right)}+\left(-\frac{3 \nu^{2}}{4}+\frac{3 \nu}{4}+\left(\frac{3 \nu}{2}-\frac{3}{4}\right) X_{1}\right) \tilde{C}_{1\left(\mathrm{ES}^{4}\right)}\right] \\
& \left.+\frac{1}{2}\left(\chi_{1} \cdot \chi_{2}\right)^{2}\left[\tilde{C}_{1\left(\mathrm{ES}^{2}\right)}\left(-3 \tilde{C}_{2\left(\mathrm{ES}^{2}\right)} \nu^{2}-3 \nu^{2}\right)-3 \nu^{2} \tilde{C}_{2\left(\mathrm{ES}^{2}\right)}\right]\right\}+1 \leftrightarrow 2 .
\end{aligned}
$$

\section{Coefficients of the $\mathrm{SEOB}_{\mathrm{TM}}^{r_{c} \text {,align }}$ Hamiltonian}

The coefficients of the $\mathrm{SEOB}_{\mathrm{TM}}^{r_{c} \text {,align }}$ Hamiltonian are given in Ref. [47], but we rewrite them here for convenience in the notation used in the rest of the paper.

The spin-orbit and spin-cubic correction in Eq. (39) are given by

$$
\begin{aligned}
G_{S}= & 2\left[1+\frac{27}{16} \nu \hat{p}_{r_{*}}^{2}+\frac{5 \nu}{16 \hat{r}_{c}}+\left(\frac{169 \nu^{2}}{256}-\frac{5 \nu}{16}\right) \hat{p}_{r_{*}}^{4}+\frac{\hat{p}_{r_{*}}^{2}}{\hat{r}_{c}}\left(12 \nu-\frac{49 \nu^{2}}{128}\right)+\frac{1}{\hat{r}_{c}^{2}}\left(\frac{41 \nu^{2}}{256}+\frac{51 \nu}{8}\right)\right]^{-1}, \\
G_{S^{*}}= & \frac{3}{2}\left[1+\frac{1}{\hat{r}_{c}}\left(\frac{\nu}{2}+\frac{3}{4}\right)+\left(\frac{3 \nu}{2}+\frac{5}{4}\right) \hat{p}_{r_{*}}^{2}+\left(\frac{3 \nu^{2}}{8}+\frac{25 \nu}{12}+\frac{5}{48}\right) \hat{p}_{r_{*}}^{4}+\frac{\hat{p}_{r_{*}}^{2}}{\hat{r}_{c}}\left(-\frac{7 \nu^{2}}{8}+11 \nu+4\right)\right. \\
& \left.+\frac{1}{\hat{r}_{c}^{2}}\left(\frac{3 \nu^{2}}{8}+\frac{29 \nu}{4}+\frac{27}{16}\right)\right]^{-1}, \\
G_{S^{3}}= & \frac{1}{\hat{r}_{c}}\left\{\chi _ { 1 } ^ { 2 } \left[\left(1-2 \nu+(\nu-1) X_{1}\right) \tilde{C}_{1\left(\mathrm{BS}^{3}\right)}+\left(\frac{\nu^{2}}{4}+\frac{5 \nu}{4}+\left(\frac{3}{4}-\frac{\nu}{2}\right) X_{1}-\frac{3}{4}\right) \tilde{C}_{1\left(\mathrm{ES}^{2}\right)}+\frac{\nu^{2}}{4}-\frac{3 \nu}{4}+\frac{1}{4}\right.\right. \\
& \left.\left.+\left(\frac{\nu}{2}-\frac{1}{4}\right) X_{1}\right]+\chi_{1} \chi_{2}\left[\left(\frac{\nu^{2}}{4}+2 \nu-2 \nu X_{1}\right) \tilde{C}_{1\left(\mathrm{ES}^{2}\right)}-\frac{\nu^{2}}{4}+\frac{\nu}{2}-\frac{\nu X_{1}}{2}\right]\right\}, \\
\tilde{G}_{S^{3}}= & G_{S^{3}} \quad \text { with } 1 \leftrightarrow 2 .
\end{aligned}
$$

The spin-squared and spin-quartic corrections in Eq. (37) are given by

$$
\begin{aligned}
& \delta a_{\mathrm{NLO}}^{2}=\chi_{1}^{2}\left[\left((4-2 \nu) X_{1}-4 \nu\right) \tilde{C}_{1\left(\mathrm{ES}^{2}\right)}+\frac{\nu^{2}}{2}-3 \nu X_{1}\right]+\frac{1}{2} \chi_{1} \chi_{2}\left(\nu^{2}-2 \nu\right)+1 \leftrightarrow 2, \\
& \delta a_{\mathrm{NNLO}}^{2}=\chi_{1}^{2}\left[\left(\frac{207 \nu^{2}}{28}-\frac{275 \nu}{14}+\left(\frac{275}{14}-\frac{533 \nu}{28}\right) X_{1}\right) \tilde{C}_{1\left(\mathrm{ES}^{2}\right)}-\frac{3 \nu^{3}}{8}+\frac{157 \nu^{2}}{8}+\left(\frac{45 \nu^{2}}{8}-\frac{123 \nu}{4}\right) X_{1}\right] \\
& +\frac{1}{2} \chi_{1} \chi_{2}\left(-\frac{3 \nu^{3}}{4}-\frac{145 \nu^{2}}{8}-\frac{25 \nu}{2}\right)+1 \leftrightarrow 2 \text {, } \\
& \delta a_{\mathrm{LO}}^{4}=\chi_{1}^{3} \chi_{2}\left[\left(3 \nu X_{1}-3 \nu^{2}\right) \tilde{C}_{1\left(\mathrm{BS}^{3}\right)}+\left(3 \nu^{2}-3 \nu X_{1}\right) \tilde{C}_{1\left(\mathrm{ES}^{2}\right)}\right]+\frac{1}{2} \chi_{1}^{2} \chi_{2}^{2}\left[\tilde{C}_{1\left(\mathrm{ES}^{2}\right)}\left(3 \nu^{2} \tilde{C}_{2\left(\mathrm{ES}^{2}\right)}+3 \nu^{2}\right)+3 \nu^{2} \tilde{C}_{2\left(\mathrm{ES}^{2}\right)}\right] \\
& +\chi_{1}^{4}\left[\left(-\frac{3 \nu^{2}}{4}+\frac{9 \nu}{4}+\left(\frac{3}{4}-\frac{3 \nu}{2}\right) X_{2}-\frac{3}{4}\right) \tilde{C}_{1\left(\mathrm{ES}^{2}\right)}^{2}+\left(-\frac{3 \nu^{2}}{2}+\frac{9 \nu}{2}+\left(\frac{3}{2}-3 \nu\right) X_{2}-\frac{3}{2}\right) \tilde{C}_{1\left(\mathrm{ES}^{2}\right)}\right. \\
& \left.+\left(\frac{3 \nu^{2}}{4}-\frac{9 \nu}{4}+\left(\frac{3 \nu}{2}-\frac{3}{4}\right) X_{2}+\frac{3}{4}\right) \tilde{C}_{1\left(\mathrm{ES}^{4}\right)}\right]+1 \leftrightarrow 2 .
\end{aligned}
$$




\section{Coefficients of the $\mathrm{SEOB}_{\mathrm{TS}}$ Hamiltonian}

The spin-squared corrections in Eq. (40) are given by

$$
\begin{aligned}
A^{S S}= & \frac{1}{\hat{r}^{3}} \chi_{1}^{2}\left(\nu-X_{1}\right) \tilde{C}_{1\left(\mathrm{ES}^{2}\right)} \\
& +\frac{1}{\hat{r}^{4}}\left\{\chi_{1}^{2}\left[\left(2 \nu+(2 \nu-2) X_{1}\right) \tilde{C}_{1\left(\mathrm{ES}^{2}\right)}+\frac{17 \nu^{2}}{6}+\left(\frac{23 \nu^{2}}{6}+\frac{\nu}{6}\right) X_{1}\right]+\frac{37}{12} \nu^{2} \chi_{1} \cdot \chi_{2}\right\} \\
& +\frac{1}{\hat{r}^{5}}\left\{\chi _ { 1 } ^ { 2 } \left[\left(-\frac{207 \nu^{2}}{28}+\frac{51 \nu}{14}+\left(\frac{309 \nu}{28}-\frac{51}{14}\right) X_{1}\right) \tilde{C}_{1\left(\mathrm{ES}^{2}\right)}+\frac{529 \nu^{4}}{144}-\frac{2353 \nu^{3}}{144}+\frac{55 \nu^{2}}{4}\right.\right. \\
& \left.\left.+\left(\frac{143 \nu^{3}}{72}+\frac{7015 \nu^{2}}{144}-\frac{155 \nu}{8}\right) X_{1}\right]+\frac{1}{2} \chi_{1} \cdot \chi_{2}\left(\frac{529 \nu^{4}}{72}-\frac{112 \nu^{3}}{3}+\frac{51 \nu^{2}}{8}\right)\right\}+1 \leftrightarrow 2,
\end{aligned}
$$

$$
\begin{aligned}
A^{n S}= & \frac{1}{\hat{r}^{3}}\left(3 X_{1}-3 \nu\right) \tilde{C}_{1\left(\mathrm{ES}^{2}\right)}\left(\boldsymbol{n} \cdot \chi_{1}\right)^{2} \\
& +\frac{1}{\hat{r}^{4}}\left\{\left(\boldsymbol{n} \cdot \chi_{1}\right)^{2}\left[\left(-3 \nu^{2}-3 \nu+(3-3 \nu) X_{1}\right) \tilde{C}_{1\left(\mathrm{ES}^{2}\right)}-\frac{5 \nu^{3}}{4}+\frac{3 \nu^{2}}{2}+\left(-\frac{15 \nu^{2}}{4}-6 \nu\right) X_{1}\right]\right. \\
& \left.+\frac{1}{2} \boldsymbol{n} \cdot \chi_{1} \boldsymbol{n} \cdot \chi_{2}\left(-\frac{5 \nu^{3}}{2}-\frac{67 \nu^{2}}{4}\right)\right\} \\
& +\frac{1}{\hat{r}^{5}}\left\{( \boldsymbol { n } \cdot \chi _ { 1 } ) ^ { 2 } \left[\left(\frac{\nu^{3}}{4}-\frac{205 \nu^{2}}{28}-\frac{24 \nu}{7}+\left(-\frac{37 \nu^{2}}{4}+\frac{64 \nu}{7}+\frac{24}{7}\right) X_{1}\right) \tilde{\boldsymbol{C}}_{1\left(\mathrm{ES}^{2}\right)}-\frac{109 \nu^{4}}{12}+\frac{113 \nu^{3}}{4}-\frac{515 \nu^{2}}{12}\right.\right. \\
& \left.\left.+\left(-\frac{13 \nu^{3}}{2}-\frac{374 \nu^{2}}{3}+\frac{149 \nu}{3}\right) X_{1}\right]+\frac{1}{2} \boldsymbol{n} \cdot \chi_{1} \boldsymbol{n} \cdot \chi_{2}\left(-\frac{109 \nu^{4}}{6}+\frac{133 \nu^{3}}{12}-\frac{1301 \nu^{2}}{48}\right)\right\}+1 \leftrightarrow 2
\end{aligned}
$$

$$
\begin{aligned}
B_{p}^{n S}= & \frac{1}{\hat{r}^{3}}\left\{\left(\boldsymbol{n} \cdot \chi_{1}\right)^{2}\left[\left(3 \nu^{2}-3 \nu+(3-3 \nu) X_{1}\right) \tilde{C}_{1\left(\mathrm{ES}^{2}\right)}+\frac{5 \nu^{3}}{4}-\frac{29 \nu^{2}}{4}+\left(\frac{13 \nu}{2}-\frac{31 \nu^{2}}{4}\right) X_{1}\right]+\frac{1}{2} \boldsymbol{n} \cdot \chi_{1} \boldsymbol{n} \cdot \chi_{2}\left(\frac{5 \nu^{3}}{2}+\frac{15 \nu^{2}}{4}\right)\right\} \\
& +\frac{1}{\hat{r}^{4}}\left\{( \boldsymbol { n } \cdot \chi _ { 1 } ) ^ { 2 } \left[\left(-\frac{\nu^{3}}{4}+\frac{59 \nu^{2}}{2}-\frac{15 \nu}{2}+\left(\frac{37 \nu^{2}}{4}-\frac{169 \nu}{4}+\frac{15}{2}\right) X_{1}\right) \tilde{C}_{1\left(\mathrm{ES}^{2}\right)}-\frac{31 \nu^{4}}{16}-\frac{413 \nu^{3}}{48}-\frac{101 \nu^{2}}{24}\right.\right. \\
& \left.\left.+\left(\frac{13 \nu^{3}}{24}+\frac{763 \nu^{2}}{48}+\frac{155 \nu}{24}\right) X_{1}\right]+\frac{1}{2} \boldsymbol{n} \cdot \chi_{1} \boldsymbol{n} \cdot \chi_{2}\left(-\frac{31 \nu^{4}}{8}+\frac{217 \nu^{3}}{6}+\frac{1493 \nu^{2}}{48}\right)\right\}+1 \leftrightarrow 2,
\end{aligned}
$$

$$
\begin{aligned}
B_{n p}^{n S}= & \frac{1}{\hat{r}^{3}}\left\{\left(\boldsymbol{n} \cdot \chi_{1}\right)^{2}\left(-\frac{15 \nu^{3}}{4}+15 \nu^{2}+\left(\frac{75 \nu^{2}}{4}-15 \nu\right) X_{1}\right)+\frac{1}{2} \boldsymbol{n} \cdot \chi_{1} \boldsymbol{n} \cdot \chi_{2}\left(-\frac{15 \nu^{3}}{2}-\frac{45 \nu^{2}}{4}\right)\right\} \\
& +\frac{1}{\hat{r}^{4}}\left\{( \boldsymbol { n } \cdot \chi _ { 1 } ) ^ { 2 } \left[\left(\nu^{3}-\frac{31 \nu^{2}}{4}+\frac{9 \nu}{2}+\left(-\nu^{2}+\frac{121 \nu}{4}-\frac{9}{2}\right) X_{1}\right) \tilde{C}_{1\left(\mathrm{ES}^{2}\right)}-\frac{63 \nu^{4}}{16}+\frac{2615 \nu^{3}}{48}-\frac{899 \nu^{2}}{24}\right.\right. \\
& \left.\left.+\left(\frac{509 \nu^{3}}{24}-\frac{1331 \nu^{2}}{16}+\frac{1223 \nu}{24}\right) X_{1}\right]+\frac{1}{2} \boldsymbol{n} \cdot \chi_{1} \boldsymbol{n} \cdot \chi_{2}\left(-\frac{63 \nu^{4}}{8}-\frac{455 \nu^{3}}{12}+\frac{63 \nu^{2}}{4}\right)\right\}+1 \leftrightarrow 2,
\end{aligned}
$$

$$
\begin{aligned}
B_{n p}^{S S}= & \frac{1}{\hat{r}^{3}} \chi_{1}^{2}\left[\left(-3 \nu^{2}+3 \nu+(3 \nu-3) X_{1}\right) \tilde{C}_{1\left(\mathrm{ES}^{2}\right)}+\frac{9 \nu^{2}}{4}+\left(\frac{3 \nu^{2}}{2}-\frac{3 \nu}{2}\right) X_{1}\right] \\
& +\frac{1}{\hat{r}^{4}}\left\{\chi _ { 1 } ^ { 2 } \left[\left(-\frac{135 \nu^{2}}{4}+\frac{11 \nu}{2}+\left(-12 \nu^{2}+\frac{173 \nu}{4}-\frac{11}{2}\right) X_{1}\right) \tilde{C}_{1\left(\mathrm{ES}^{2}\right)}+\frac{187 \nu^{4}}{48}-\frac{409 \nu^{3}}{48}+\frac{781 \nu^{2}}{72}\right.\right. \\
& \left.\left.+\left(-\frac{187 \nu^{3}}{24}+\frac{605 \nu^{2}}{144}-\frac{1807 \nu}{72}\right) X_{1}\right]+\frac{1}{2} \chi_{1} \cdot \chi_{2}\left(\frac{187 \nu^{4}}{24}-\frac{181 \nu^{3}}{4}-\frac{1213 \nu^{2}}{18}\right)\right\}+1 \leftrightarrow 2
\end{aligned}
$$




$$
\begin{aligned}
Q^{S^{2}}= & \frac{\hat{p}_{r}^{3}}{\hat{r}^{3}}\left\{\boldsymbol { n } \cdot \chi _ { 1 } \hat { \boldsymbol { p } } \cdot \chi _ { 1 } \left[\left(20 \nu^{3}-35 \nu^{2}+\left(35 \nu-20 \nu^{2}\right) X_{1}\right) \tilde{C}_{1\left(\mathrm{ES}^{2}\right)}+\frac{45 \nu^{4}}{8}-\frac{425 \nu^{3}}{12}+\frac{35 \nu^{2}}{6}\right.\right. \\
& \left.\left.+\left(-\frac{100 \nu^{3}}{3}+\frac{145 \nu^{2}}{4}-\frac{35 \nu}{6}\right) X_{1}\right]+\boldsymbol{n} \cdot \chi_{1} \hat{\boldsymbol{p}} \cdot \chi_{2}\left[\frac{45 \nu^{4}}{8}+\frac{10 \nu^{3}}{3}-\frac{25 \nu^{2}}{16}\right]\right\} \\
& +\frac{\hat{p}_{r}^{4}}{\hat{r}^{3}}\left\{( \boldsymbol { n } \cdot \chi _ { 1 } ) ^ { 2 } \left[\left(-35 \nu^{3}+\frac{245 \nu^{2}}{4}+\left(35 \nu^{2}-\frac{245 \nu}{4}\right) X_{1}\right) \tilde{C}_{1\left(\mathrm{ES}^{2}\right)}-\frac{105 \nu^{4}}{16}+\frac{2135 \nu^{3}}{48}-\frac{35 \nu^{2}}{24}\right.\right. \\
& \left.+\left(\frac{1085 \nu^{3}}{24}-\frac{595 \nu^{2}}{16}+\frac{35 \nu}{24}\right) X_{1}\right]+\frac{1}{2} \boldsymbol{n} \cdot \chi_{1} \boldsymbol{n} \cdot \chi_{2}\left[-\frac{105 \nu^{4}}{8}-\frac{245 \nu^{3}}{12}+\frac{35 \nu^{2}}{8}\right] \\
& +\chi_{1}^{2}\left[\left(5 \nu^{3}-\frac{35 \nu^{2}}{4}+\left(\frac{35 \nu}{4}-5 \nu^{2}\right) X_{1}\right) \tilde{C}_{1\left(\mathrm{ES}^{2}\right)}+\frac{5 \nu^{4}}{16}-\frac{145 \nu^{3}}{48}-\frac{35 \nu^{2}}{24}\right. \\
& \left.\left.+\left(-\frac{95 \nu^{3}}{24}+\frac{5 \nu^{2}}{16}+\frac{35 \nu}{24}\right) X_{1}\right]+\frac{1}{2} \chi_{1} \cdot \chi_{2}\left[\frac{5 \nu^{4}}{8}+\frac{55 \nu^{3}}{12}-\frac{5 \nu^{2}}{12}\right]\right\}+1 \leftrightarrow 2 .
\end{aligned}
$$

The spin-cubed corrections in Eq. (49b) are

$$
\begin{aligned}
G_{S^{3}}= & \frac{1}{\hat{r}}\left\{( \boldsymbol { n } \cdot \chi _ { 1 } ) ^ { 2 } \left[\left(5 \nu+(5 \nu-5) X_{1}\right) \tilde{C}_{1\left(\mathrm{BS}^{3}\right)}+\left(-\frac{3 \nu^{3}}{2}-\frac{9 \nu^{2}}{4}+\left(3 \nu^{2}+\frac{9 \nu}{4}\right) X_{1}\right) \tilde{C}_{1\left(\mathrm{ES}^{2}\right)}-\nu^{4}+7 \nu^{3}+\frac{5 \nu^{2}}{4}\right.\right. \\
& \left.+\left(3 \nu^{3}-7 \nu^{2}-\frac{5 \nu}{4}\right) X_{1}\right]+\chi_{1}^{2}\left[\left((1-\nu) X_{1}-\nu\right) \tilde{C}_{1\left(\mathrm{BS}^{3}\right)}+\left(\frac{3 \nu^{2}}{4}-\frac{3 \nu X_{1}}{4}\right) \tilde{C}_{1\left(\mathrm{ES}^{2}\right)}-\frac{5 \nu^{3}}{4}+\frac{\left.3 \nu^{2} X_{1}\right]}{2}\right] \\
& +\boldsymbol{n} \cdot \chi_{1} \boldsymbol{n} \cdot \chi_{2}\left[\left(-\frac{7 \nu^{3}}{2}-\frac{5 \nu^{2}}{2}-\frac{15 \nu X_{1}}{2}\right) \tilde{C}_{1\left(\mathrm{ES}^{2}\right)}-2 \nu^{4}+\frac{37 \nu^{3}}{6}-5 \nu^{2}+\left(\frac{\nu^{3}}{3}-\frac{11 \nu^{2}}{3}\right) X_{1}\right] \\
& +\chi_{1} \cdot \chi_{2}\left[\left(\nu^{3}+\frac{\nu^{2}}{2}+\frac{3 \nu X_{1}}{2}\right) \tilde{C}_{1\left(\mathrm{ES}^{2}\right)}+\frac{17 \nu^{3}}{12}+\nu^{2}+\left(\frac{5 \nu^{3}}{6}-\frac{7 \nu^{2}}{6}\right) X_{1}\right] \\
& +\left(\boldsymbol{n} \cdot \chi_{2}\right)^{2}\left[\left(2 \nu^{3}-\frac{5 \nu^{2}}{4}-\frac{15 \nu X_{2}}{4}\right) \tilde{C}_{2\left(\mathrm{ES}^{2}\right)}-\nu^{4}+\frac{89 \nu^{3}}{6}-\frac{5 \nu^{2}}{2}+\left(\frac{8 \nu^{3}}{3}-\frac{25 \nu^{2}}{3}\right) X_{2}\right] \\
& \left.+\chi_{2}^{2}\left[\left(-\nu^{3}+\frac{\nu^{2}}{4}+\frac{3 \nu X_{2}}{4}\right) \tilde{C}_{2\left(\mathrm{ES}^{2}\right)}-\frac{31 \nu^{3}}{6}+\frac{\nu^{2}}{2}+\left(\frac{8 \nu^{2}}{3}-\frac{5 \nu^{3}}{6}\right) X_{2}\right]\right\} \\
& +\hat{L}^{2}\left[\left(\boldsymbol{n} \cdot \chi_{1}\right)^{2}\left(\frac{\nu^{4}}{2}-\frac{3 \nu^{3}}{2}+\frac{\nu^{2}}{2}+\left(-\frac{3 \nu^{3}}{2}+2 \nu^{2}-\frac{\nu}{2}\right) X_{1}\right)+\boldsymbol{n} \cdot \chi_{1} \boldsymbol{n} \cdot \chi_{2}\left(\nu^{4}-\nu^{3} X_{1}\right)+\left(\boldsymbol{n} \cdot \chi_{2}\right)^{2}\left(\frac{\nu^{4}}{2}-\frac{\nu^{3} X_{2}}{2}\right)\right] \\
& +\hat{p}_{r}^{2}\left[\left(\boldsymbol{n} \cdot \chi_{1}\right)^{2}\left(-\frac{5 \nu^{4}}{2}+\frac{15 \nu^{3}}{2}-\frac{5 \nu^{2}}{2}+\left(\frac{15 \nu^{3}}{2}-10 \nu^{2}+\frac{5 \nu}{2}\right) X_{1}\right)+\boldsymbol{n} \cdot \chi_{1} \boldsymbol{n} \cdot \chi_{2}\left(5 \nu^{3} X_{1}-5 \nu^{4}\right)\right. \\
& \left.+\left(\boldsymbol{n} \cdot \chi_{2}\right)^{2}\left(\frac{5 \nu^{3} X_{2}}{2}-\frac{5 \nu^{4}}{2}\right)\right],
\end{aligned}
$$

$\tilde{G}_{S^{3}}=G_{S^{3}} \quad$ with $\quad 1 \leftrightarrow 2$. 
The spin-quartic corrections in Eq. (49) read

$$
\begin{aligned}
& A^{S^{4}}=\frac{1}{\hat{r}^{5}}\left\{( \boldsymbol { n } \cdot \chi _ { 1 } ) ^ { 4 } \left[-\frac{63 \nu^{4}}{4}+36 \nu^{3}+3 \nu^{2}+\left(-\frac{15 \nu^{3}}{2}+\frac{45 \nu^{2}}{2}-\frac{15 \nu}{2}+\left(\frac{45 \nu^{2}}{2}-30 \nu+\frac{15}{2}\right) X_{1}\right) \tilde{\boldsymbol{C}}_{1\left(\mathrm{ES}^{2}\right)}\right.\right. \\
& \left.+\left(46 \nu^{3}-33 \nu^{2}-3 \nu\right) X_{1}+\left(-\frac{35 \nu^{2}}{4}+\frac{35 \nu}{4}+\left(\frac{35 \nu}{2}-\frac{35}{4}\right) X_{1}\right) \tilde{C}_{1\left(\mathrm{ES}^{4}\right)}\right] \\
& +\boldsymbol{n} \cdot \chi_{2}\left(\boldsymbol{n} \cdot \chi_{1}\right)^{3}\left[-3 \nu^{4}+3 \nu^{3}+\left(6 \nu^{3}-6 \nu^{2}\right) X_{1}+\left(3 \nu^{2}-3 \nu X_{1}\right) \tilde{\boldsymbol{C}}_{1\left(\mathrm{BS}^{3}\right)}+\left(3 \nu^{2} X_{1}-3 \nu^{3}\right) \tilde{\boldsymbol{C}}_{1\left(\mathrm{ES}^{2}\right)}\right] \\
& +\chi_{1}^{2}\left(\boldsymbol{n} \cdot \chi_{1}\right)^{2}\left[\frac{21 \nu^{4}}{2}-27 \nu^{3}-3 \nu^{2}+\left(9 \nu^{3}-27 \nu^{2}+9 \nu+\left(-27 \nu^{2}+36 \nu-9\right) X_{1}\right) \tilde{C}_{1\left(\mathrm{ES}^{2}\right)}\right. \\
& \left.+\left(-33 \nu^{3}+24 \nu^{2}+3 \nu\right) X_{1}+\left(\frac{15 \nu^{2}}{2}-\frac{15 \nu}{2}+\left(\frac{15}{2}-15 \nu\right) X_{1}\right) \tilde{C}_{1\left(\mathrm{ES}^{4}\right)}\right] \\
& +\chi_{2}^{2}\left(\boldsymbol{n} \cdot \chi_{1}\right)^{2}\left[-63 \nu^{4}+58 \nu^{3}+\left(35 \nu^{2}-35 \nu X_{2}\right) \tilde{C}_{2\left(\mathrm{BS}^{3}\right)}\right. \\
& \left.+\left(104 \nu^{3}-93 \nu^{2}\right) X_{2}+\left(\left(6 \nu^{3}+12 \nu^{2}\right) X_{2}-12 \nu^{3}\right) \tilde{C}_{2\left(\mathrm{ES}^{2}\right)}\right] \\
& +\chi_{1} \cdot \chi_{2}\left(\boldsymbol{n} \cdot \chi_{1}\right)^{2}\left[-\frac{189 \nu^{4}}{2}+54 \nu^{3}-42 \nu^{2}+\left(-\frac{21 \nu^{3}}{2}-\frac{105 \nu^{2}}{2}+\left(\frac{21 \nu^{2}}{2}-6 \nu^{3}\right) X_{1}\right) \tilde{C}_{2\left(\mathrm{ES}^{2}\right)}\right. \\
& \left.+\tilde{C}_{1\left(\mathrm{ES}^{2}\right)}\left(-\frac{33 \nu^{3}}{2}-\frac{105}{2} \tilde{C}_{2\left(\mathrm{ES}^{2}\right)} \nu^{2}-42 \nu^{2}+\left(6 \nu^{3}-\frac{21 \nu^{2}}{2}\right) X_{1}\right)\right] \\
& +\frac{1}{2}\left(\boldsymbol{n} \cdot \chi_{2}\right)^{2}\left(\boldsymbol{n} \cdot \chi_{1}\right)^{2}\left[\frac{21 \nu^{4}}{2}-27 \nu^{3}-3 \nu^{2}+\left(9 \nu^{3}-27 \nu^{2}+9 \nu+\left(-27 \nu^{2}+36 \nu-9\right) X_{2}\right) \tilde{C}_{2\left(\mathrm{ES}^{2}\right)}\right. \\
& \left.+\left(-33 \nu^{3}+24 \nu^{2}+3 \nu\right) X_{2}+\left(\frac{15 \nu^{2}}{2}-\frac{15 \nu}{2}+\left(\frac{15}{2}-15 \nu\right) X_{2}\right) \tilde{C}_{2\left(\mathrm{ES}^{4}\right)}\right] \\
& +\boldsymbol{n} \cdot \chi_{2} \chi_{1}^{2} \boldsymbol{n} \cdot \chi_{1}\left[\frac{23 \nu^{4}}{2}-\frac{15 \nu^{3}}{2}+\frac{9 \nu^{2}}{2}+\left(-2 \nu^{4}-6 \nu^{3}+\frac{9 \nu^{2}}{2}\right) X_{1}+\left(\frac{3 \nu^{3}}{2}-\frac{3 X_{1} \nu^{2}}{2}+\frac{15 \nu^{2}}{2}\right) \tilde{C}_{2\left(\mathrm{ES}^{2}\right)}\right. \\
& \left.+\tilde{C}_{1\left(\mathrm{ES}^{2}\right)}\left(\frac{9 \nu^{3}}{2}+\frac{9 X_{1} \nu^{2}}{2}+\frac{15}{2} \tilde{C}_{2\left(\mathrm{ES}^{2}\right)} \nu^{2}+3 \nu^{2}\right)\right] \\
& +\frac{1}{2} \boldsymbol{n} \cdot \chi_{2} \chi_{1} \cdot \chi_{2} \boldsymbol{n} \cdot \chi_{1}\left[\frac{23 \nu^{4}}{2}-\frac{15 \nu^{3}}{2}+\frac{9 \nu^{2}}{2}+\left(\frac{9 \nu^{2}}{2}-2 \nu^{4}-6 \nu^{3}\right) X_{2}+\left(\frac{9 \nu^{3}}{2}+\frac{9 X_{2} \nu^{2}}{2}+3 \nu^{2}\right) \tilde{C}_{2\left(\mathrm{ES}^{2}\right)}\right. \\
& \left.+\tilde{C}_{1\left(\mathrm{ES}^{2}\right)}\left(\frac{3 \nu^{3}}{2}+\frac{15}{2} \tilde{C}_{2\left(\mathrm{ES}^{2}\right)} \nu^{2}-\frac{3 X_{2} \nu^{2}}{2}+\frac{15 \nu^{2}}{2}\right)\right] \\
& +\chi_{1}^{4}\left[-\frac{3 \nu^{4}}{4}+3 \nu^{3}+\left(3 \nu^{3}-3 \nu^{2}\right) X_{1}+\left(-\frac{3 \nu^{3}}{2}+\frac{9 \nu^{2}}{2}-\frac{3 \nu}{2}+\left(\frac{9 \nu^{2}}{2}-6 \nu+\frac{3}{2}\right) X_{1}\right) \tilde{C}_{1\left(\mathrm{ES}^{2}\right)}\right. \\
& \left.+\left(-\frac{3 \nu^{2}}{4}+\frac{3 \nu}{4}+\left(\frac{3 \nu}{2}-\frac{3}{4}\right) X_{1}\right) \tilde{C}_{1\left(\mathrm{ES}^{4}\right)}\right] \\
& +\frac{1}{2}\left(\chi_{1} \cdot \chi_{2}\right)^{2}\left[-3 \nu^{4}+3 \nu^{3}+\left(6 \nu^{3}-6 \nu^{2}\right) X_{2}+\left(3 \nu^{2}-3 \nu X_{2}\right) \tilde{C}_{2\left(\mathrm{BS}^{3}\right)}+\left(3 \nu^{2} X_{2}-3 \nu^{3}\right) \tilde{C}_{2\left(\mathrm{ES}^{2}\right)}\right] \\
& +\frac{1}{2} \chi_{1}^{2} \chi_{2}^{2}\left[18 \nu^{4}-\frac{55 \nu^{3}}{2}+\left(-2 \nu^{4}-47 \nu^{3}+\frac{85 \nu^{2}}{2}\right) X_{2}+\left(15 \nu X_{2}-15 \nu^{2}\right) \tilde{C}_{2\left(\mathrm{BS}^{3}\right)}\right. \\
& \left.+\left(9 \nu^{3}+\left(-6 \nu^{3}-9 \nu^{2}\right) X_{2}\right) \tilde{C}_{2\left(\mathrm{ES}^{2}\right)}\right] \\
& +\chi_{1} \cdot \chi_{2} \chi_{1}^{2}\left[-\frac{3 \nu^{4}}{2}+3 \nu^{3}-\frac{3 \nu^{2}}{2}+\left(-\frac{3 \nu^{3}}{2}+\frac{3 X_{1} \nu^{2}}{2}-\frac{3 \nu^{2}}{2}\right) \tilde{C}_{2\left(\mathrm{ES}^{2}\right)}\right. \\
& \left.\left.+\tilde{C}_{1\left(\mathrm{ES}^{2}\right)}\left(-\frac{3 \nu^{3}}{2}-\frac{3}{2} \tilde{C}_{2\left(\mathrm{ES}^{2}\right)} \nu^{2}-\frac{3 X_{1} \nu^{2}}{2}\right)\right]\right\}+1 \leftrightarrow 2,
\end{aligned}
$$




$$
\begin{aligned}
Q^{S^{4}}= & \frac{\hat{p}_{r}^{2}}{\hat{r}^{4}}\left\{\chi_{2}^{2}\left(\boldsymbol{n} \cdot \chi_{1}\right)^{2}\left(3 \nu^{4}-6 \nu^{4} X_{2}\right)+\chi_{1} \cdot \chi_{2}\left(\boldsymbol{n} \cdot \chi_{1}\right)^{2}\left[9 \nu^{4}-3 \nu^{3}+\left(6 \nu^{4}-12 \nu^{3}+3 \nu^{2}\right) X_{1}\right]\right. \\
& \left.+\chi_{1}^{2} \boldsymbol{n} \cdot \chi_{1} \boldsymbol{n} \cdot \chi_{2}\left[-9 \nu^{4}+3 \nu^{3}+\left(-6 \nu^{4}+12 \nu^{3}-3 \nu^{2}\right) X_{1}\right]\right\} \\
& +\frac{\hat{p}_{r}}{\hat{r}^{4}}\left\{\hat { \boldsymbol { p } } \cdot \chi _ { 1 } \left[\left(\boldsymbol{n} \cdot \chi_{2}\right)^{3}\left(-12 \nu^{4}+4 \nu^{3}+\left(-8 \nu^{4}+16 \nu^{3}-4 \nu^{2}\right) X_{2}\right)+\boldsymbol{n} \cdot \chi_{1} \chi_{2}^{2}\left(8 \nu^{4} X_{2}-4 \nu^{4}\right)\right.\right. \\
& +\chi_{2}^{2} \boldsymbol{n} \cdot \chi_{2}\left(12 \nu^{4}-4 \nu^{3}+\left(8 \nu^{4}-16 \nu^{3}+4 \nu^{2}\right) X_{2}\right)+\boldsymbol{n} \cdot \chi_{1}\left(\boldsymbol{n} \cdot \chi_{2}\right)^{2}\left(8 \nu^{4}-16 \nu^{4} X_{2}\right) \\
& +\left(\boldsymbol{n} \cdot \chi_{1}\right)^{2} \boldsymbol{n} \cdot \chi_{2}\left(20 \nu^{4}-20 \nu^{3}+4 \nu^{2}+\left(-8 \nu^{4}+16 \nu^{3}-4 \nu^{2}\right) X_{2}\right) \\
& \left.\left.+\boldsymbol{n} \cdot \chi_{1} \chi_{1} \cdot \chi_{2}\left(-20 \nu^{4}+20 \nu^{3}-4 \nu^{2}+\left(8 \nu^{4}-16 \nu^{3}+4 \nu^{2}\right) X_{2}\right)\right]\right\}+1 \leftrightarrow 2 .
\end{aligned}
$$

[1] B. P. Abbott et al. (Virgo and LIGO Scientific Collaborations), Observation of Gravitational Waves from a Binary Black Hole Merger, Phys. Rev. Lett. 116, 061102 (2016).

[2] B. P. Abbott et al. (Virgo and LIGO Scientific Collaborations), Binary Black Hole Mergers in the First Advanced LIGO Observing Run, Phys. Rev. X 6, 041015 (2016).

[3] B. Abbott et al. (Virgo and LIGO Scientific Collaborations), GW170817: Observation of Gravitational Waves from a Binary Neutron Star Inspiral, Phys. Rev. Lett. 119, 161101 (2017).

[4] B. P. Abbott et al. (LIGO Scientific and Virgo Collaborations), GWTC-1: A Gravitational-Wave Transient Catalog of Compact Binary Mergers Observed by LIGO and Virgo during the First and Second Observing Runs, Phys. Rev. X 9, 031040 (2019).

[5] J. Aasi et al. (LIGO Scientific Collaboration), Advanced LIGO, Classical Quantum Gravity 32, 074001 (2015).

[6] F. Acernese et al. (Virgo Collaboration), Advanced Virgo: A second-generation interferometric gravitational wave detector, Classical Quantum Gravity 32, 024001 (2015).

[7] Y. Aso, Y. Michimura, K. Somiya, M. Ando, O. Miyakawa, T. Sekiguchi, D. Tatsumi, and H. Yamamoto (KAGRA Collaboration), Interferometer design of the KAGRA gravitational wave detector, Phys. Rev. D 88, 043007 (2013).

[8] B. Iyer et al. (LIGO Collaboration), LIGO-India, Proposal of the consortium for Indian initiative in gravitational-wave observations, LIGO Document M1100296-v2, 2011.

[9] A. Buonanno and T. Damour, Effective one-body approach to general relativistic two-body dynamics, Phys. Rev. D 59, 084006 (1999).

[10] A. Buonanno and T. Damour, Transition from inspiral to plunge in binary black hole coalescences, Phys. Rev. D 62, 064015 (2000).

[11] A. Buonanno, Y. Chen, and T. Damour, Transition from inspiral to plunge in precessing binaries of spinning black holes, Phys. Rev. D 74, 104005 (2006).

[12] T. Damour, Coalescence of two spinning black holes: An effective one-body approach, Phys. Rev. D 64, 124013 (2001).
[13] T. Damour, P. Jaranowski, and G. Schäfer, Effective one body approach to the dynamics of two spinning black holes with next-to-leading order spin-orbit coupling, Phys. Rev. D 78, 024009 (2008).

[14] A. Nagar, Effective one body Hamiltonian of two spinning black-holes with next-to-next-to-leading order spin-orbit coupling, Phys. Rev. D 84, 084028 (2011); Erratum, Phys. Rev. D 88, 089901 (2013).

[15] S. Balmelli and P. Jetzer, Effective-one-body Hamiltonian with next-to-leading order spin-spin coupling for two nonprecessing black holes with aligned spins, Phys. Rev. D 87, 124036 (2013); Erratum, Phys. Rev. D 90, 089905 (2014).

[16] S. Balmelli and P. Jetzer, Effective-one-body Hamiltonian with next-to-leading order spin-spin coupling, Phys. Rev. D 91, 064011 (2015).

[17] S. Balmelli and T. Damour, New effective-one-body Hamiltonian with next-to-leading order spin-spin coupling, Phys. Rev. D 92, 124022 (2015).

[18] Y. Pan, A. Buonanno, L. T. Buchman, T. Chu, L. E. Kidder, H. P. Pfeiffer, and M. A. Scheel, Effective-one-body waveforms calibrated to numerical relativity simulations: Coalescence of non-precessing, spinning, equal-mass black holes, Phys. Rev. D 81, 084041 (2010).

[19] T. Damour and A. Nagar, New effective-one-body description of coalescing nonprecessing spinning black-hole binaries, Phys. Rev. D 90, 044018 (2014).

[20] A. Nagar, T. Damour, C. Reisswig, and D. Pollney, Energetics and phasing of nonprecessing spinning coalescing black hole binaries, Phys. Rev. D 93, 044046 (2016).

[21] A. Nagar, G. Riemenschneider, and G. Pratten, Impact of numerical relativity information on effective-one-body waveform models, Phys. Rev. D 96, 084045 (2017).

[22] S. Akcay, S. Bernuzzi, F. Messina, A. Nagar, N. Ortiz, and P. Rettegno, Effective-one-body multipolar waveform for tidally interacting binary neutron stars up to merger, Phys. Rev. D 99, 044051 (2019).

[23] A. Nagar et al., Time-domain effective-one-body gravitational waveforms for coalescing compact binaries with nonprecessing spins, tides, and self-spin effects, Phys. Rev. D 98, 104052 (2018). 
[24] A. Nagar and P. Rettegno, Efficient effective one body time-domain gravitational waveforms, Phys. Rev. D 99, 021501 (2019).

[25] E. Barausse, E. Racine, and A. Buonanno, Hamiltonian of a spinning test-particle in curved spacetime, Phys. Rev. D 80, 104025 (2009); Erratum, Phys. Rev. D 85, 069904 (2012).

[26] J. Vines, D. Kunst, J. Steinhoff, and T. Hinderer, Canonical Hamiltonian for an extended test body in curved spacetime: To quadratic order in spin, Phys. Rev. D 93, 103008 (2016).

[27] E. Barausse and A. Buonanno, An improved effective-onebody Hamiltonian for spinning black-hole binaries, Phys. Rev. D 81, 084024 (2010).

[28] E. Barausse and A. Buonanno, Extending the effectiveone-body Hamiltonian of black-hole binaries to include next-to-next-to-leading spin-orbit couplings, Phys. Rev. D 84, 104027 (2011).

[29] T. Hinderer et al., Effects of Neutron-Star Dynamic Tides on Gravitational Waveforms within the Effective-OneBody Approach, Phys. Rev. Lett. 116, 181101 (2016).

[30] J. Steinhoff, T. Hinderer, A. Buonanno, and A. Taracchini, Dynamical tides in general relativity: Effective action and effective-one-body Hamiltonian, Phys. Rev. D 94, 104028 (2016).

[31] N. Yunes, A. Buonanno, S. A. Hughes, Y. Pan, E. Barausse, M. C. Miller, and W. Throwe, Extreme massratio inspirals in the effective-one-body approach: Quasicircular, equatorial orbits around a spinning black hole, Phys. Rev. D 83, 044044 (2011); Erratum, Phys. Rev. D 88, 109904 (2013).

[32] T. Hinderer et al., Periastron advance in spinning black hole binaries: Comparing effective-one-body and numerical relativity, Phys. Rev. D 88, 084005 (2013).

[33] A. Taracchini, Y. Pan, A. Buonanno, E. Barausse, M. Boyle, T. Chu, G. Lovelace, H. P. Pfeiffer, and M. A. Scheel, Prototype effective-one-body model for nonprecessing spinning inspiral-merger-ringdown waveforms, Phys. Rev. D 86, 024011 (2012).

[34] A. Taracchini et al., Effective-one-body model for blackhole binaries with generic mass ratios and spins, Phys. Rev. D 89, 061502 (2014).

[35] Y. Pan, A. Buonanno, A. Taracchini, L. E. Kidder, A. H. Mroué, H. P. Pfeiffer, M. A. Scheel, and B. Szilágyi, Inspiral-merger-ringdown waveforms of spinning, precessing black-hole binaries in the effective-one-body formalism, Phys. Rev. D 89, 084006 (2014).

[36] A. Bohé et al., Improved effective-one-body model of spinning, nonprecessing binary black holes for the era of gravitational-wave astrophysics with advanced detectors, Phys. Rev. D 95, 044028 (2017).

[37] S. Babak, A. Taracchini, and A. Buonanno, Validating the effective-one-body model of spinning, precessing binary black holes against numerical relativity, Phys. Rev. D 95, 024010 (2017).

[38] R. Cotesta, A. Buonanno, A. Bohé, A. Taracchini, I. Hinder, and S. Ossokine, Enriching the symphony of gravitational waves from binary black holes by tuning higher harmonics, Phys. Rev. D 98, 084028 (2018).

[39] T. Damour, Gravitational self force in a Schwarzschild background and the effective one body formalism, Phys. Rev. D 81, 024017 (2010).
[40] S. Akcay, L. Barack, T. Damour, and N. Sago, Gravitational self-force and the effective-one-body formalism between the innermost stable circular orbit and the light ring, Phys. Rev. D 86, 104041 (2012).

[41] E. Barausse, A. Buonanno, and A. Le Tiec, The complete non-spinning effective-one-body metric at linear order in the mass ratio, Phys. Rev. D 85, 064010 (2012).

[42] A. Antonelli, M. van de Meent, A. Buonanno, J. Steinhoff, and J. Vines, Quasicircular inspirals and plunges from nonspinning effective-one-body Hamiltonians with gravitational self-force information, Phys. Rev. D 101, 024024 (2020).

[43] T. Damour, Gravitational scattering, post-Minkowskian approximation, and effective one-body theory, Phys. Rev. D 94, 104015 (2016).

[44] T. Damour, High-energy gravitational scattering and the general relativistic two-body problem, Phys. Rev. D 97, 044038 (2018).

[45] A. Antonelli, A. Buonanno, J. Steinhoff, M. van de Meent, and J. Vines, Energetics of two-body Hamiltonians in postMinkowskian gravity, Phys. Rev. D 99, 104004 (2019).

[46] P. Rettegno, F. Martinetti, A. Nagar, D. Bini, G. Riemenschneider, and T. Damour, Comparing effective one body Hamiltonians for spin-aligned coalescing binaries, arXiv:1911.10818.

[47] A. Nagar, F. Messina, P. Rettegno, D. Bini, T. Damour, A. Geralico, S. Akcay, and S. Bernuzzi, Nonlinear-in-spin effects in effective-one-body waveform models of spinaligned, inspiralling, neutron star binaries, Phys. Rev. D 99, 044007 (2019).

[48] T. Damour, B. R. Iyer, and A. Nagar, Improved resummation of post-Newtonian multipolar waveforms from circularized compact binaries, Phys. Rev. D 79, 064004 (2009).

[49] M. Levi and J. Steinhoff, Complete conservative dynamics for inspiralling compact binaries with spins at fourth postNewtonian order, arXiv:1607.04252.

[50] M. Mathisson, Neue mechanik materieller systemes, Acta Phys. Pol. 6, 163 (1937).

[51] M. Mathisson, Republication of: New mechanics of material systems, Gen. Relativ. Gravit. 42, 1011 (2010).

[52] A. Papapetrou, Spinning test particles in general relativity. 1. Proc. R. Soc. A 209, 248 (1951).

[53] E. Corinaldesi and A. Papapetrou, Spinning test particles in general relativity. 2. Proc. R. Soc. A 209, 259 (1951).

[54] W. G. Dixon, Extended bodies in general relativity: Their description and motion, in Isolated Gravitating Systems in General Relativity, edited by J. Ehlers (North Holland Publishing Co., Amsterdam, Netherlands, 1979), pp. 156-219.

[55] T. Damour, P. Jaranowski, and G. Schäfer, On the determination of the last stable orbit for circular general relativistic binaries at the third post-Newtonian approximation, Phys. Rev. D 62, 084011 (2000).

[56] D. Bini, T. Damour, and A. Geralico, Spin-dependent twobody interactions from gravitational self-force computations, Phys. Rev. D 92, 124058 (2015); Erratum, Phys. Rev. D 93, 109902 (2016).

[57] M. Levi and J. Steinhoff, Spinning gravitating objects in the effective field theory in the post-Newtonian scheme, J. High Energy Phys. 09 (2015) 219. 
[58] M. Levi and J. Steinhoff, Next-to-next-to-leading order gravitational spin-squared potential via the effective field theory for spinning objects in the post-Newtonian scheme, J. Cosmol. Astropart. Phys. 01 (2016) 008.

[59] W. Tulczyjew, Equations of motion of rotating bodies in general relativity theory, Acta Phys. Pol. 18, 37 (1959); Erratum, Acta Phys. Pol. 18, 534 (1959).

[60] B. M. Barker and R.F. O'Connell, Derivation of the equations of motion of a gyroscope from the quantum theory of gravitation, Phys. Rev. D 2, 1428 (1970).

[61] B. M. Barker and R. F. O'Connell, Gravitational two-body problem with arbitrary masses, spins, and quadrupole moments, Phys. Rev. D 12, 329 (1975).

[62] P. D. D'Eath, Interaction of two black holes in the slowmotion limit, Phys. Rev. D 12, 2183 (1975).

[63] B. M. Barker and R.F. O'Connell, The gravitational interaction: Spin, rotation, and quantum effects-A review, Gen. Relativ. Gravit. 11, 149 (1979).

[64] T. Damour, Probléme des deux corps et freinage de rayonnement en relativité générale, C. R. Acad. Sci. Paris Sér. II 294, 1355 (1982).

[65] K. S. Thorne and J. B. Hartle, Laws of motion and precession for black holes and other bodies, Phys. Rev. D 31, 1815 (1985).

[66] T. Damour and G. Schäfer, Higher order relativistic Periastron advances and binary pulsars, Nuovo Cimento B 101, 127 (1988).

[67] H. Tagoshi, A. Ohashi, and B. J. Owen, Gravitational field and equations of motion of spinning compact binaries to 2.5 post-Newtonian order, Phys. Rev. D 63, 044006 (2001).

[68] G. Faye, L. Blanchet, and A. Buonanno, Higher-order spin effects in the dynamics of compact binaries. I. Equations of motion, Phys. Rev. D 74, 104033 (2006).

[69] T. Damour, P. Jaranowski, and G. Schäfer, Hamiltonian of two spinning compact bodies with next-to-leading order gravitational spin-orbit coupling, Phys. Rev. D 77, 064032 (2008).

[70] J. Steinhoff, G. Schäfer, and S. Hergt, ADM canonical formalism for gravitating spinning objects, Phys. Rev. D 77, 104018 (2008).

[71] D. L. Perrodin, Subleading spin-orbit correction to the Newtonian potential in effective field theory formalism, in Proceedings of the 12th Marcel Grossmann Meeting on General Relativity (World Scientific, Singapore, 2011), pp. 725-727.

[72] R. A. Porto, Next to leading order spin-orbit effects in the motion of inspiralling compact binaries, Classical Quantum Gravity 27, 205001 (2010).

[73] M. Levi, Next to leading order gravitational spin-orbit coupling in an effective field theory approach, Phys. Rev. D 82, 104004 (2010).

[74] J. Hartung and J. Steinhoff, Next-to-next-to-leading order post-Newtonian spin-orbit Hamiltonian for self-gravitating binaries, Ann. Phys. (Amsterdam) 523, 783 (2011).

[75] J. Hartung, J. Steinhoff, and G. Schäfer, Next-to-nextto-leading order post-Newtonian linear-in-spin binary Hamiltonians, Ann. Phys. (Amsterdam) 525, 359 (2013).

[76] M. Levi and J. Steinhoff, Next-to-next-to-leading order gravitational spin-orbit coupling via the effective field theory for spinning objects in the post-Newtonian scheme, J. Cosmol. Astropart. Phys. 01 (2016) 011.
[77] S. Marsat, A. Bohe, G. Faye, and L. Blanchet, Next-tonext-to-leading order spin-orbit effects in the equations of motion of compact binary systems, Classical Quantum Gravity 30, 055007 (2013).

[78] A. Bohe, S. Marsat, G. Faye, and L. Blanchet, Next-tonext-to-leading order spin-orbit effects in the near-zone metric and precession equations of compact binaries, Classical Quantum Gravity 30, 075017 (2013).

[79] E. Poisson, Gravitational waves from inspiraling compact binaries: The quadrupole moment term, Phys. Rev. D 57, 5287 (1998).

[80] J. Steinhoff, S. Hergt, and G. Schäfer, On the next-toleading order gravitational spin(1)-spin(2) dynamics, Phys. Rev. D 77, 081501 (2008).

[81] R. A. Porto and I. Z. Rothstein, Calculation of the First Nonlinear Contribution to the General-Relativistic SpinSpin Interaction for Binary Systems, Phys. Rev. Lett. 97, 021101 (2006).

[82] R. A. Porto and I. Z. Rothstein, Spin(1)spin(2) effects in the motion of inspiralling compact binaries at third order in the post-Newtonian expansion, Phys. Rev. D 78, 044012 (2008); Erratum, Phys. Rev. D 81, 029904 (2010).

[83] M. Levi, Next to leading order gravitational spin1-spin2 coupling with Kaluza-Klein reduction, Phys. Rev. D 82, 064029 (2010).

[84] R. A. Porto and I. Z. Rothstein, Next to leading order spin(1)spin(1) effects in the motion of inspiralling compact binaries, Phys. Rev. D 78, 044013 (2008); Erratum, Phys. Rev. D 81, 029905 (2010).

[85] J. Steinhoff, S. Hergt, and G. Schäfer, Spin-squared Hamiltonian of next-to-leading order gravitational interaction, Phys. Rev. D 78, 101503 (2008).

[86] S. Hergt, J. Steinhoff, and G. Schäfer, Reduced Hamiltonian for next-to-leading order spin-squared dynamics of general compact binaries, Classical Quantum Gravity 27, 135007 (2010).

[87] S. Hergt, J. Steinhoff, and G. Schäfer, Elimination of the spin supplementary condition in the effective field theory approach to the post-Newtonian approximation, Ann. Phys. (Amsterdam) 327, 1494 (2012).

[88] A. Bohé, G. Faye, S. Marsat, and E. K. Porter, Quadratic-in-spin effects in the orbital dynamics and gravitational-wave energy flux of compact binaries at the 3PN order, Classical Quantum Gravity 32, 195010 (2015).

[89] J. Hartung and J. Steinhoff, Next-to-next-to-leading order post-Newtonian spin(1)-spin(2) Hamiltonian for selfgravitating binaries, Ann. Phys. (Amsterdam) 523, 919 (2011).

[90] M. Levi, Binary dynamics from spin1-spin2 coupling at fourth post-Newtonian order, Phys. Rev. D 85, 064043 (2012).

[91] M. Levi and J. Steinhoff, Equivalence of ADM Hamiltonian and effective field theory approaches at next-to-next-toleading order spin1-spin2 coupling of binary inspirals, J. Cosmol. Astropart. Phys. 12 (2014) 003.

[92] S. Hergt and G. Schäfer, Higher-order-in-spin interaction Hamiltonians for binary black holes from source terms of Kerr geometry in approximate ADM coordinates, Phys. Rev. D 77, 104001 (2008). 
[93] S. Hergt and G. Schäfer, Higher-order-in-spin interaction Hamiltonians for binary black holes from Poincare invariance, Phys. Rev. D 78, 124004 (2008).

[94] M. Levi and J. Steinhoff, Leading order finite size effects with spins for inspiralling compact binaries, J. High Energy Phys. 06 (2015) 059.

[95] S. Marsat, Cubic order spin effects in the dynamics and gravitational wave energy flux of compact object binaries, Classical Quantum Gravity 32, 085008 (2015).

[96] V. Vaidya, Gravitational spin Hamiltonians from the S matrix, Phys. Rev. D 91, 024017 (2015).

[97] J. B. Gilmore and A. Ross, Effective field theory calculation of second post-Newtonian binary dynamics, Phys. Rev. D 78, 124021 (2008).

[98] T. Damour, P. Jaranowski, and G. Schäfer, Nonlocal-intime action for the fourth post-Newtonian conservative dynamics of two-body systems, Phys. Rev. D 89, 064058 (2014).

[99] L. Bernard, L. Blanchet, A. Bohé, G. Faye, and S. Marsat, Energy and periastron advance of compact binaries on circular orbits at the fourth post-Newtonian order, Phys. Rev. D 95, 044026 (2017).

[100] S. Foffa and R. Sturani, Conservative dynamics of binary systems to fourth post-Newtonian order in the EFT approach I: Regularized Lagrangian, Phys. Rev. D 100, 024047 (2019).

[101] J. Blümlein, A. Maier, P. Marquard, and G. Schäfer, Fourth post-Newtonian Hamiltonian dynamics of two-body systems from an effective field theory approach, arXiv: 2003.01692.
[102] S. Foffa, P. Mastrolia, R. Sturani, C. Sturm, and W. J. Torres Bobadilla, Static Two-Body Potential at Fifth PostNewtonian Order, Phys. Rev. Lett. 122, 241605 (2019).

[103] J. Blümlein, A. Maier, and P. Marquard, Five-loop static contribution to the gravitational interaction potential of two point masses, Phys. Lett. B 800, 135100 (2020).

[104] D. Bini, T. Damour, and A. Geralico, Novel Approach to Binary Dynamics: Application to the Fifth Post-Newtonian Level, Phys. Rev. Lett. 123, 231104 (2019).

[105] See Supplemental Material at http://link.aps.org/ supplemental/10.1103/PhysRevD.101.104034 were provided, in the form of Mathematica files, for the expressions for the SEOB Hamiltonians, and the potentials given in the paper.

[106] J. Vines and J. Steinhoff, Spin-multipole effects in binary black holes and the test-body limit, Phys. Rev. D 97, 064010 (2018).

[107] T. Damour, P. Jaranowski, and G. Schäfer, Fourth postNewtonian effective one-body dynamics, Phys. Rev. D 91, 084024 (2015).

[108] https://data.black-holes.org/waveforms.

[109] S. Ossokine, T. Dietrich, E. Foley, R. Katebi, and G. Lovelace, Assessing the energetics of spinning binary black hole systems, Phys. Rev. D 98, 104057 (2018).

[110] T. Dietrich, S. Bernuzzi, M. Ujevic, and W. Tichy, Gravitational waves and mass ejecta from binary neutron star mergers: Effect of the stars' rotation, Phys. Rev. D 95, 044045 (2017). 